\title{
Cultural Resilience \\ Architecture in Post-Disaster Planning
}

by

James B. Robinson

A thesis submitted to the Faculty of Graduate and Postdoctoral Affairs In partial fulfillment of the requirements for the degree of

Master of Architecture

M.Arch. Professional

Carleton University

Ottawa, Ontario

(C) 2011

James B. Robinson 
Library and Archives

Canada

Published Heritage

Branch

395 Wellington Street

Ottawa ON K1A ON4

Canada
Bibliothèque et

Archives Canada

Direction du

Patrimoine de l'édition

395 , rue Wellington

Ottawa ON K1A ON4

Canada
Your file Votre référence

ISBN: 978-0-494-81595-3

Our file Notre référence

ISBN: 978-0-494-81595-3
NOTICE:

The author has granted a nonexclusive license allowing Library and Archives Canada to reproduce, publish, archive, preserve, conserve, communicate to the public by telecommunication or on the Internet, loan, distribute and sell theses worldwide, for commercial or noncommercial purposes, in microform, paper, electronic and/or any other formats.

The author retains copyright ownership and moral rights in this thesis. Neither the thesis nor substantial extracts from it may be printed or otherwise reproduced without the author's permission.
AVIS:

L'auteur a accordé une licence non exclusive permettant à la Bibliothèque et Archives Canada de reproduire, publier, archiver, sauvegarder, conserver, transmettre au public par télécommunication ou par l'Internet, prêter, distribuer et vendre des thèses partout dans le monde, à des fins commerciales ou autres, sur support microforme, papier, électronique et/ou autres formats.

L'auteur conserve la propriété du droit d'auteur et des droits moraux qui protège cette thèse. $\mathrm{Ni}$ la thèse ni des extraits substantiels de celle-ci ne doivent être imprimés ou autrement reproduits sans son autorisation.
In compliance with the Canadian Privacy Act some supporting forms may have been removed from this thesis.

While these forms may be included in the document page count, their removal does not represent any loss of content from the thesis.
Conformément à la loi canadienne sur la protection de la vie privée, quelques formulaires secondaires ont été enlevés de cette thèse.

Bien que ces formulaires aient inclus dans la pagination, il n'y aura aucun contenu manquant. 


\section{Abstract}

If a post-disaster rebuilding plan is to succeed in the long term, it needs to embody a collective of ideas that translate culture into built form. A culture is a complex puzzle of fragmented, localized knowledge. When it breaks down, a rebuilding initiative can attempt to piece it back together. Since rebuilding is specific to context, it is crucial to consider democratically collected local knowledge that is accurately translated so cultural beliefs are embraced, historical knowledge preserved, and long-term subsistence occur.

This research paper has three primary objectives: to identify the relationship between long-term strategic planning and the importance of culturally specific community rebuilding; to examine successful and unsuccessful community rebuilding case studies, noting the role played by local knowledge and collaborative design; and to determine potential for a theory-based formulaic response to long-term redevelopment that could be applied either in general or to similar rebuilding scenarios.

Finally, a hypothesis-driven project of architecture analyzes the cultural resilience of a disaster-struck community in Léogâne, Haiti. This case study both ties to the paper's main objectives and serves as validation to confirm research suppositions that see architecture as the catalyst for a culturally sensitive, longterm rebuilding strategy. 


\section{Acknowledgements}

I would like to dedicate this thesis to the local participants of the Rebuilding of Léogâne

Haiti Design Workshop 2011. Realizing you have a voice is a powerful thing. I wish you and your town all the best in rebuilding for the future.

I would also like to offer my regards to those of my friends and family who have supported me in any respect during the completion of my studies; I am truly blessed to have such meaningful people in my life. 


\section{Preface}

When referring to 'post-disaster,' this research focuses on 'the calm after the storm', the period of time in which immediate aid has been provided and long-term subsistence is ready to be explored.

These issues are not as transparent as the need to provide first aid, food, water, and shelter; these issues look to a more long-term solution for restoring communities back to how they once were. These issues address the daily interconnectivity of a community's social landscape, speaking to who and what a specific civilization stands for through a cultural perspective.

Natural disasters are an inevitable fact of life, one which global civilizations have dealt with since time immemorial. One might assume that since communities have had to deal with natural disasters for hundreds of years, some sort of formula or disaster protocol relating to post-disaster mitigation would be well entrenched. In fact, it appears to be quite the contrary-and with good reason. No two civilizations are exactly the same; in terms of economics, demographics, geography or culture. A disaster recovery methodology applied successfully to one specific context is not necessarily the correct approach for another disaster-struck community. Logic therefore would dictate that in order to formulate successful rebuilding strategy, localized knowledge would be key in regenerating its way of life. As well, local citizens, as the beneficiaries and users of rebuilding initiatives, should naturally play pivotal roles in any new design and construction.

Understanding the need to include local knowledge at the crux for successful community rebuilding. The next challenge is determining how 
outsiders coming in to offer aid can democratically—and collaboratively-collect credible information from a foreign population of people. There are many case examples of successful participatory techniques that involve local people in all stages of the design through to construction in post-disaster initiatives. Is there a specific technique of participatory collaboration that is more useful than the next? Perhaps success lies in a hybridized participatory method taken from several case examples that have worked well in the past. Or maybe the correct approach is a delicate mixture of research-driven outside consultation mixed with localized intervention to verify and impel the project. What is certain is the need to trust locally gathered information and ensure that democratically collected information is properly organized and not contributing to existing post-disaster squalor.

Utilizing a framework rooted in localized knowledge and participatory design, the 2010 Haitian earthquake provides an ongoing case study in understanding the nuances of long-term community design and reconstruction in terms of determining a best approach to a real-world scenario.

Certainly the world will continue to monitor rebuilding efforts as they unfold in this devastatingly poor country, which captured global attention on January 12 , 2010 when it was shook by a magnitude 7.3 earthquake. This natural disaster left a catastrophic trail of destruction, with over 1.5 million people directly affected (approximately $15 \%$ of Haiti's population) and an estimated 300,000 dead and the same amount injured. ${ }^{1}$ An unfair blow to a country the already exists in abject

\footnotetext{
${ }^{1}$ Haitian Government. Action Plan for National Recovery and Development in Haiti (March 2010) p.7.
} 
poverty: the United Nations Human Development Index 2010 confirms that Haiti remains among 25 of the world's poorest and most underdeveloped countries. ${ }^{2}$

The approach I will take in this research project will be similar to that of an outside design and planning consultant. This will allow me to evaluate my findings and formulate a hypothesis from an unbiased perspective on all accounts. The organization of my research is as follows: laying a foundation with the importance of local knowledge in post-disaster planning; exploring community planning via collaborative design; and using these findings to derive my position for a real-world case study with architecture as the agent. My position will involve proposing a project of architecture that addresses the need for long-term subsistence goals within a developing nation in a post-disaster context.

Furthermore, in March 2011 I travelled to Haiti as an envoy with the charity Schools for Children of the World. I was part of a planning excursion team that orchestrated a master planning workshop for the town of Léogâne with participation from local government officials, social workers, school board members and design professionals, etc. The information gathered from this collaborative design exercise, along with previously accumulated positions, served to build and enhance my understandings of local knowledge and collaborative design within the realm of a post-disaster developing nation scenario.

\footnotetext{
${ }^{2}$ United Nations Development Programme. United Nations Development Report (2010) p.145.
} 


\title{
Contents
}

\author{
ii. Abstract \\ iii. Acknowledgments \\ iv. Preface
}

I. Cultural Resilience: Exploring the Concept of Local Knowledge

IV. Haiti, 2010: Historical Overview \& Rebuilding Context Explored

Figures 


\section{Cultural Resilience: Exploring The Concept of Local Knowledge}

In order to best align the direction of this research with the reader, the term 'cultural resilience' has been used to address intent. When broken down, the term 'cultural' simply refers to 'relating to a specific civilization,' and the term 'resilience' is defined as 'the power or ability to return to the original form' and 'the ability to recover readily from illness, depression and adversity.' When taken in the context of this project it translates thusly: How can a community avoid cultural erasure and rebound back from a catastrophic natural disaster? The answers exist in the research to follow, beginning with understanding the nuances of a culture via local knowledge.

"Culture is a collection of knowledge that has evolved from generation to generation as a record of what once existed. A localized understanding of place, people and social fabric is instilled into the hearts and minds of a civilization and this is what becomes the cause and effect for their way and meaning of life. This materialization of place unknowingly lives in the sub-conscience of a person's mind and is referenced when going about daily tasks. "Everyone has practical, usually tacit knowledge of their social and physical environment, a competence reflected in 'knowing how to go on' in the routine activities of everyday life, and the capacity to improvise and innovate when necessary".

It is this subconscious relationship between body and place that allows people to recall traditions and ways of life that were once their everyday norm.

\footnotetext{
${ }^{1}$ Giddens qtd. in Roy Ellen. Indigenous Environmental Knowledge and its Transformations: Critical Anthropological Perspectives (New York: Routledge, 2000).
} 
This understanding of place is usually referred to as either 'traditional' or 'indigenous' knowledge depending on the global context of the reference. The main difference is that indigenous knowledge refers to the origins of a culture that has been less tainted by innovations of modern day society. In North America, the boundary is fairly distinct whereas indigenous refers to Aboriginal people and communities less tainted by mainstream culture, and traditional streams of knowledge refer to all other information and culture being practiced. Often in older and less developed civilizations, the relationship between traditional and indigenous knowledge is more cohesive and tends to blend into the same category of just 'traditional knowledge,' as is the case for Haiti.

It is the accumulation of this traditional knowledge that informs the broader concept of vernacular and the even larger category of regionalism, which can be defined as 'the analysis of a culture over time from a macro perspective.' A successful rebuilding strategy will ultimately address regionalism by utilizing fragments of a vernacular strategy to find its place within an existing network of local design and planning. Consider the following example:

On October 8, 2005, a massive earthquake recording 7.6 magnitude on the Richter scale shook North West Pakistan \& Pakistan Administered Kashmir. This natural disaster devastated over $30,000 \mathrm{sq} / \mathrm{km}$ of mountainous northern Pakistan leaving tens of thousands of causalities and more than 3.5 million people homeless. In order to efficiently mitigate a successful reconstruction initiative, UN Habitat in association with WWF Pakistan and the Emergency Architects utilized local knowledge, materials and training to initiate a self-help housing rebuilding strategy. The methods of Taq and Dhaji 
construction were identified as earthquake resistant vernacular architecture that had been successfully used in this seismically active region of Pakistan for hundreds of years. $^{2}$

This strategy of infill panel construction is successful in displacing movement through the structure of the walls leaving the house or building intact postearthquake. This age-old building technique is a prime example of a localized solution sympathetic with traditional knowledge that was used to mitigate the rebuilding process of an earthquake-stricken community.

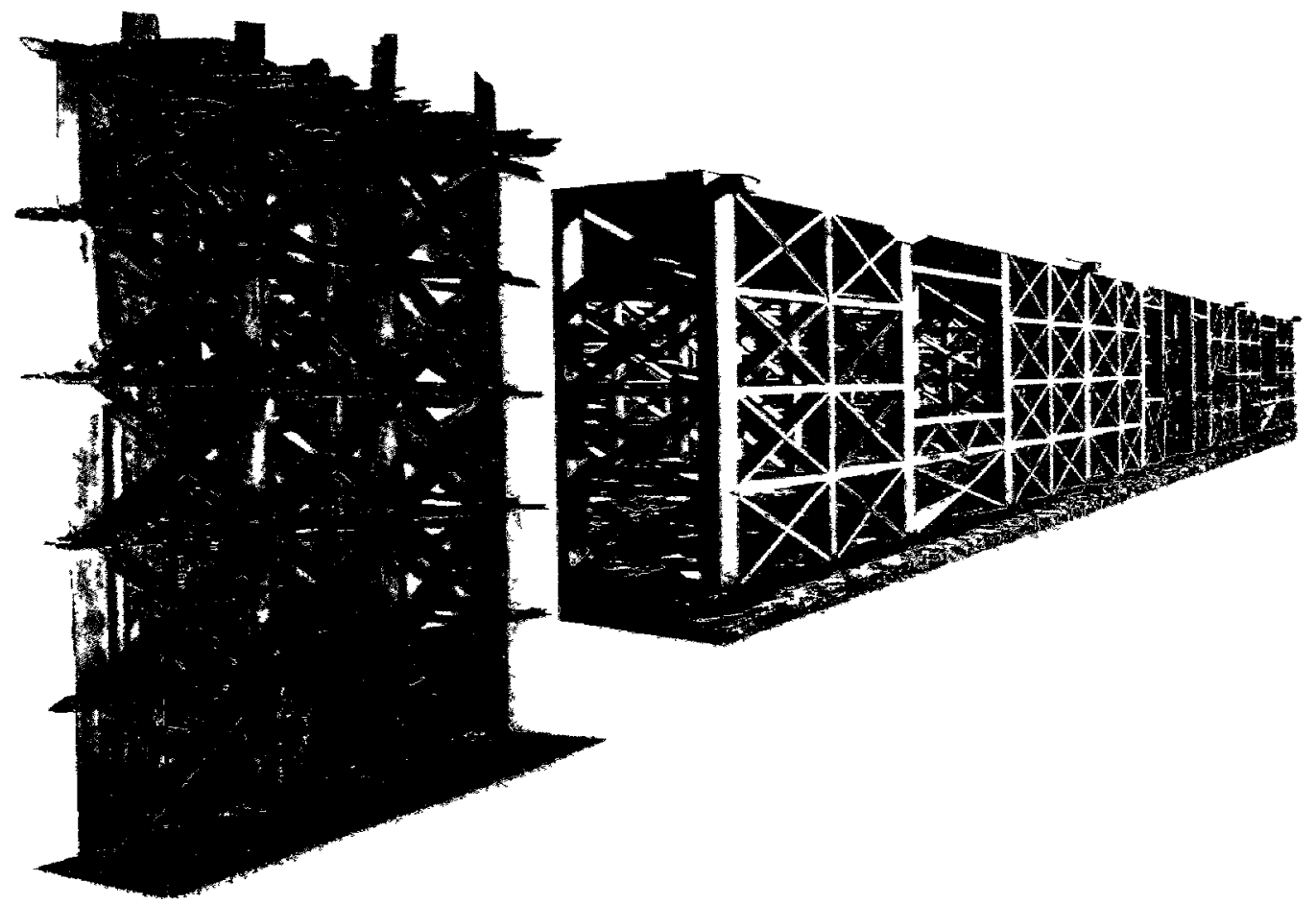

Fig. 1.1 - Taq \& Dhajji construction techniques. (Left) $18^{\text {th }}$ Century Model of the "gaiola" frame "invented" after the 1775 earthquake specifically for earthquake resistance. (Right) Dhajii house under construction in Pakistan Administered Kashmir after the 2005 earthquake". ${ }^{3}$

\footnotetext{
${ }^{2}$ UN-Habitat. Earthquake Disaster - Pakistan. United Nations Human Settlements Programme; <http://ww2.unhabitat.org/earthquake/Pakistan_earthquake.asp>. Accessed 23/09/2010.

${ }^{3}$ Randolph Langenbach. Don't Tear It Down! Preserving the Earthquake Resistant Vernacular Architecture of Kashmir (Oakland: Oinfroin Media, 2009) p.19.
} 


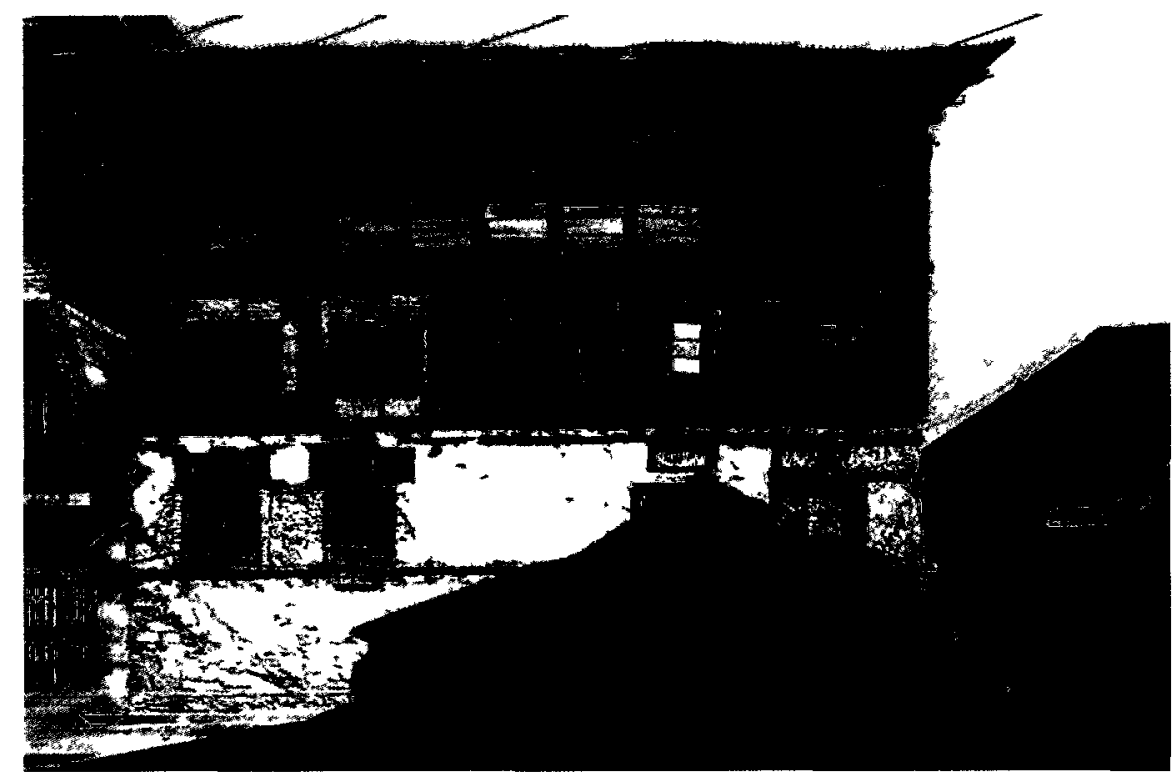

Fig. 1.2 - An example of Taq construction in Srinagar, India. The symmetrical layout of windows is characteristic of Taq and it is from this that the name of the construction is derived".

In the case of the 2005 Pakistan earthquake, an extremely effective and technically strong vernacular solution was the answer to successful reconstruction, but there will not always be such effective local solutions available. Sometimes all signs of regionalism will be completely dissipated with nothing but sparse fragments of place and local knowledge pointing to what may have once constituted the vernacular of a given community, as is often the case when disaster strikes developing nations. It is then the responsibility of everyone involved in the redesign process to translate and embrace local culture to properly rebuild with long-term sustainability in mind.

According to Coburn et al, there are three crucial factors to do with culture and community that should be used to help determine the success of any type of resettlement project: "The physical environment of the new settlement, the

\footnotetext{
${ }^{4}$ Langenbach. Don't Tear It Down! p.9.
} 
relationship to the old village and the capability of the community to develop itself'. ${ }^{5}$ The 2005 Pakistan earthquake case study is a good example of how a project can satisfy all of these crucial factors. In this instance, it just so happens that traditional methods of Taq and Dhajji Dewari construction provide the one common denominator.

The first crucial factor for successful resettlement is 'physical environment of the new settlement.' This aspect is satisfied since Pakistan's North West Frontier Province and Pakistan Administered Kashmir did not need to move geographic location. These communities have existed in unstable mountainous soil for hundreds of years, but due to their highly stable traditional construction methods of Taq and Dhajji Dewari construction, it made staying put a viable option. The second crucial factor in determining the success of a resettlement project is 'its relationship to its old village' before a disaster. In the Pakistan earthquake, for example, it was simply a matter of re-identifying their usage of traditional building techniques as 'the connection to their destroyed community' and using this in their rebuilding initiative. The final factor to determining the success of a resettlement project according to Coburn et al is accessing a community's 'capability to develop its own self.' Programs were initiated in the disaster struck area of Pakistan and Pakistan Administered Kashmir that reintroduced and honed the skills of traditional construction techniques thus increasing the capacities and likeliness of the community to develop and one day sustain its own existence.

\footnotetext{
${ }^{5}$ A. Oliver-Smith. Successes and Failures in Post-Disaster Resettlement. Disasters 15.1 (2007) p.15.
} 


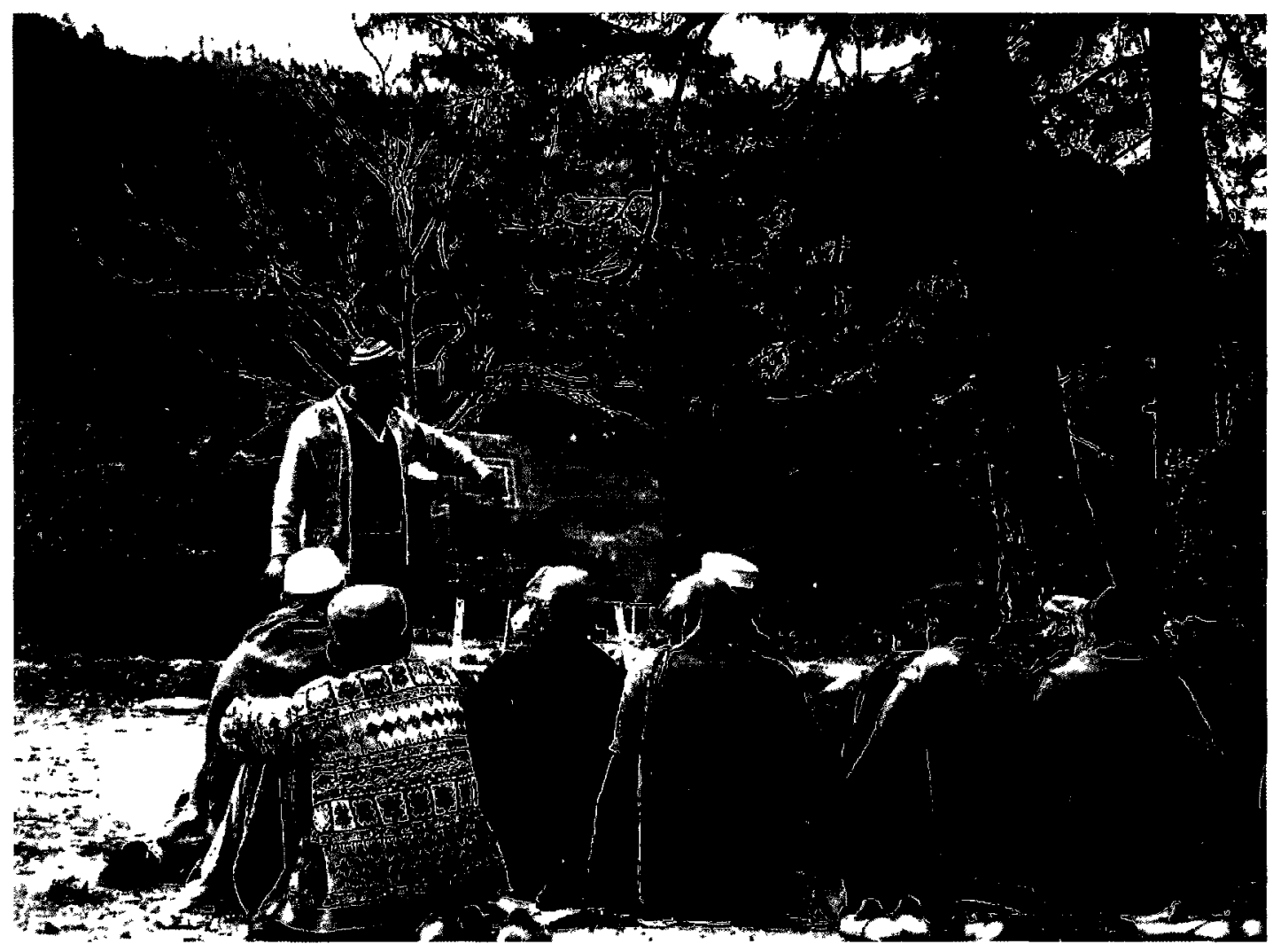

Fig. 1.3 - Dhaiji field demonstration, Pakistan. Local persons being introduced and trained in the proper techniques of Dhajii construction.

A case study from the 1992 earthquake in Flores Island, Indonesia, serves to demonstrate that without careful cultural considerations and attention to Coburn's crucial factors for resettlement, a community rebuilding project is very unlikely to succeed in the long term, as is illustrated in this example:

"On December 12, 1992 off the North coast of Flores Island, Indonesia, an earthquake hit which devastated the area effecting 30,000 buildings, out of which half were totally or partially collapsed not to mention the thousands of local people who were either misplaced, hurt or killed as result. Also many buildings along the coastline were heavily damaged due to resulting liquefaction and tsunami. 
"After the earthquake, the tsunami stricken areas such as Wuring village and a village on Babi /sland were declared dangerous for habitation. Therefore on the advice of several 'international experts' it was decided to relocate these villages to new areas, namely Nangahure and Nangahale. Subsequently in 1993, as part of their 'public services,' the armed forces built 800 houses in Nangahure and 1,000 houses in Nangahale for the earthquake victims after relocating people from Wuring village to Nangahure and those from Babi Island village to Nangahale.

"Eight years after the 1992 earthquake, many houses in Nangahure were abandoned because people moved back to their original village of Wuring. It is apparent that the population of Wuring village, which consisted of Bajo and Bugis ethnic groups originating from South Sulawesi, felt more at home in Wuring, which was established by their ancestors a few hundred years ago. It is apparent that the decision to relocate them in 1993 to Nangahure did not take the social, cultural and economic factors into account. A preliminary survey in March 2001 reveals among others the following findings:

"Nangahure was located inland approx 200 meters from the shoreline (far from the beach, because according to the experts' advice it shall be relocated inland to avoid tsunami) and the types of houses built were similar to that in a military barrack. In Wuring, the houses were built on poles so that these do not get submerged in water during high tides. These were also in line with their ways of living as fishermen who consider sea as part of their life. By building on poles, the fishermen could tie their boats along their houses during high tides. However, they could not do so in Nangahure because the barracklike houses were built on land. 
"As mentioned earlier, the population of Nangahure was not entirely from Wuring village. Besides those families, which originated from Wuring, around 500 families came from Maumere. The people from around Maumere consisted of native Flores people and most of them worked in Maumere. Most of these people were devote Catholics, while the Bugis and Bajos were Muslim and their cultures also differed. For centuries they had lived in harmony in Flores since they had their own areas/villages. In Nangahure, the government overlooked this problem and settled these two culturally and socially distinct groups in one village.

"Therefore, the natives of Wuring did not feel at home when forced to move to Nangahure in 1992. For centuries, they had retained their culture, social environment etc. and apparently had problems in totally changing their habits, environment etc.

"In order to prevent the majority of the native Wuring population from leaving Nangahure, the Government built several mosques, a fish auction building and other public facilities etc. However, it seemed that all those efforts could not stop the strong instinct and determination of the native Wuring population to return to their native village in Wuring.

After eight years, most of them had rebuilt their houses on poles and their mosque, and moved back to their old habitat in Wuring. Ironically, the situation in Wuring in 2001 practically returned to the pre-1992 earthquake condition". 6

\footnotetext{
${ }^{6}$ Teddy Boeni \& Rohit Jigyasuii. Cultural Considerations for Post Disaster Reconstruction Post Tsunami Challenges (2004) p.2-3.
} 


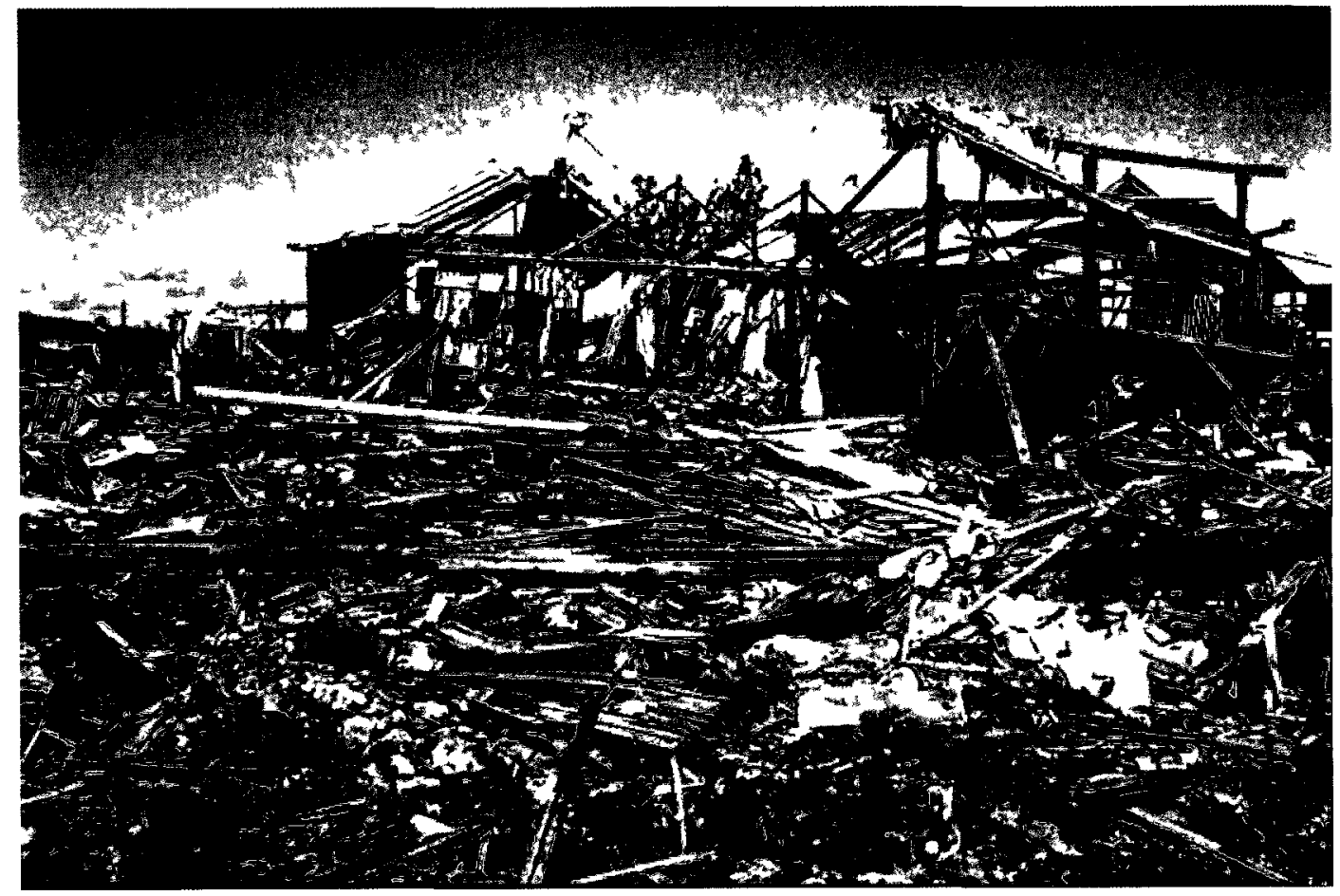

Fig. 1.4 - Post 1992 Earthquake and Tsunami at Wuhring, Flores Island, Indonesia Waves swept over this thin penınsula community devastating the community. Here the damage was not as severe as on Babi Island.

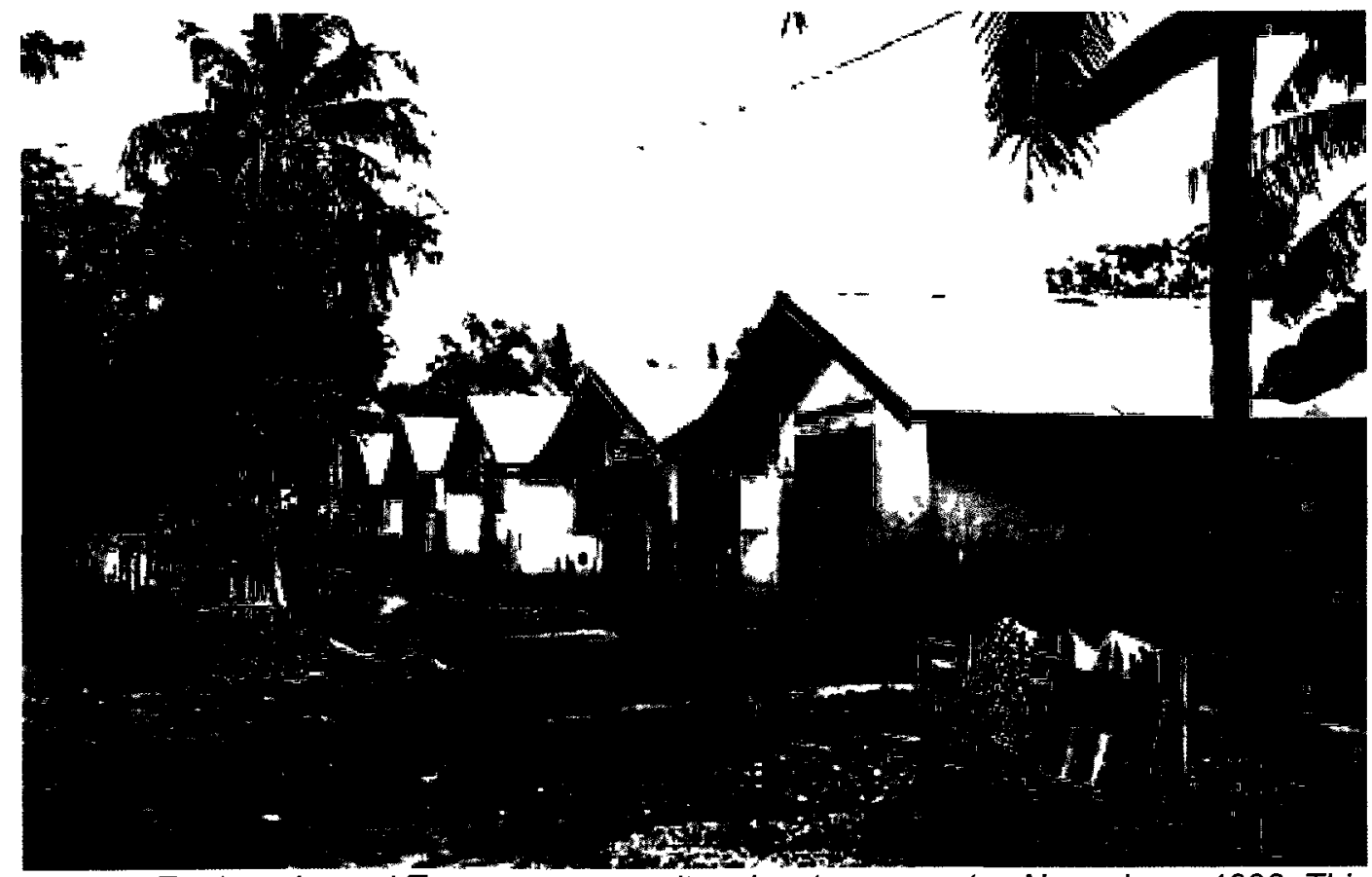

Fig. 1.5 - Earthquake and Tsunamı community relocatıon project in Nangahure, 1992 This image from 2001 depicts a vacant 'inland' village that has been abandoned by inhabitants having chosen to return back to their Wuring fishing village. 


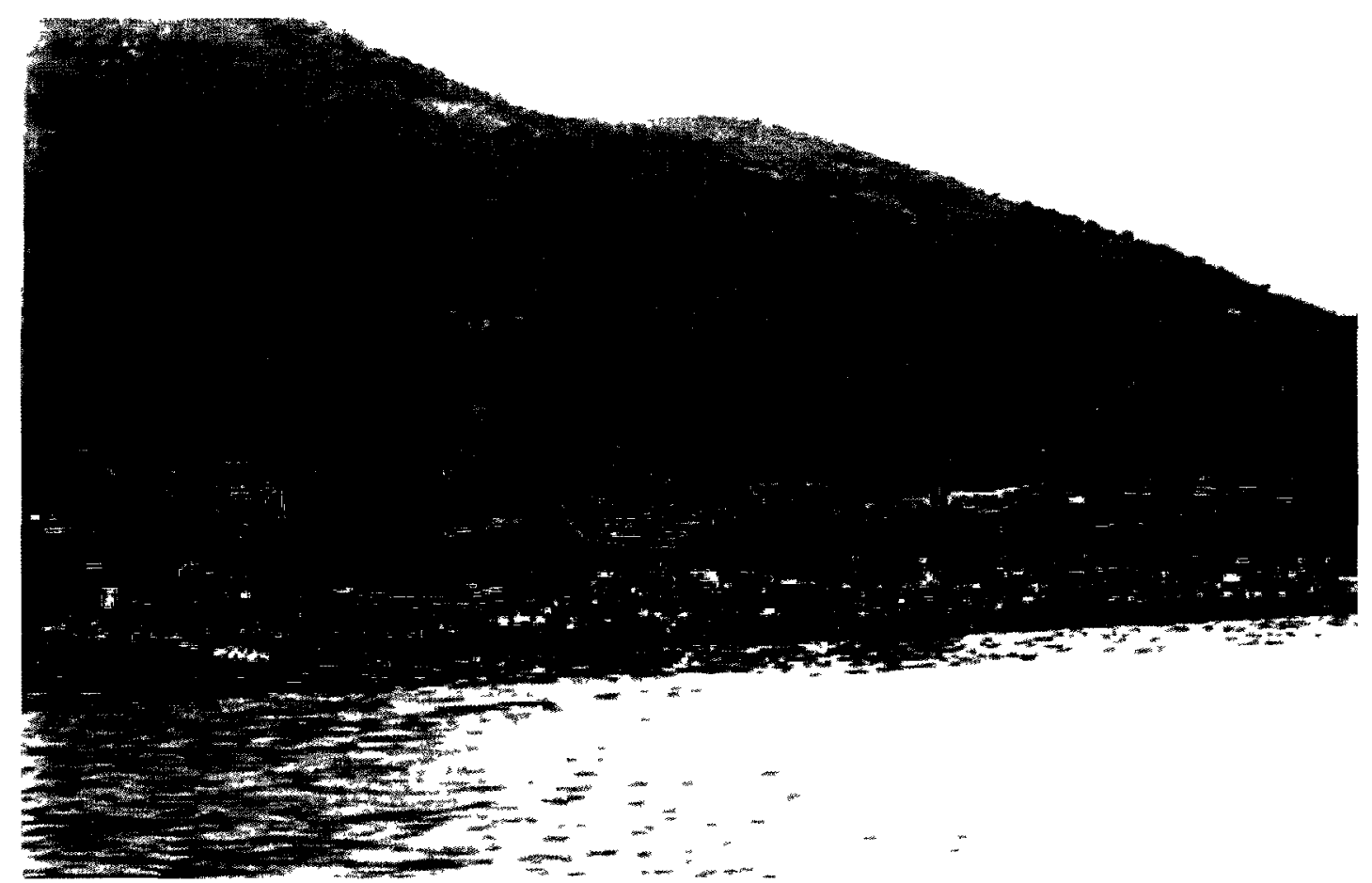

Fig. 1.6 - A 'new fishing village' built by the Babi Island fisherman on the shores of Nangahale, March 2001.

There were many critical community and cultural relationships that the 1992 Flores Island post-disaster rebuilding program neglected to address. In turn, their negligence resulted in an unsuccessful community resettlement project where community members decided it was better to pack up and move back to their original community, despite its condition.

This project clearly goes against all of Coburn's 'crucial factors' to successful community resettlement beginning with the first two factors: the physical environment of the new settlement and the relationship to the old village.

It is apparent in review of this case study that the geographical site was in direct opposition to the life that the Wuring people knew and lived before 
the natural disaster occurred. They were a fishing community who considered the sea a part of their life, so moving them to the inland community of Nangahure not only took them geographically away from their native community, it also robbed them of their culture and everything that would have been familiar.

Another key factor that was overlooked was the mixture of social and religious dynamics. There were two distinct religious groups: Catholics and Muslims. In their old village the reason they have lived in harmony for so many years is because they each had their own areas within the villages. With the new resettlement plan, the entire community was forced to live in the same barrack-like style of homes. In terms of 'their capability to develop their self,' they would be forced to learn agricultural ways of life if they were to survive inland, an enterprise that they knew very little about having come from a fishing community.

It is quite clear that adequate due diligence was not given, hence this resettlement project presents as an example of not having meshed cultural specific and localized information in response to a natural disaster rebuilding strategy. 


\section{Community Participation Explored}

Without the help of international aid and disaster response teams it would be nearly impossible for many developing countries to recover from natural disasters. And just as it is important to have outside organizations and international rebuilding groups be part of redesign and reconstruction efforts, it is also imperative that local groups, community members and local governments be involved to ensure that local perspectives are properly represented with similar end-goals in mind.

"The responsibility for positive social and environmental accommodation should not reside at the feet of professionals exclusively, however. It needs to be a collective enterprise in order to be embraced in an enduring and socially relevant way. Through partnerships among community groups, nongovernmental organizations, local governments, enlightened developers, academic programs, and architectural firms, a genuine engagement with the locale is more likely to produce a design response that is rooted in an understanding of the needs, mind, and spirit of another society". ${ }^{7}$

Collaboration begins to reveal itself as a complex dialogue of redesign strategy by and for the people, rooted within a local culture.

The success of a long-term rebuilding strategy within a developing context depends on the innovative thinking and organization of many different groups of local, national and international people. However, the most vital and often

\footnotetext{
${ }^{7}$ Kingston Heath. Vernacular Architecture and Regional Design: Cultural Process and Environmental Response (London: Architectural Press, 2009).
} 
underutilized participants are the beneficiaries; the local people who have lived before, through, and will now live after a natural disaster.

"The participation of users in up-front decision-making (within the project design and planning phases, including the capacity to make meaningful choices among a series of options offered to them) leads to positive results in terms of building process and outcomes. However, despite often-good intentions, this level of participation is rarely obtained and the capabilities of the users are often significantly wasted". 8

Involving the beneficiaries or 'users' who reside in the community where a disaster occurs only seems to make sense. These people would be able to translate how their culture once existed and validate truths and discredit falsehoods, but perhaps most importantly, they would be able to participate in the conception of their new way of life consistent with life from before a disaster. Also, by involving beneficiaries as active participants in all stages of rebuilding will help increase their capacities while reducing their vulnerabilities, thus bettering the chances of a long-term community solution taking hold. "Vulnerability can best be reduced by increasing people's capacity to deal with social, cultural, economic and physical factors". ${ }^{9}$

Capacities and vulnerabilities analysis play an important role in helping determine what groups of beneficiaries are most likely to succeed in any given post-disaster rebuilding strategy. If a disaster occurs in an area where the majority of the population works in construction, for example, it is likely correct to

\footnotetext{
${ }^{8} \mathrm{C} . \mathrm{H}$. Davidson et al. Truths and Myths About Community Participation in Post-Disaster Housing Projects (Habitat International 31.1, 2007) p.100-115.

${ }^{9}$ Nick Wates. The Community Planning Handbook: How People Can Shape Their Cities, Towns and Villages in Any Part of the World (London: Earthscan Publications Ltd., 2000) p.136.
} 
assume that labour capacities for rebuilding should already be quite strong and taken into account when formulating a rebuilding strategy. Perhaps the same group of construction workers are not as strong in the financial realization of their projects. This, therefore, would be considered a vulnerability and require they have more assistance in deciphering items like government loans and calculating interest rates. However, this example only refers to the physical/material realm of vulnerabilities and capacities analysis. There are three general categories within this analytical framework to consider: physical/material, social/organizational and motivational/attitudinal (Fig 2.1).

There is no hierarchy of importance to one category over the other. This is because they all speak to unique areas of identifying and gathering information to help formulate a long-term building strategy based upon local strengths and weaknesses. The physical/material realm refers to actual items or resources that have made it through the disaster, as well as localized skill sets that can help give direction to disaster response strategy. The social/organizational refers to political, social structure, coping systems and also to the need to identify any divisions that make a group of people more vulnerable; such as gender, race, language, class, etc. The third category of motivational/attitudinal refers to the individual "fighting spirit" ${ }^{10}$ or inner desire to want to rebuild, which is a key ingredient in establishing any successful endeavour involving a group of people.

\footnotetext{
${ }^{10}$ Mary B. Anderson \& Peter J. Woodrow. Rising from the Ashes: Development Strategies in Times of Disaster (London: ITDG Publishing, 1998) p.13.
} 
"To avoid increasing vulnerabilities, it is necessary to identify capacities in order to know what strengths exist within a society-even among disaster victims-on which future development can be built. Acknowledging the capacities of the affected population is essential for designing and implementing disaster responses that have developmental impacts". ${ }^{11}$

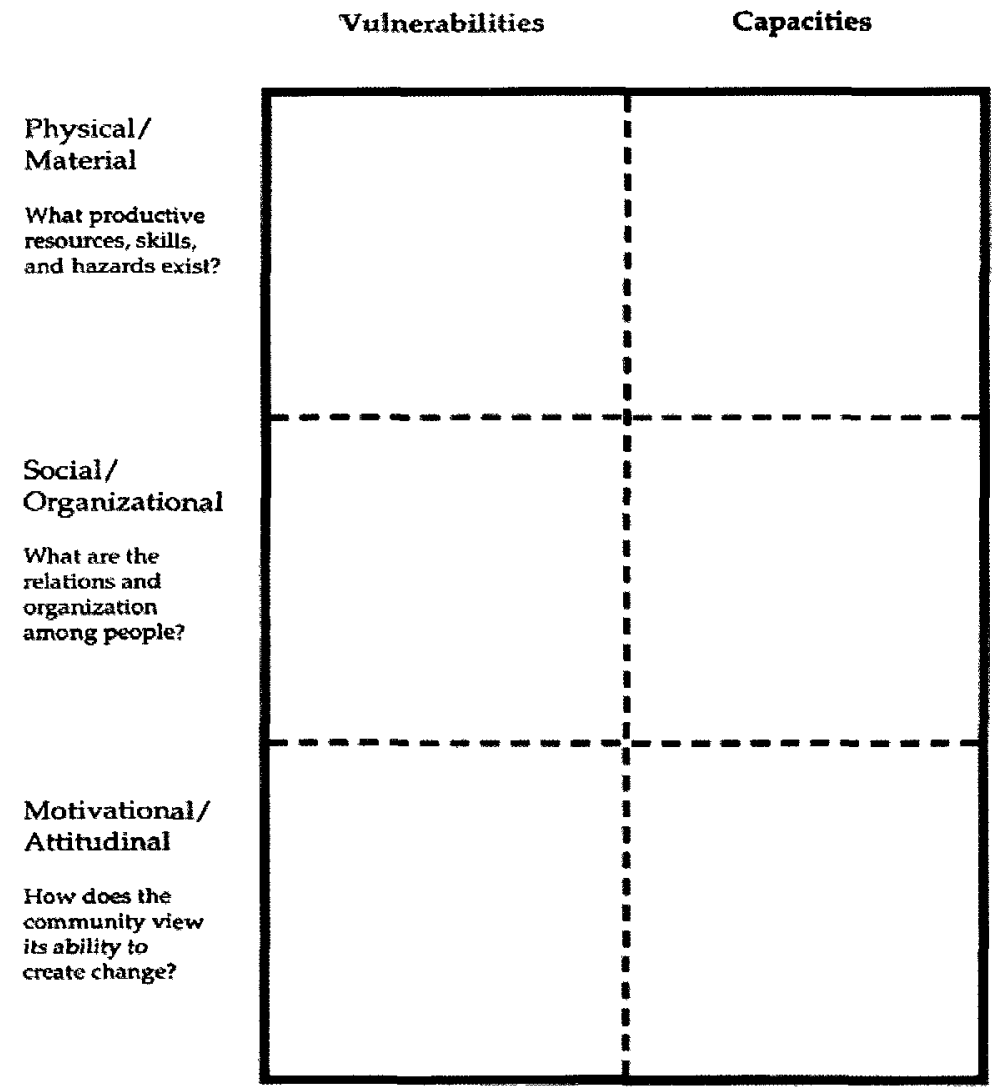

Fig. 2.1 - Vulnerabilities capacities and analysis matrix.

Regardless of where they exist in the analytical framework, both capacities and vulnerabilities should be identified and utilized towards an innovative long-term rebuilding strategy, making the beneficiaries' strengths even stronger and their weaknesses into an asset for improved post-disaster community life.

\footnotetext{
${ }^{11}$ Anderson \& Woodrow. Rising from the Ashes. p.11.
} 
When broken down, there are many rebuilding activity categories that beneficiaries can be involved in, all of which can help increase their existing strengths and weaknesses. ${ }^{12}$ Some of these post-disaster rebuilding activity categories include: program initiation, financing (loans, grants, etc.), project design, construction phase, post-project alterations and maintenance. All of these categories require delicate coordination and proper translation from one stage to the next and can benefit from utilizing local people in the accumulation of information and conception strategies, no matter the barrier of communication.

Language, religion, politics, gender, race-these are just a few of the many barriers humanitarian organizations face when dealing with foreign cultures, yet this does not often stop them from helping people in need; they adapt, get translators, research their ways of life, etc. The same concept can be applied to someone untrained in construction or design. Just because someone has had no formal training in these areas of expertise does not mean they will be unable to contribute.

A post-earthquake housing reconstruction project from Colombia in 1999 serves to illustrate how barriers of communication and education can be overcome. This rebuilding initiative required beneficiaries to take responsibility for all parts of the design and construction of their own homes and also to be in charge of approaching the local governing organization with their plans when complete. Engineers were used as the mediators to help the local people better realize their projects.

\footnotetext{
${ }^{12}$ Davidson et al, Truths and Myths. p. 100.
} 
"Most of the residents drafted a basic plan of what they wanted (by pencil and in a school-type notebook) while professional engineers provided advice for the design of the structural components for these customized solutions". ${ }^{13}$

\begin{tabular}{|c|c|c|c|c|c|}
\hline & case study & lombia & & & \\
\hline Activity/ actors & Benefficiaries & NGO & Govemment & $\begin{array}{c}\text { Hired } \\
\text { coniractions }\end{array}$ & Private firm \\
\hline $\begin{array}{l}\text { Program initiation (leading } \\
\text { in procuring the master } \\
\text { program of reconstruction) }\end{array}$ & & & & & \\
\hline $\begin{array}{l}\text { Project Initiation (leading ro } \\
\text { in startıng the project) }\end{array}$ & & & & & \\
\hline Project financing & & & & & \\
\hline Design & & & & & \\
\hline Construction & & & & & \\
\hline $\begin{array}{l}\text { Post-project modifications- } \\
\text { additions }\end{array}$ & N/A & & & & \\
\hline
\end{tabular}

Fig. 2.2 - Activity Categories/Actors chart from Colombia post-earthquake housing rebuilding initiative organized by the Coffee Growers Organization; Jan 25, 1999. This chart demonstrates how the CGO Rebuilding Initiative utilized the beneficiaries to their maximum potential.

${ }^{13}$ Davidson et al, Truths and Myths. p.105. 


\section{Community Collaboration \& The Art of Collecting Information}

Determining a strategy to collect consensus-based local knowledge is a key component to any long-term rebuilding strategy. There are many real-world cases of proven methodologies for collecting knowledge from large groups of people, including but not limited to; implementing public forums, planning workshops, design competitions, site modelling exercises, interactive displays and many more. However, careful consideration must be given to the selected mode of collaboration to ensure the information collection technique(s) are gauged specific to the community it is for. Post-disaster rebuilding solutions should be as unique as the culture they are strategized for. It therefore makes sense that context-specific community collaboration strategies should be a necessity. To demonstrate how success can be achieved via community collaborative planning routed in site-specific information and existing infrastructures, consider the following case study referencing a Colombian coffee growing post-earthquake community.

“On January 25, 1999 a magnitude 6.2 earthquake devastated a coffee growing region called 'Eje cafetero' in Colombia. In response to a preliminary master plan initiated by the federal government, the Coffee Growers Association proposed a rebuilding strategy utilizing their organization as a framework to mediate community reconstruction. "Having long experience working in the area and with local residents, the Coffee Growers Organization's 
started a few days after the disaster with the collection of data about the needs and priorities of the coffee growers". ${ }^{14}$

This is an example of how a specific post-disaster community already had a unique network of local, regional and national committees in place and were utilized in liaison with the reconstruction of the disaster areas. Community collaboration was organized around visiting a CGO centre that acted as an open public forum for advice and discussion to help develop specific strategies for rebuilding. For this specific post-disaster scenario 'the public forum' or 'open house' collaboration strategy was practical and successful because of its already trusted and established network of coffee organizations. Being able to identify and devise appropriate collaboration strategies is a crucial step in the rebuilding process, one that the CGO successfully implemented.

To better grasp the entire process of collaborative design, it can be divided into steps outlining specific areas of concentration that must be satisfied before moving to the subsequent step. Internationally recognized community planning reference Community Planning Handbook by Nick Wates will be used as the basic framework for understanding the process of community collaboration through a step-by-step approach. This will help to organize and better understand the many important decisions involved in selecting and implementing the correct collaborative approach. It will also help point to who should be involved in each step of a specific post-disaster or community resettlement planning strategy (Fig. 3.1).

\footnotetext{
${ }^{14}$ Davidson et al, Truths and Myths. p.103.
} 
According to Wates, defining the goal or purpose for gathering information is the first step. This means understanding the specifics of the scenario in hand: Was there a catastrophic event resulting in a need for community planning? If so the specifics of the disaster must be understood. Or perhaps the project is merely an attempt to obtain better living conditions for a group of people, in which case the focus would be more on the social dynamic and preventative design planning. In any case, this step deals with identifying the circumstances of the specific context and understanding the general goals wished to be achieved through collaborative design. The next category is about understanding the basic principles and design philosophies behind community planning.

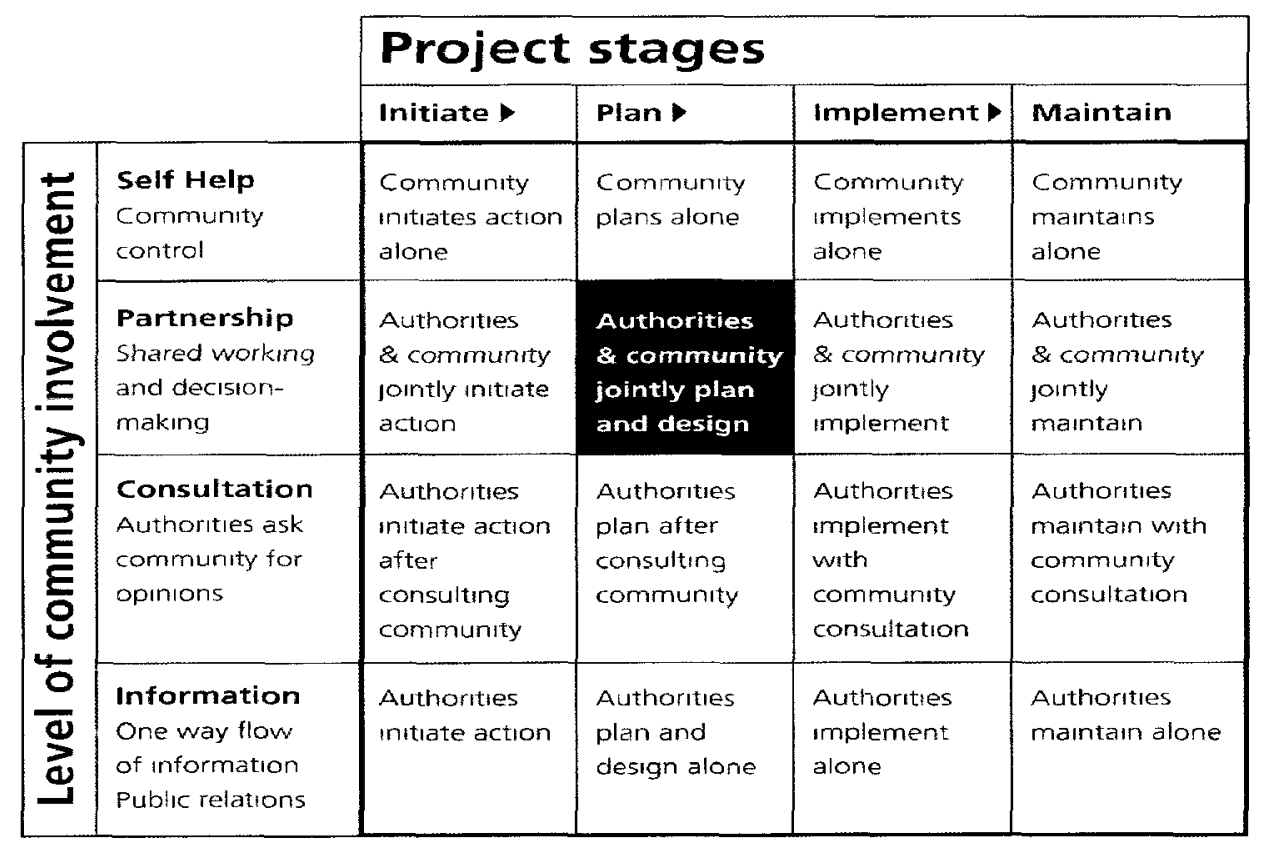

Fig. 3.1 - Nick Wates' Participation Matrix."A Simple illustration of how different levels of participation are appropriate at different stages of a project. Most community planning operates in the shaded areas. Any party may initiate action but the crucial ingredient is joint planning and design, shown in the dark square. Implementation and maintenance will either be carried out jointly or by the authorities after consulting the community." ${ }^{15}$

${ }^{15}$ Nick Wates. The Community Planning Handbook (2000) p.10. 
Wates' Community Planning Handbook identifies a number of general principles that can be applied to most planning situations. I have sorted through his list of design principles and chosen five general rules I feel best encompass the overall developing nation context of this research paper.

The first guiding principle is building local capacity. This has already been touched upon previously but its importance should be reinforced through its inclusion in this section. Using local help to plan and rebuild will increase local skill sets and leave communities with assets they can benefit from on a personal and communal level far beyond when rebuilding is complete. "The goal of participation is to encourage and enable people to build capacity and provide local stakeholders with the tools and techniques to effect change". ${ }^{16}$

The second design principle I have selected is to encourage collaboration via a mixture of methods. This stresses the importance of the necessity for collaboration in the first place and also promotes the diversity and innovation of collaboration techniques. Local people should be provided with accessible methods of participation that will not make them feel threatened should they want to participate. This also means encouraging a hybridized method of collaboration or several information collection techniques. Keeping an open mind and being flexible is essential. ${ }^{17}$

The third design principle is attributed to the importance of recording and documenting all events that take place while participating in collaborative design

\footnotetext{
${ }^{16}$ Sheri Blake. Participatory Design and Howard Roark: The Story of the Detroit Collaborative Design Center. Multimedia explorations in urban policy and planning beyond the flatlands (Dordrecht : Springer, 2010) p.226.

${ }^{17}$ Nick Wates. The Community Planning Handbook. (2000) p.14.
} 
exercises, as well as when living in or visiting the project community. Relevant information may not always seem useful at the time but having an accurate record of the who, what, where, why and how could become invaluable information in the later stages of a project.

The final two design principles again build on information covered previously and reference locality and cultural context, but here in this chapter are more specific to trust and respect. Respect for another community's culture means understanding the social, gender, economic, linguistic, political and casual mannerisms that exist within a group of people. In time a level of trust will generally be established as a result of maintaining respect, but until a mutual trust is in place the success of community collaboration could be in jeopardy. Once these basic principles of design are well ingrained the next step is to familiarize one's self with methods and case examples of past community collaborations.

It has already been identified that community collaboration strategies should be unique in order to responsibly select an approach for a specific community; due-diligence must be executed. This means understanding an overview of approaches that can been used and determining from these a direction for a specific community. Wates' Community Planning Handbook provides a list of close to sixty effective methods for helping people get involved in the physical planning and design process. These methods include everything from art workshops, interactive displays, prioritizing activities, planning weekends, and road shows to urban design studios, and the list goes on. 


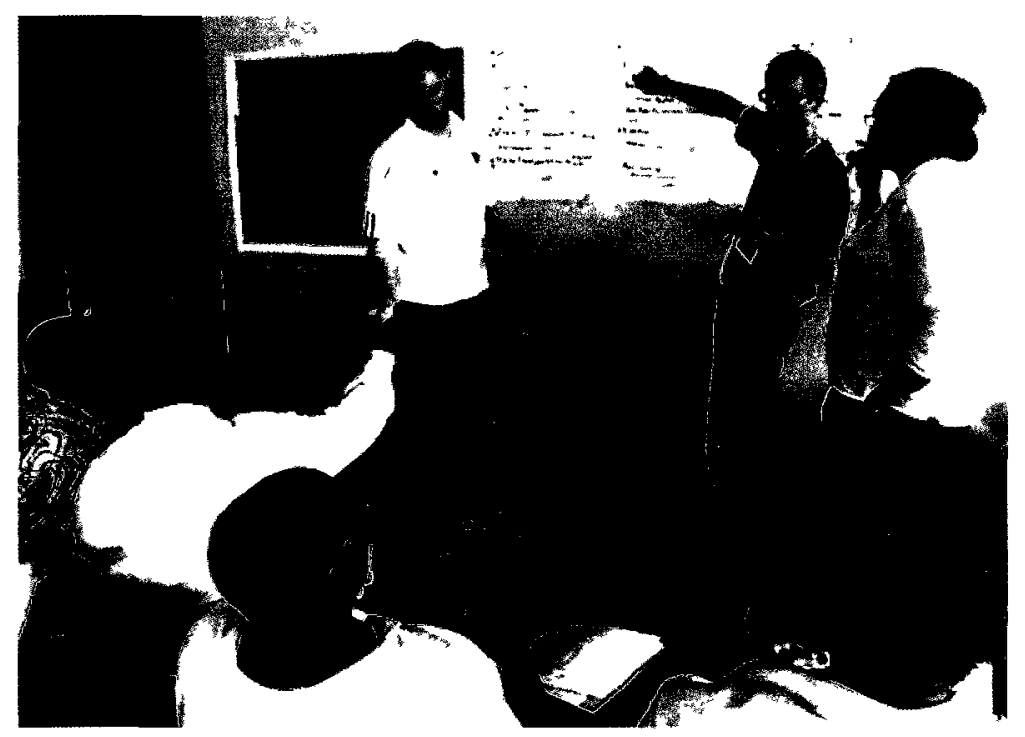

Fig. 3.2 - Participatory design prioritizing game. Each member of this workgroup was assigned four coloured sticker dots to place beside what they felt were the most important issues for rebuilding Léogâne. Rebuilding of Léogâne Design Workshop, Haiti: March 15-18, 2011.

One important piece of information that will dictate inclusion and exclusions when narrowing down and/or creating collaboration strategies is the importance of referring back to the context and social organization of the project community. Understanding project context will immediately help narrow down and flag methods of collaboration that may or may not work. An example of a third-world post-disaster context can be used to demonstrate this. The number of social and community organizational issues immediately increase exponentially in such scenario limiting options for the methods of collecting community information. Third-world concerns such as poverty, homelessness, literacy, disease and corruption are compounded when grouped with post-disaster immediate concerns such as first aid, shelter, community displacement, food and water. This threatens to compromise the accurate representation of an overall community through credibility issues when trying to democratically gather 
information. This means that community participation will need to be more selective and collaboration techniques such as an ideas competition, newspaper supplement, neighbourhood planning office or urban design studio would be immediately dismissed.

On the other hand, there are still many collaboration methods that can be considered. Such methods will inherently be more consultative and researchdriven but nonetheless continue to involve the participation of local community members. Some of these collaboration strategies include: prioritizing activities, risk assessments, design assistance teams, reconnaissance trips, field workshops and future search conferences. However, it should be reinforced that if the ideal scenario of a democratic representation from all geographic regions, social classes and religious segregations via collaborative design cannot be reached, then accurate consultant research and intervention based on local knowledge becomes that much more pertinent.

Even the smallest level of negligence in research or assumption has the potential to inhibit a community redesign strategy from taking hold:

"We need to be attentive to issues of social stratification in the communities we seek to aid. We cannot assume homogeneity even in the smallest of peasant villages. When houses and settlements are reconstructed in the aftermath of disaster, we need to recognize that materials and social space have profound meanings for people, meanings that divide and separate as well 
as unify communities. Reconstruction must be based on research which explores and discovers those meanings".18

In essence, all methods of community collaborative design are an attempt to uncover the nuances of specific society and their ways of life. It is the responsibility of the organization in charge of the project to verify and back up all assumptions and gathered information when moving forward. However, according to participatory design professional and scholar Sheri Blake, it is not enough to solely understand theory without exploring real-world examples of collaborative successes.

"Introducing the theories of participation to designers is not enough. It is critical to demonstrate good practice. To provide professionals and students of planning and design with an example of quality participatory design practice". ${ }^{19}$

In Sheri Blake's movie entitled Detroit Design Collaborative ... Amplifying the Diminished Voice, she documents the work of non-profit architecture firm Detroit Collaborative Design Centre (DCDC) in conjunction with the University of Detroit Mercy School of Architecture. The film follows the collaborative redesign process of a 28,000 square foot building for non-profit community based development organization Detroit Hispanic Development Corporation. The process used consists of a series of design workshops administered by DCDC in conjunction with the stakeholders from the project building. The workshops are meant to be

\footnotetext{
${ }^{18}$ A. Oliver-Smith. Post-Disaster Housing Reconstruction and Social Inequality: A Challenge to Policy and Practice, Disasters 14. (1990) p.17.

${ }^{19}$ Blake. Participatory Design. p.226.
} 
project-specific and in this example they were approximately three hours long, once a month, over a span of several months.

"In this case, workshops include the development of a project statement, a site walkthrough, a variety of design programming extending beyond conventional practice, an introduction to architectural language and its meaning, site tours of similar projects, literacy about how to read floor plans, spatial allocation modeling, digital mapping of the building and site, budget negotiations, design feedback sessions, and a presentation of the final work and package for capital fundraising purposes. A range of stakeholders, primarily building users, staff and board members, participate in the workshop series. Other representatives who may be involved in workshop processes include contractors, the architect of record, funders (bankers and/or foundations), technical assistance providers, and local residents who may be impacted by a development. ${ }^{20}$

Although this project is specific to a North American context, basic principles of participatory collaboration with architecture as the agent are demonstrated and can be adapted to other worldly examples.

After the above information is taken into account, the final steps in Nick Wates' Community Planning Handbook can be pursued. This will entail sketching out a scenario for your specific situation, producing an itemized budget, devising a plan, and lastly organizing a process planning session in order to begin collecting information. In the following chapters issues pertaining to context, planning sessions and collaboration details will be discussed specific to the project site of Léogâne, Haiti.

\footnotetext{
${ }^{20}$ Blake. Participatory Design. p.227.
} 


\section{Haiti, 2010: Historical Overview \& Rebuilding Context Explored}

The country of Haiti is historically unique in a number of ways. To begin with, it was the first independent nation in Latin America and the first black-led republic in the world to gain independence with their successful slave rebellion in 1804. It is also one of only two countries in the Americas with French as an official language (Canada being the other), which is a derivative of its roots as an established French colony in the $16^{\text {th }}$ century. Haiti is one of the poorest countries in the world according to the United Nations 2010 Human Development Index, which is a comparative analysis of life expectancy, literacy, education and standards of living for countries worldwide. Haiti is ranked near the bottom as a developing country coming in at 145 out of 169 participating countries and is estimated to fall in ranking even further this year, attributable to the aftermath from the 2010 earthquake. ${ }^{21}$

Haiti is geographically divided into ten large areas referred to as departments, which together make up a total of forty-two smaller regions called arrondissements. Haiti's land mass is 27,2750 square kilometres with a population of approximately fifteen million people concentrated in urban centres, coastal plains and valleys; the closest North American land size and population comparison would be the U.S. state of Maryland. Located in the Ouest Department approximately 30 kilometres west of the Haitian capital of Port-auPrince is the Léogâne arrondissement. This region has a population of

\footnotetext{
${ }^{21}$ United Nations. Development Report (2010) p.145.
} 
approximately 301,000 people spread throughout three towns; Léogâne, PetittGoave and Grand-Goave.

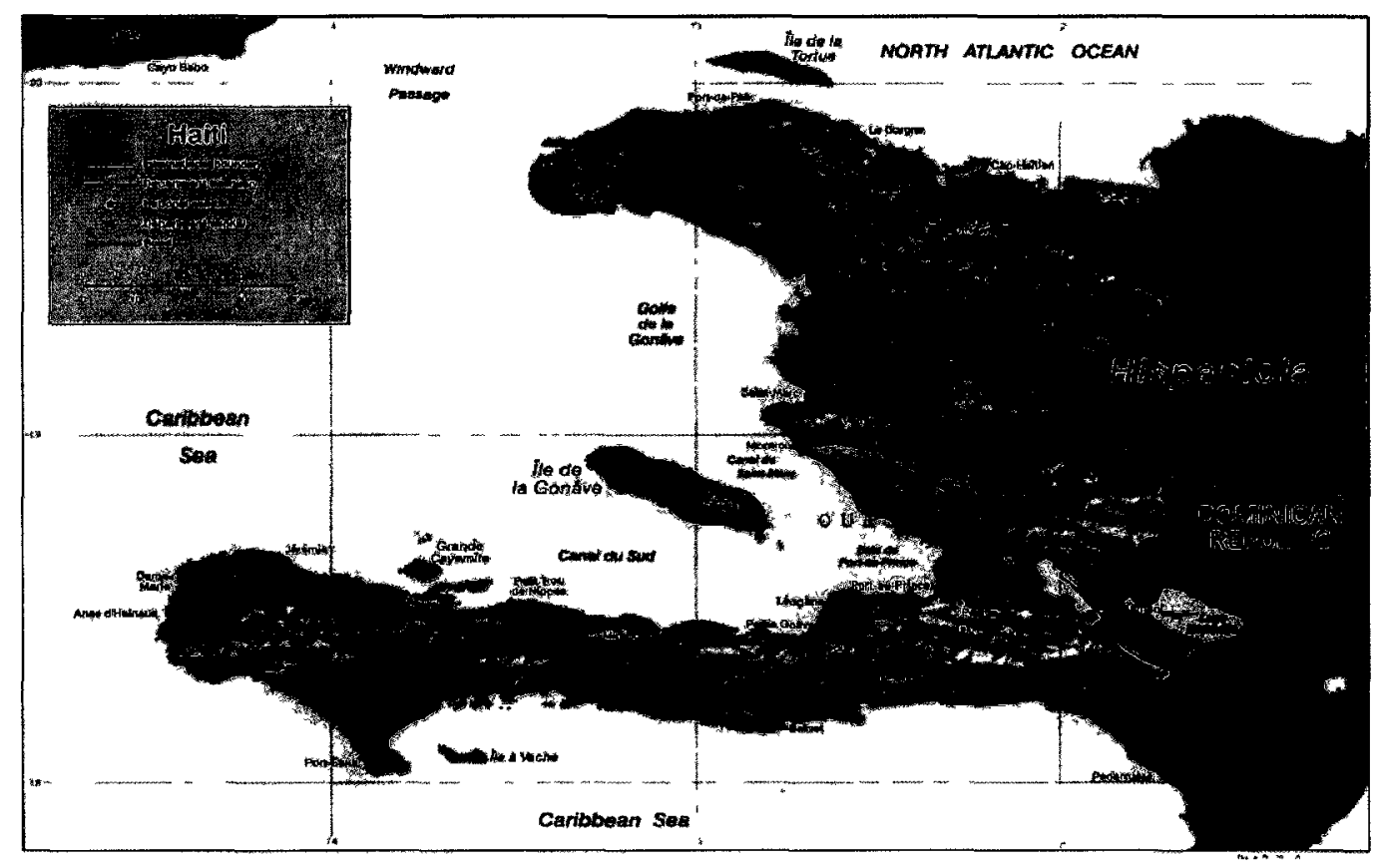

Fig. 4.1 - Shaded relief map of Haiti. This map shows Haiti's major geographical divisions which include (from largest in size to smallest): 10 departments, 41 arrondissements, and 133 communes.

When the January 12, 2010 earthquake shook Haiti it caused catastrophic damage throughout this developing country. The epicentre of the quake was located in the Léogâne arrondissement where damage was the worst, ranging from $80 \%$ to complete devastation. Historically speaking, this is not the first time this specific region was devastated by an earthquake. In 1770 the Léogâne arrondissement's soil was reportedly 'liquefied' and completely destroyed in a magnitude 7.5 earthquake. Since the 2010 earthquake a large number of international aid donors, local government officials and non-governmental organization (NGO) support groups have been mitigating relief efforts and 
rebuilding strategies throughout the disaster struck areas. The main earthquake, together with a recorded 52 smaller aftershocks, was felt nationwide along with one reported small localized tsunami. ${ }^{22}$

The specific site of my research will be located at the heart of the 2010 Haitian earthquake; in the small town of Léogâne. I decided early in my research that my focus would be rooted in both a post-earthquake and developing country context. Choosing to use the Léogâne arrondissement as the site for my project appears an appropriate choice as it makes sense that the best way to explore post-disaster rebuilding is to choose a site where the adverse effects are strongest.

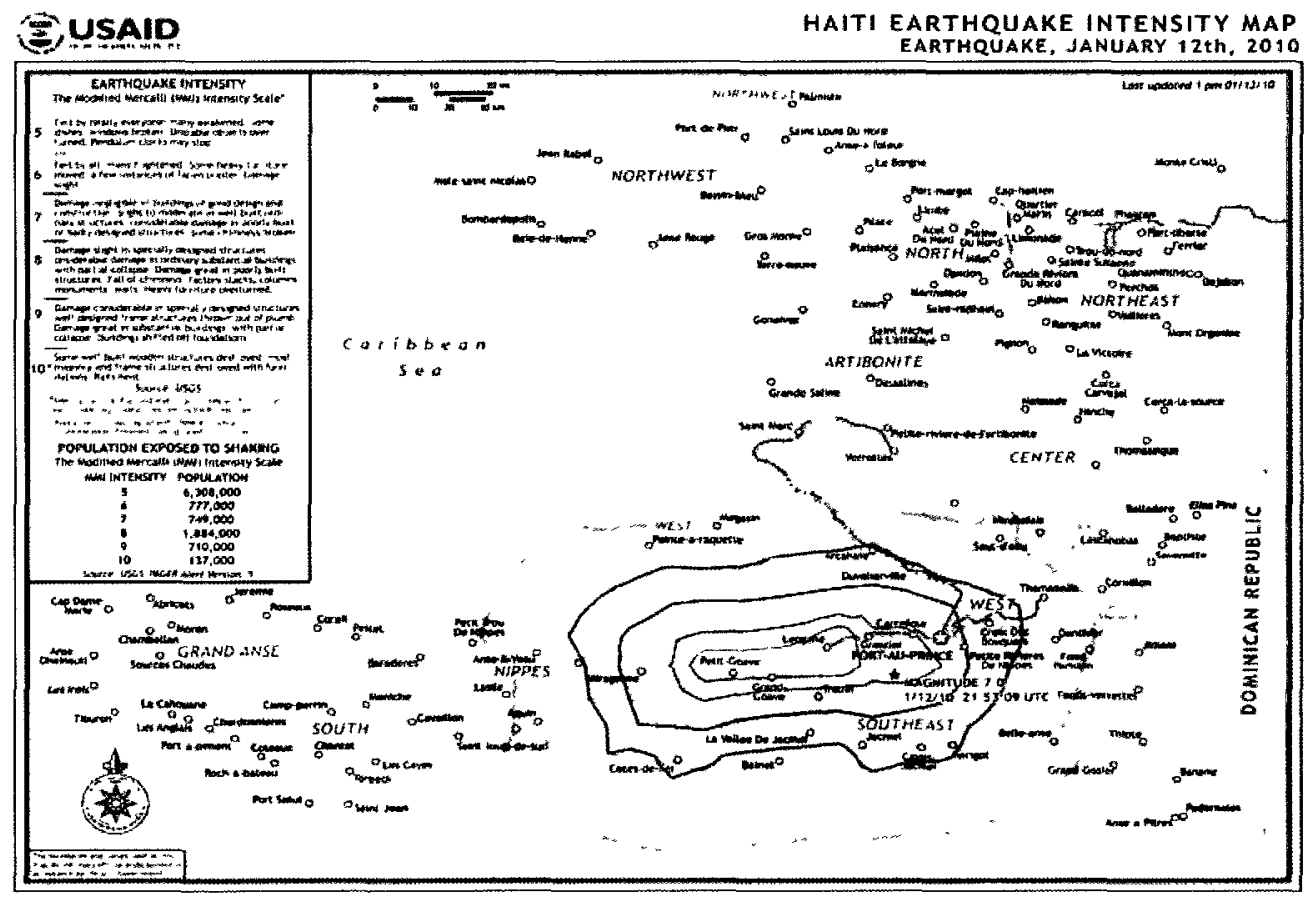

Fig. 4.2 - Earthquake intensity map from January 12, 2010 Haiti. The Léogâne arrondissement (part of the west department) is located within the center intensity ring of this map representing the epicentre of the earthquake.

\footnotetext{
${ }^{22}$ Haitian Government. Action Plan.
} 
In March 2010 the Government of the Republic of Haiti issued a document entitled Action Plan for National Recovery and Development of Haiti which addresses immediate to long-term developmental issues for the future of the country. The Haiti Action Plan 2010 is based on the ideas and opinions of not only local Haitian sectors, but is also informed by the international community and many outside consultants.

"The plan we are putting forward is based on a joint effort of reflection and consultation. In diplomatic circles, formal and constructive talks have made us aware of the expectations of our international partners and allowed us to explain to them our choices for the future". ${ }^{23}$

Another integral contributor to the Haiti Action Plan 2010 is taken from the Postdisaster Needs Assessment (PDNA), which is a standardized document for postdisaster scenarios developed by national and international officials in determining damage, losses and requirements.

The Haiti Action Plan is divided into several sections, beginning with introductory information that includes a PDNA summary, vision and approach for Haiti's rebuilding, and immediate actions required. The bulk of the report exists in a framework established by the Haitian government whereby they break down reconstruction into four main categories: territorial rebuilding, economic rebuilding, social rebuilding and institutional rebuilding. The report then concludes with a macroeconomic framework for 2010-2015, funding mechanism and management and reconstruction structures.

\footnotetext{
${ }^{23}$ Haitian Government. Action Plan. p.3.
} 
The portion of Haiti's Action Plan this research is most concerned with is its long-term vision for 2030_'to be an emerging independent nation'. The plan's restructuring strategy is marked by a number of fundamental principles:

"A fair, just, united and friendly society living in harmony with its environment and culture; a modern society characterized by the rule of law, freedom of association and expression and land management. A society with a modern, diversified, strong, dynamic, competitive, open and inclusive economy based on the land. A society in which peoples basic needs are met both quantitatively and qualitatively. A knowledge-based society with universal access to basic education, mastery of qualifications based on a relevant professional training system, and the capacity for scientific and technical innovation fed by a modern and efficient universal system, in order to create the new type of citizen the country needs for reconstruction. All of this, under the supervision of a responsible, unitary state guaranteeing the implementation of laws and the interests of the people with a strong commitment to de-concentration and decentralization". ${ }^{24}$

On more of a macro level, two encompassing themes have been selected that help put Haiti's fundamental rebuilding principles into perspective: vulnerability reduction and local, national and international connectivity are portrayed as essential in laying a solid foundation for the future of Haiti.

As discussed in chapter II, vulnerability and capacities analysis is an important analytical tool used for determining strengths and weaknesses of

\footnotetext{
${ }^{24}$ Haitian Government. Action Plan. p.8.
} 
any given community of people. The goal of this analytical tool after identification of vulnerabilities is to figure out how to expand capacities of a project community so as to leave them with a decreased amount of vulnerabilities after rebuilding has occurred. In the case of Haiti's Action Plan, the need to decrease vulnerabilities is priority; this will not only allow Haiti to grow economically and become more independent, but it will also increase its readiness thus minimizing potential damages from future natural disasters.

The second main theme put forth in Haiti's Action Plan is its need to connect itself on a community, national and international level. Its concern for connectivity is addressed through a desire for more roads, ports and airports, but also through a desire for better access to public services, social development, education and healthcares services. The desire for connection is not only demonstrated through a literal sense, but also via a representational sense through its understanding of the importance and benefits of joining a global community. "With this in mind, we must strengthen the links between all regions of the country and encourage the strengthening of the regional partnerships that will create opportunities for change all over the country, the Caribbean and beyond". ${ }^{25}$ This global connection will help Haiti stay in sync with important issues such as storm warnings and medical advances, providing Haiti the opportunity to represent their identity on an international platform.

\footnotetext{
${ }^{25}$ Haitian Government. Action Plan. p.3.
} 


\section{Project of Architecture: Hypothesis \& Pre-Haiti Development}

To properly situate the final project within the framework of this paper, a synopsis of essential rebuilding information from all previous chapters will be required. This will then be used to better position a preliminary design proposal for a project of architecture within the disaster-struck community of Léogâne.

Accordingly, this research begins by defining the term cultural resilience as 'the power or ability to return to the original form' and 'the ability to recover readily from illness, depression and adversity.' This terminology is a point of departure, but more importantly it is a driving theme that links the various sections of this research together. The chapters are arranged to lay foundation to suspected key contributors for long-term community redevelopment via architecture as the agent, beginning with the importance of local knowledge.

The importance of addressing 'regionalism' within a community is then discussed as the ability to utilize vernacular strategies as key components in rebuilding projects. These vernacular strategies are a community specific response and are not always as readily available as in the 2005 Pakistan Earthquake case study, where the ability to identify and use an age-old 'earthquake resistant' building technique was a viable option. It is also important to remember 'vernacular' does not necessarily imply a technical solution, but rather normative elements of a community's culture left up to interpretation and discovery via collaboration. Since Haiti is such a poor and developing country, one of the projected redesign challenges will be to identify vernacular 
consistencies within its infrastructure and existing building techniques.

This is where the community participation section of research becomes more relevant, which focuses primarily on the 'beneficiaries' and their often underutilized capacities in rebuilding initiatives. The importance of community input is paramount in identifying local histories and capacities that could potentially be used to rebuild.

Nick Wates' community collaboration research is explored in the section highlighting his general principles, which include building local capacity, using many different collaboration strategies, recording all events, and engendering trust and respect. Final steps are also talked about that reference project specifics, such as sketching out a scenario for your own situation, devising a plan, and lastly, organizing a process-planning session to begin collecting information. Most of the information gathered for chapter VII, Project of Architecture: post-Haiti development, has taken place while visiting Haiti, but prior to that point the Haiti Action Plan was used to inform and create an initial theoretical position towards a final project of architecture.

The preliminary title of my project was The Haiti Information Exchange. This project of architecture, a mixed-use, public facility that was meant to act as a threshold between the people and two key issues for rebuilding that have been identified in the Haiti Action Plan in chapter IV, Haiti, 2010: Historical Overview \& Rebuilding Context Explored: connectivity and vulnerability.

Connectivity, the first issue, inherent in this modern day community centre with large covered gathering spaces for people to congregate and connect on a 
community level. It was intended for any occasion, as a place to celebrate the arts and culture, a centralized location to make important public announcements, performance stages for festivals, market space for vendors or even agricultural kiosks for farmers' markets. In terms of national connectivity, this community facility was thought to have public broadcast screens with live camera feeds to different parts of the country that would be seen at all times of the day. These broadcast areas were also to be used for gatherings, celebrations, and breaking news reports, but most importantly, for providing a transparency and feeling of connection for all community members to different parts of their country. Since Haiti is such a poor nation the majority of people would likely never have a chance to visit different parts of their relatively small island. This medium would have allowed them the opportunity to see and hear beyond the geographic limits of their own community. Finally, an international connection component via Internet and news station kiosks. In this proposal, individual and interactive kiosks would have been made available to give people the freedom and independence to reach out and connect with the rest of the world.

The second guiding principle that fuelled the development of the preliminary project was the theme of vulnerability reduction. As previously ascertained, a decrease in vulnerability translates to increased capacity, which means a more informed population with better equipped mechanisms and knowledge systems. These are essential components for rebuilding a nation, especially a developing nation faced with such devastation. It is imperative that skill sets are expanded upon, and in response to this need the community centre 
will have space dedicated to training facilities. The local skill-sets developed here would be based on localized techniques and strategized solutions that could also be practical and efficient for other areas of their community and country.

Adding another layer of vulnerability reduction to the proposed project is the potential of this community facility project to double as an evacuation station. Imagined as a raised platform-type of construction open to larger spaces below, this structure was purposed to house the equipment necessary for staff to issue evacuation and storm warnings. Community members would have had access to this building for refuge, buying them time and providing safety from future natural disasters.

On a conceptual level, this hypothesis driven preliminary study was an organization of large outdoor spaces and platforms that would be covered from above by some sort of canopy or roof structure. I envisioned a number of smaller kiosks used as partitions to separate the different programmatic elements of this project.

A number of geographic locations were identified as potential project sites, but due to lack of maps and inconsistency of community documentation, the final site selection was to be confirmed during the community site visit. It made sense that the site for this project was a central location within the community of Léogâne to allow for close proximity and accessibility for all people. Other important aspects included location on a main road to allow people visiting the city to easily locate the site or perhaps choose a site that needs to be crossed on the way in or out of town. Another important project site determinant that came 
up was current and past land use. The site should needed to be a socially, religiously, politically suitable site selection for the intended end use of a 'market type' program. The 'Rebuilding Léogâne' design collaboration assisted in identifying a better understanding of the overall dynamic of the town.

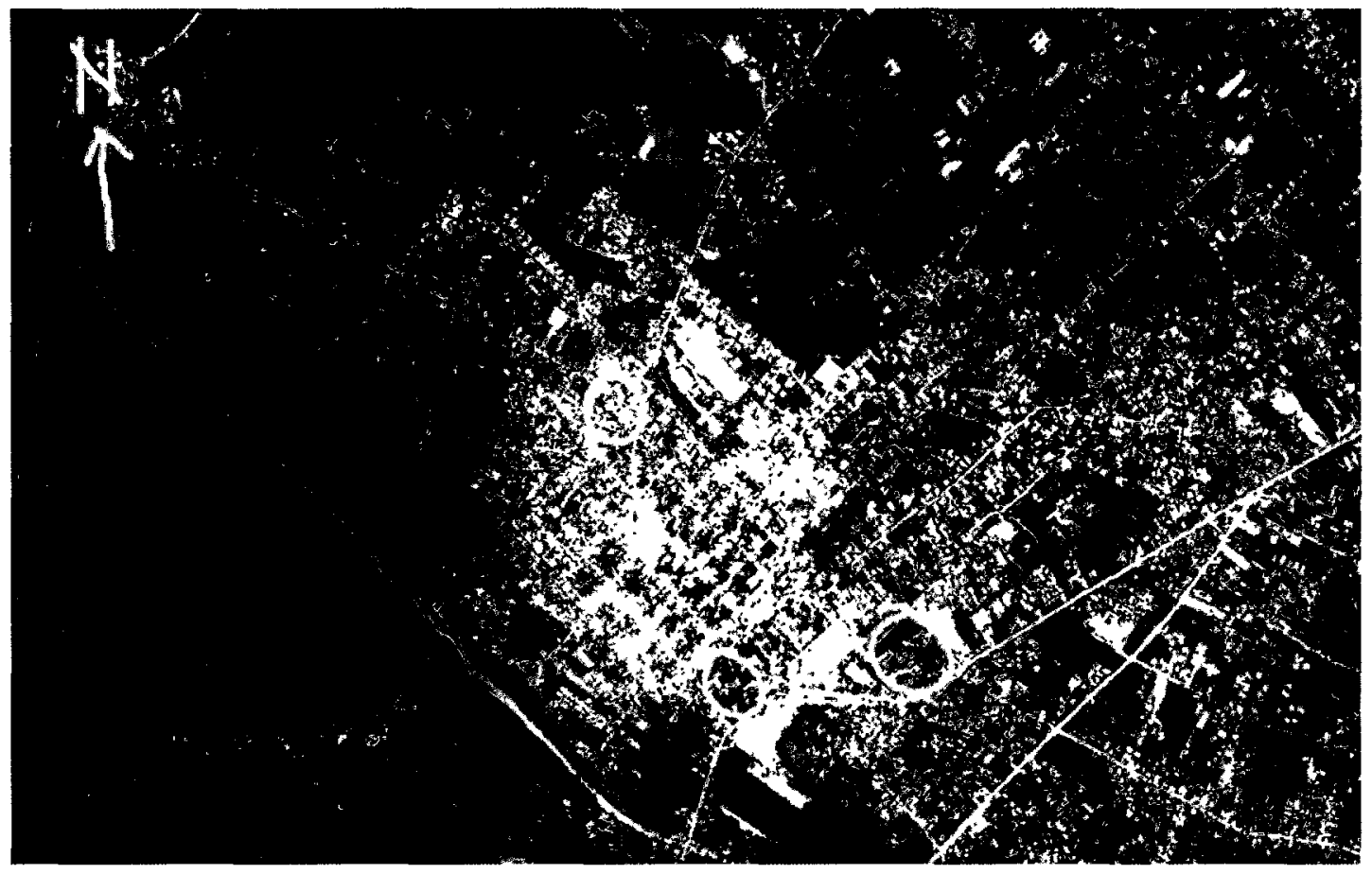

Fig. 5.1 - Town of Léogâne, Haiti: aerial photograph. Preliminary site selection map.

The driving themes of vulnerability and connectivity dictated early in the design phase what this project was to entail and roughly how it would be organized. This preliminary project study was not able to move past organizational strategies until the site visit occurred in March 2011 when essential cultural and site specific information was gathered. 


\section{Haiti: Léogâne Design Charette \& Local Culture Explored}

In March 2011 I travelled to Haiti with non-profit charity 'Schools for Children of the World' in order to participate in a master planning design charette for the town of Léogâne. The event consisted of a multi-day series of collaborative design workshops covering topics such as housing, roads, water and sewers, utilities, education, health services, police and fire, commerce, parks and green space, building codes, and other public services such as garbage collection, cemeteries, etc. The design workshop proved to be an invaluable validation tool for this research as it has substantiated hypothetical information and translated it into important local knowledge and collaboration actualities. The design workshop combined with site visits has allowed me to better align my research and final project to address the specific needs within Léogâne, especially in reference to culture and human capital.

The Rebuilding of Léogâne' Design Workshop was a four-day interactive collaboration that took place March 15-18, 2011 at the University of Notre Dame research facility in Léogâne, Haiti. This design and planning conference brought together local community stakeholders (school principals, nurses, engineers, business people, artists, musicians, etc.), local rebuilding organizations (C.U.R.L), major international aid organizations, and NGO's (Red Cross, U.N., etc.).The University of Notre Dame acted along with Schools for Children of the World (SCW) as the organizational bodies for the workshop. The mandate for this workshop was to organize ideas and 
efforts from the local and international community in order to help devise a holistic rebuilding plan for this town that was nearly destroyed in the 2010 earthquake.

Local community participation was prioritized as an essential part to this entire process, and there was certainly no lack of representation at this planning event, attracting three-quarters of the approximately 150 people in attendance from the surrounding area. Having such a strong showing of community representation also added to the credibility of information collected, making it more a democratic consensus rather than a calculated assumption based on a few opinions.

Although the mix of attendees was from disparate backgrounds and represented divergent interests, the workshop itself was formally structured and followed a pre-set agenda. To begin there was a general introduction by the organizing bodies, SCW and Notre Dame, after which participants were organized into nine randomly selected sub-groups. This was a chance for townspeople to express concerns and discuss not just their areas of expertise, but to also comment on the community as a whole and identify issues they felt needed attention and to present prioritized lists based on their thoughts. The intention of breaking into groups so quickly was to ensure that local ideas were the driving force behind this workshop and to have this known to all workshop participants. The first day for non-local persons was about listening, understanding and allowing for local people to set the tone for the week.

"Our involvement in the rebuilding of Léogâne is predicated on the belief that 
its future should lie in its people's hands and should not be dictated by any single individual, foreign or domestic. While the long-term objective will be to deliver a better quality of life to its people, which improves upon the conditions prior to the quake, it should not presuppose the form that solution or vision will take. Instead we approach this problem as listeners first, offering ourselves as a vehicle that brings our technical knowledge, experience, resources, and commitment to Léogâne to help realize this future through an integrated approach that promotes transparency, participation, and respect for all stakeholders". ${ }^{26}$

At the end of the first day short group presentations were put forth, which prioritized a consensus of primary directives for a rebuilding strategy within Léogâne. Although many priorities were brought to the table, it quickly became clear that the long-term needs were held in common and continually repeated throughout these presentations: the demand for a better healthcare system and facilities; the need for accessible public education for everyone; and improved water and sanitation systems, to name just a few. The following two days of the workshop were dedicated to again asking focus groups to prioritize both long- and short-term goals for the community within their specific areas of expertise.

The second and third days were also a chance for workshop participants to explore more deeply into their areas of expertise by choosing one of eight workshop focus groups to take part in. The eight groups were broken down thusly: economic development, education, health, housing,

\footnotetext{
${ }^{26}$ Notre Dame Univ. Rebuilding Léogâne Planning Workshop.www.nd.edu/ ce4haiti/. (Jan. 2011).
} 
infrastructure, planning and zoning, public service, and culture. Again, each group was comprised of local community stakeholders and NGO participants who worked together to first understand the issues and then to generate feasible ideas for both the long and short term.

The fourth and final day of the workshop provided each group the opportunity to present their priorities to the larger group and explain how their particular expertise fit into their vision of an overall master plan for Léogâne. The theme of interconnectivity began to reveal itself as the presentations progressed, demonstrating that all areas of community rebuilding should be inclusive in order to generate any sort of practical and cohesive planning strategy.

The primary formal deliverable from this workshop will eventually be a 'Léogâne Community Action Plan' which is currently being assembled into a final report that is an accumulation of written documents from different expertise work groups.

"Formulation of an Action Plan that includes the short- and long-term vision for Léogâne, recommendations for essential elements of Léogâne's Master Plan, and requirements for successful implementation, based on the collective input and knowledge generated by this Planning Workshop". ${ }^{27}$

This document is intended to align an overall vision and to be used by consultants to develop designs rooted in the best interest of the community.

Another essential component of my research validated through this

\footnotetext{
${ }^{27}$ Notre Dame Univ. Rebuilding Workshop Website.
} 
workshop was the importance of working with local persons as they in fact are the ones with the ability to identify critical community concerns.

In the 'Healthcare' group was ministry of Haiti health representation, local nurses, and the dean from the Episcopal Nursing School, all of whom could identify specific local information such as how many hospital beds were really available in the Léogâne area (approximately 126 for 230,000 people) ${ }^{28}$, and the dire need for maternity hospitals (550 pregnancies per month in the Léogâne community alone, of which only a small percentage are actually detected and properly monitored) ${ }^{29}$

Within the 'Education' group were several local principals and teachers who could speak directly to the problems the children and school system is facing. First and foremost is that there is no widely accessible public school system in Haiti. However local teachers spoke firsthand about the finer details directly affecting the children: poor learning conditions and temporary facilities, lack of school supplies, lack of water for the children while they are at school, etc. ${ }^{30}$

In the 'Culture and Human Capital' group were two local fine artists: one clay sculpture artist (arts plastique) and two musicians. These artists and artisans were given a platform within the workshop to voice the historical, social and cultural significance of their work within their local and national communities. A local musician (Sammy Jean-Baptiste, lead singer

\footnotetext{
${ }^{28}$ Rebuilding Léogâne, Healthcare Group. Notre Dame Research Facility Haiti. (March 2011).

${ }^{29}$ Healthcare Group. Notre Dame Haiti. (March 2011).

${ }^{30}$ Rebuilding Léogâne, Education Group. Notre Dame Research Facility Haiti. (March 2011).
} 
of a local Rara band) shared with us the importance of a music genre and celebration called 'Rara'. This is a popular public festival within the Haitian culture and for the town of Léogâne which is the destination for the final celebration to close the Rara season; attracting approximately 36 local bands. ${ }^{31}$

"Rara is the yearly festival in Haiti that, even more then Carnival, belongs to the so-called peasant classes and the urban poor. Beginning the moment Carnival ends, on the eve of Lent, and building for six weeks until Easter Week, Rara processions walk for miles through local territory, attracting fans and singing new and old songs. Bands stop traffic for hours to play music and perform rituals for Afro-Haitian deities at crossroads, bridges and cemeteries". ${ }^{32}$

Although this celebration is rooted within the religious and spiritual realm, Sammy Jean-Baptiste informed us the festival is very much about culture and singing about historical, social and political events from the past and present in Haiti. Author Elizabeth McAlister addresses the cultural significance of the Rara music in her book entitled Rara!: vodou, power, and performance in Haiti and its diaspora.

\footnotetext{
${ }^{31}$ Rebuilding Léogâne, Culture Group. Notre Dame Research Facility Haiti. (March 2011).

${ }^{32}$ Elizabeth A McAlister. Rara!: vodou, power, and performance in Haiti and its diaspora. (University of California Press, 2002) p.3.
} 


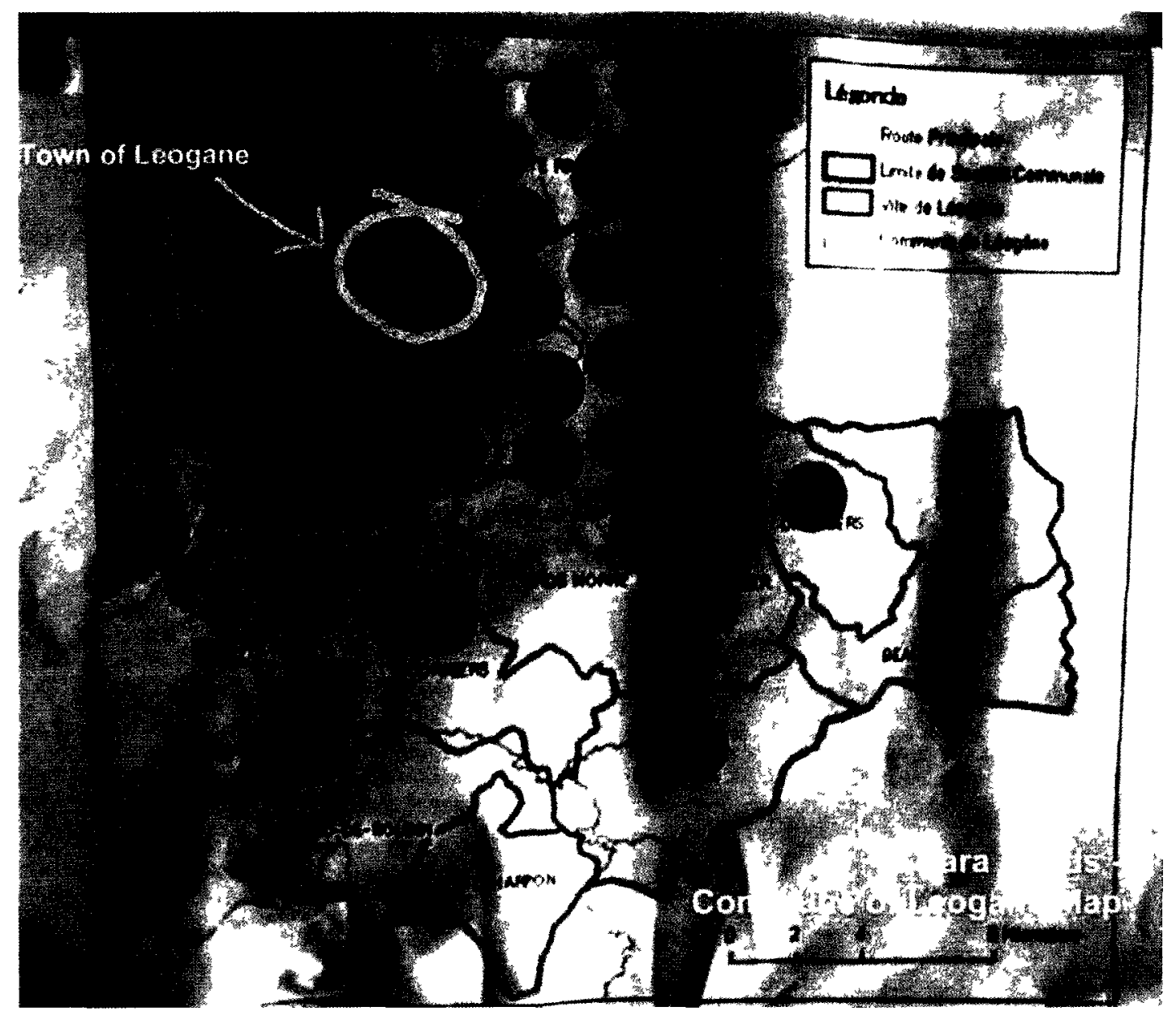

Fig. 6.1 - Map of Rara Bands within the Léogâne comune. This map was created by the Culture Group local participants as part of the 'Rebuilding Léogâne Design Charette, March 15-18, 2011.

"My analysis of Rara derives from the conviction that rituals are primary documents that can be 'read' just as well as books in a library and that can reveal as much about culture as any written text. I treat older Rara songs as historical and social texts that people have used for generations to reveal truths about the moment at hand". ${ }^{33}$

\footnotetext{
${ }^{33}$ Elizabeth McAlister. Rara! (2002) p. 14.
} 
Musician Sammy Jean-Baptiste also explained there are many forms of participation in Rara and just because you are not in the band or not religious does not mean you cannot participate. Many people that do not feel like being part of the musical parade will line the streets to watch as they pass. As expressed above, it is very much about creating a 'social text' of an era which acts as a medium for the people to address current and past issues within the Haitian culture.

The fine artists participating in the Rebuilding Léogâne Design Workshop brought in examples of their artwork to demonstrate how they translate culture through their various art forms. Similar to how Rara music captures the essence of Haitian culture through sound, the fine art pieces as shown below convey similar social, historical and cultural topics in the physical form.

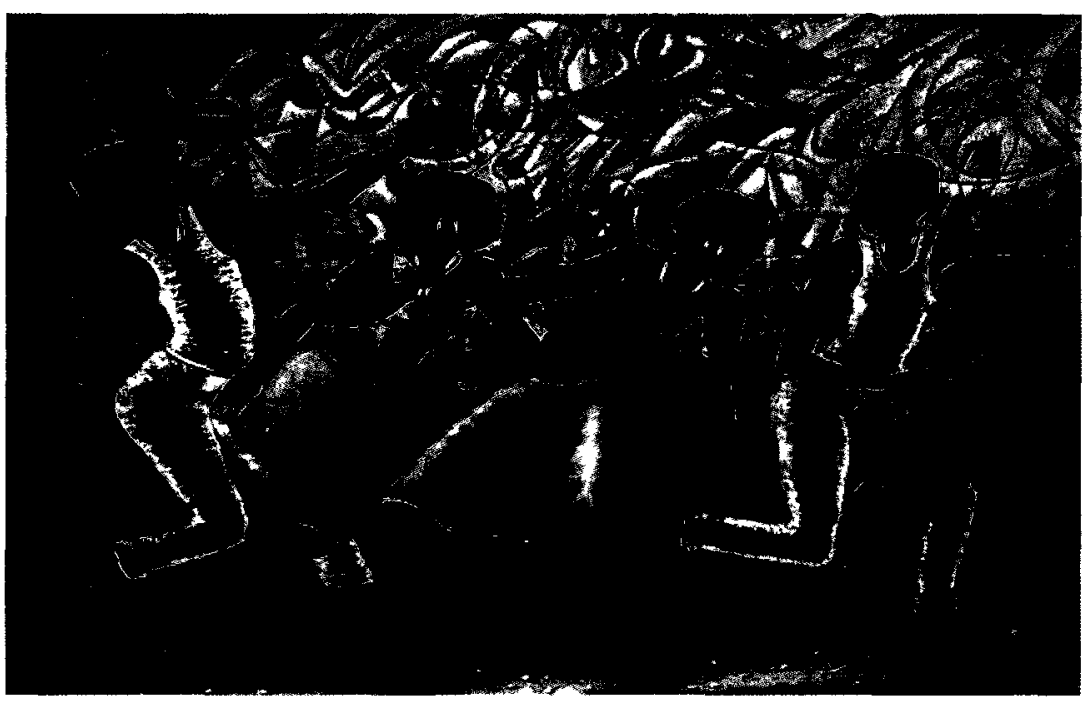

Fig. 6.2 - Pastel and Acrylic Painting by Haitian Artist Jean-Eddy Beauvoir. This painting depicts people dancing, drinking and playing instruments attempting to capture the atmosphere of a local Rara celebration in Léogâne, Haiti. 


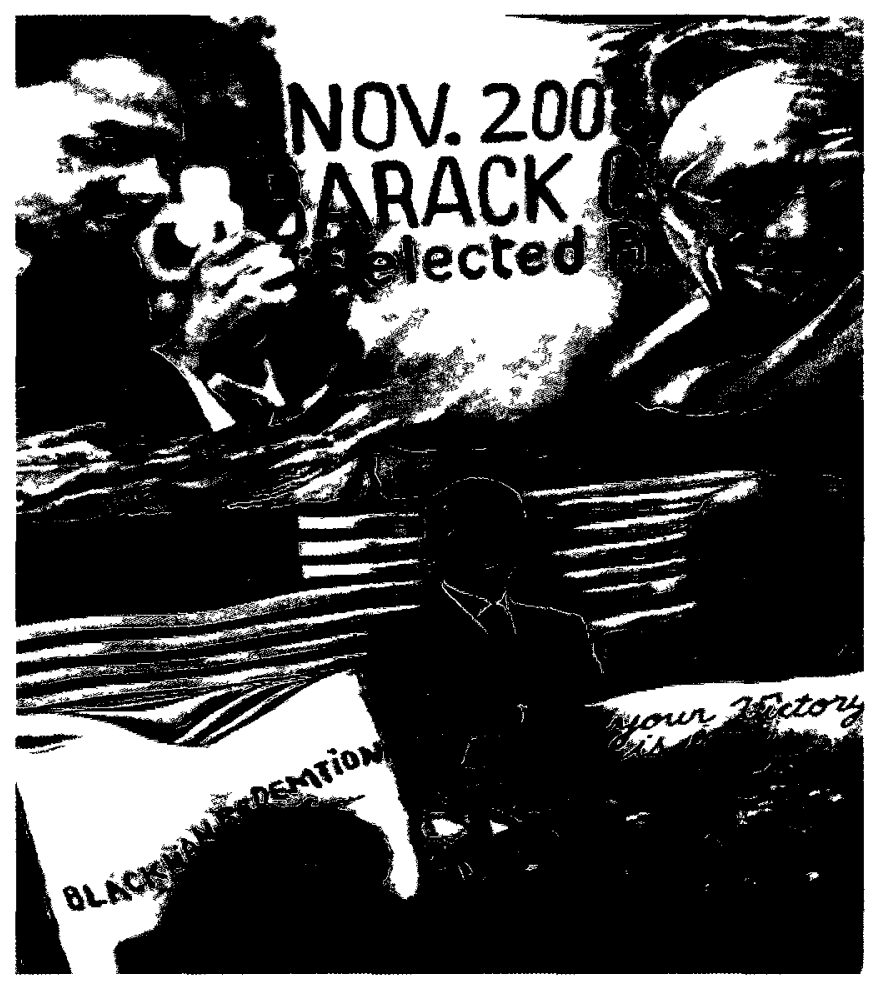

Fig. 6.3 - Mixed Media Collage by Haitian Artist Jean-Eddy Beauvoir. This panel is a sociopolitical piece where Jean has chosen to celebrate key African and African-American leaders from around the world. The theme of independence and freedom is very significant for Haitians since they were the first black-led republic in the world to gain independence with their successful slave rebellion in 1804.

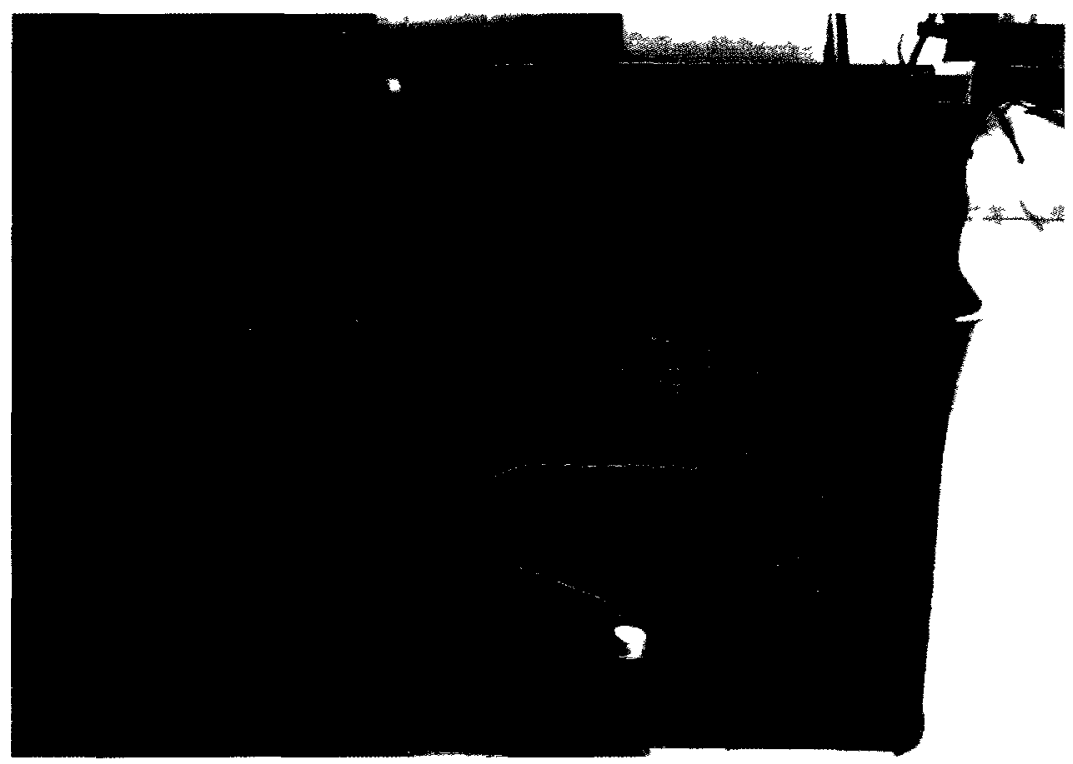

Fig. 6.4 - Mixed Media Collage by Haitian Artist Jean-Eddy Beauvoir - Another example of Jean's collage art again addressing the theme of independence and freedom similar to his piece above. 


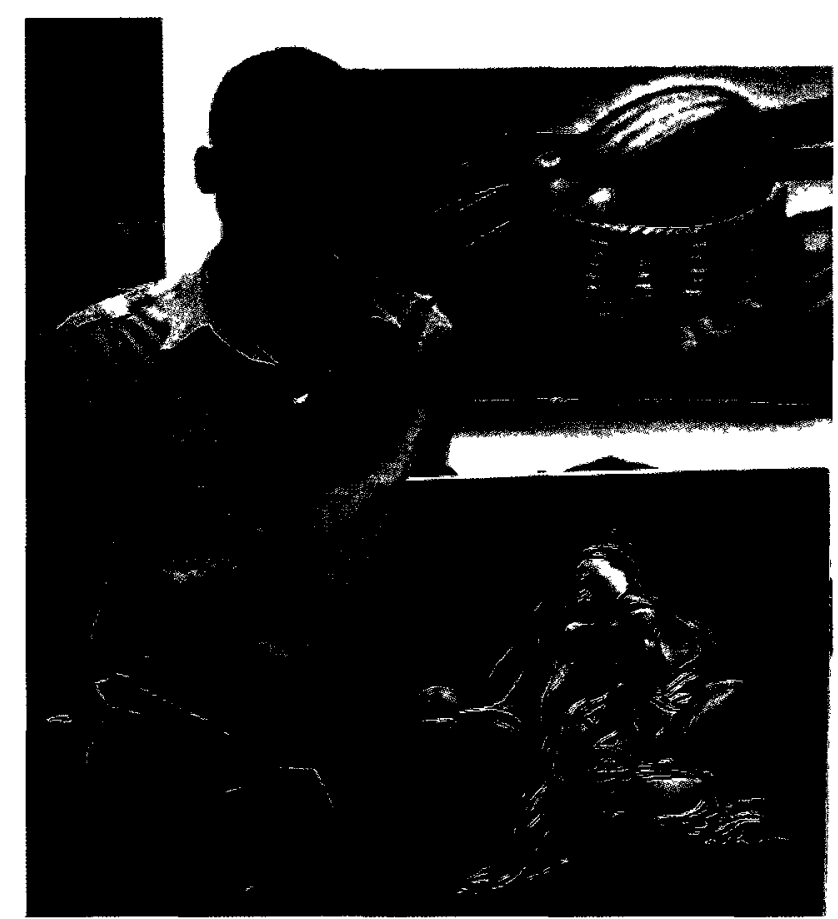

Fig. 6.5 - Acrylic Painting by Haitian Artist/Artist Assistant Ferdinand Madoche - This panel is celebrating the often neglected yet extremely important role of the woman figure within the Haitian society.

It is this very translation of cultural information direct from local people that begins to uncover genuine documentation to help create feasible ideas for the re-development of this community. Local knowledge obtained directly from the beneficiary is a powerful tool and the potential usefulness of this information began to reveal itself as the design workshop progressed.

As part of the March 2011 Rebuilding Léogâne Design Workshop, I was involved with the Human and Cultural Capital working group where I had the opportunity to meet with several local artists, sculptors and musicians. I learned many things from them but perhaps one of the more important was the sense that their culture is rich with art and artefacts that are made by the people and speak for the people.

The description and art shown below was constructed by a 28 -year- 
old local artist named Kenson Desire from Léogâne, Haiti. Desire was raised an only child by a mother that 'did everything for him,' which allowed him the opportunity to attend high school and pursue a university arts degree in Portau-Prince. He is now an arts teacher who specializes in calligraphy. He was given opportunities that the majority of Haitians never get and Desire utilizes his education to create meaningful cultural art pieces and teach younger generations the craft and significance of what he does.

"Everything that happens to me in my life goes into my work. So this is something that happened to me. In 2009, people were talking of how badly Rara was organized and structured. This made me think of all the problems that year. I said I will take the drums but it does not have the original form it should, the drum is broken. This represents the instability of our society during this period of time. The second piece is about a woman's pain. Because women are an important part of society but have suffered a lot at the hands of men. The head of this woman gives the sense that she has been abused by patriarchy and despite all the suffering she is still there. Haitian women despite all they have suffered, they are still strong". ${ }^{34}$

\footnotetext{
${ }^{34}$ Kenson Desire. Culture Group. Rebuilding Léogâne Workshop. (March 2011).
} 

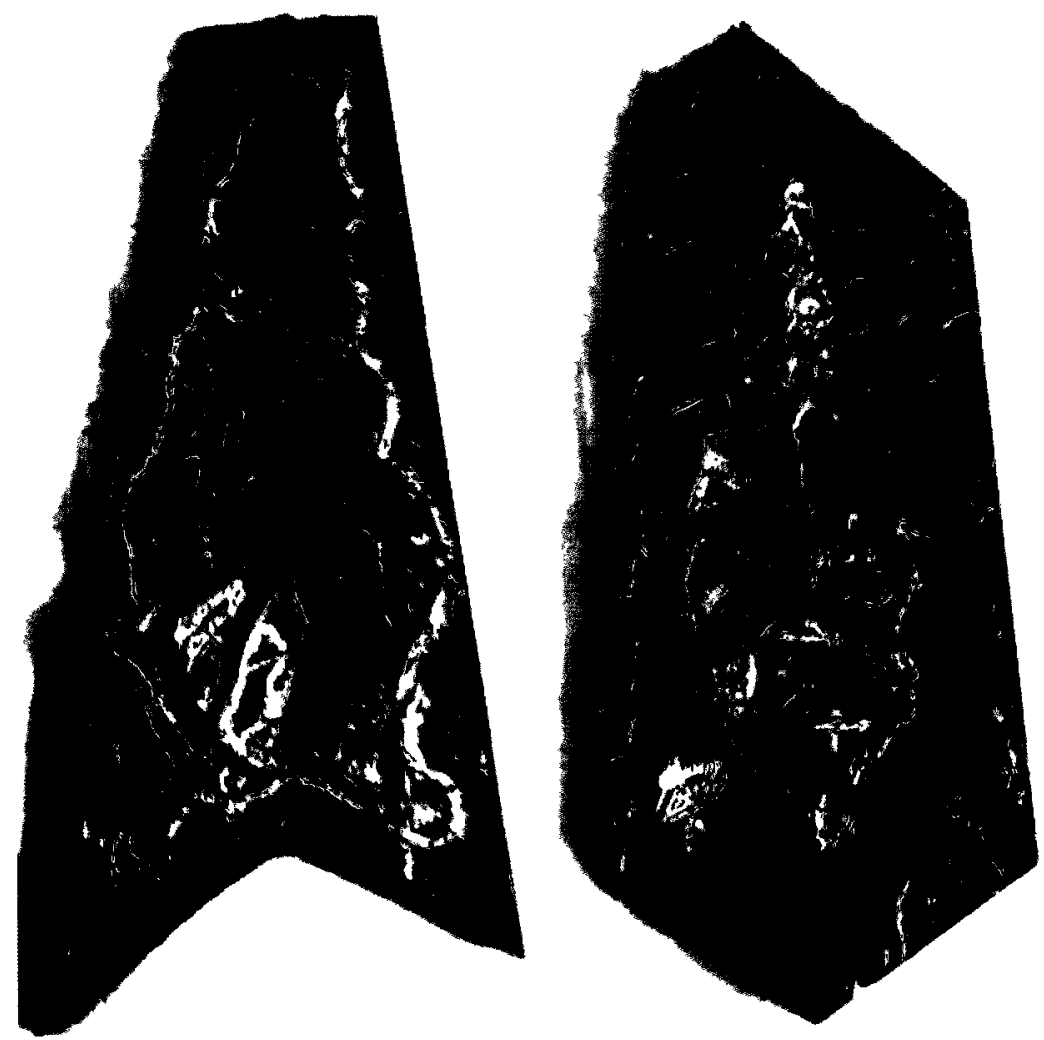

Fig. 6.6 - Clay wall hanging art (Arts Plastiques) - Crafted by local Haitian artist Kenson Desire from the small town of Léogâne.

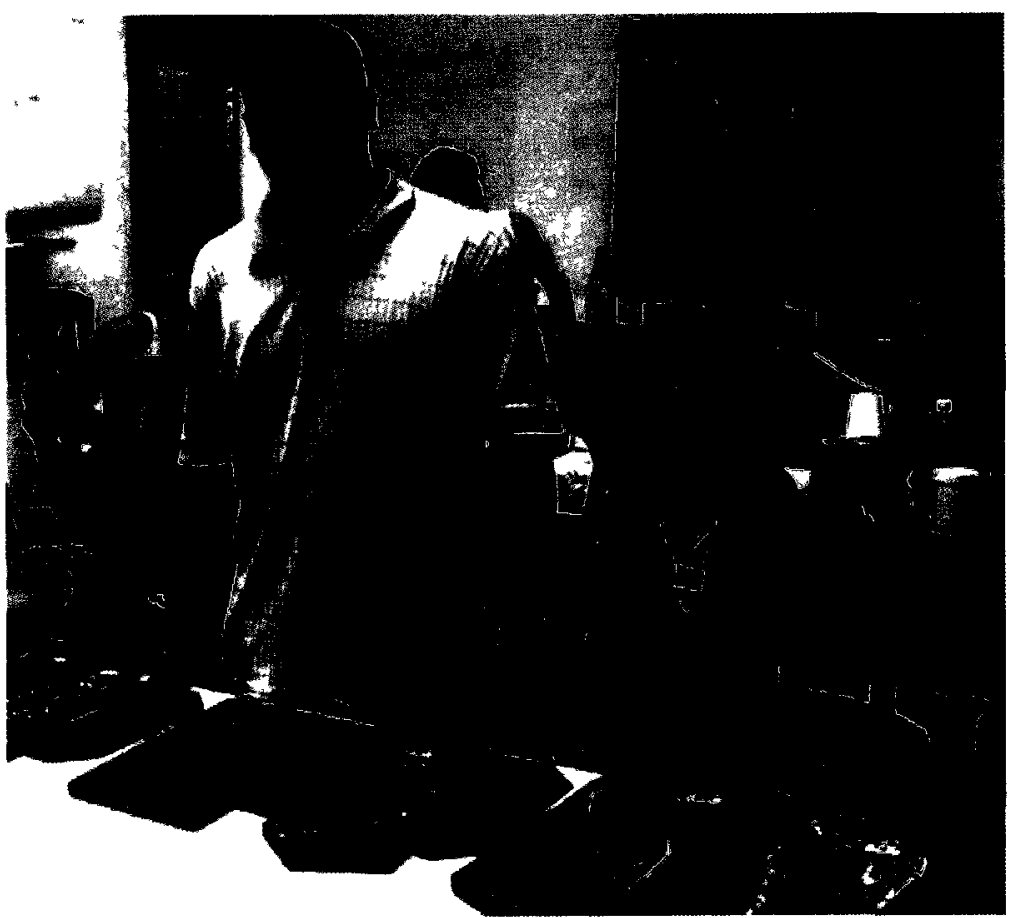

Fig. 6.7 - Kenson Desire displaying some of his art pieces at the Rebuilding Léogâne Design Workshop, March 2011. 
Interacting with local people gave me a chance to see the culture from a very authentic level. I learned that no matter how poor or underdeveloped the community, people would find ways to exist and operate amidst all the destruction and dire conditions. The majority of people did not have jobs but for the few that did generate income the expectation was they were the ones who provided for their family and friends; the sense of community was truly inspiring. I decided that my project would use this very sense of community and purpose to celebrate their diverse arts and music culture as a key component in initiating a sustainable long-term rebuilding strategy. 


\section{Project of Architecture: Post-Haiti Development}

Experiencing the Haitian culture firsthand has allowed me to better my understanding and formulate more informed opinions into the specific needs of this post-disaster community. Many of my suspicions were confirmed in the sense that designing with long-term subsistence in mind is a very involved process that takes careful co-ordination from many areas of expertise. Through the Rebuilding Léogâne Design Workshop I learned that the collection of local knowledge is invaluable and there is no substitute for such information. Perhaps most importantly though, I learned that even though rebuilding requires collaboration from many different groups the one form of information that will always be exclusive to the rebuilding community is the cultural and human capital.

Unlike a technical construction detail, a water and sanitation system, or the need for hospitals, schools and certain public services within similar developing nation scenarios, it is the culture that deals with the unpredictable social aspect of a society. It is the unpredictable nature of designing for postdisaster within the developing world that this research proposes as the catalyst for long-term subsistence and will develop into a project of architecture for the city of Léogâne.

Similar to the original proposed strategy in the hypothesis section of this research, I will use the same premise of a market square organization. The new name I have chosen to use for this project will be the Léogâne 
Cultural Exchange. The difference with the new direction is that instead of focusing of agriculture, Internet and mixed media the new Cultural Exchange project will be rooted in the local music and arts. It will be a place where citizens and artists from far and wide can gather, display, teach, perform, demonstrate or whatever their cultural art form may require.

Part of the problem in Léogâne is that people do not have enough reason to want to live and stay in this mid-sized town of approximately 40,000 people. "People want to do well so they can leave, there is very little to do and very little opportunity in Léogâne". ${ }^{35}$ Creating this cultural hub within the center of this community has enormous potential to attract tourism into the area and create more reasons for people to stay. The theme of connectivity begins to reveal itself as a key issue and can be explored in both the figurative and literal sense in relation to the rebuilding of Léogâne.

First there is the literal and physical connection to neighbours, which addresses the infrastructure in place such as electricity, sanitation systems, roads and public transportation. Second there is the figurative level of connection, which encompasses the desire and necessity to want to visit or live there. This research is laying foundation in 'reasons' to visit and live in this community. And with reason to visit comes people, which then translate into an increase in revenue flow leading to the eventual need for improved physical connections on a local, national and international platform. Being able to identify strengths and the relationships that may come out of using

${ }^{35}$ Culture Group. Notre Dame Haiti. (March 2011). 
them is an invaluable asset to any rebuilding project.

I have used a capacity and vulnerability analysis lens to identify the arts and cultural capital as a very strong capacity within Léogâne. Using this analytical tool to identify strengths allows rebuilding initiatives and my final project of architecture the ability to create a community specific rebuilding strategy that uses strengths to help transform community weaknesses into future strengths.

\section{capacities}

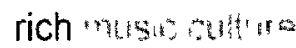
arts \& artisans existing Rara destination largest town in Leogane Commune

off of National highway rich sugar cane rum \& agriculture potentials diverse and rich culture, 10-

cal \& national history many people looking for work

port town with appealing waterfront $\&$ beaches

\section{vulnerabilities}

little reason to visit Leogane

lack of jobs

poor housing/education \& civil services

little reason to $5 t 0,3 \cdots, E$ in

Leogane

excess rubble in streets lost historical \& cultural information unorganized rebulding efforts

- poor town infrastructure lack of overall vision

Fig. 7.1- Léogâne Capacities \& Vulnerabilities diagram - an example of some existing strengths and weaknesses within the community. The purpose of this image is to identify relationships between the two sides so rebuilding initiatives can better align themselves to target key issues. This allows capacities the opportunity to transform vulnerabilities into community strengths over time.

\section{Site Selection}

The site I have chosen is geographically located at the centre of Léogâne and is the location of the Old Town market square. 


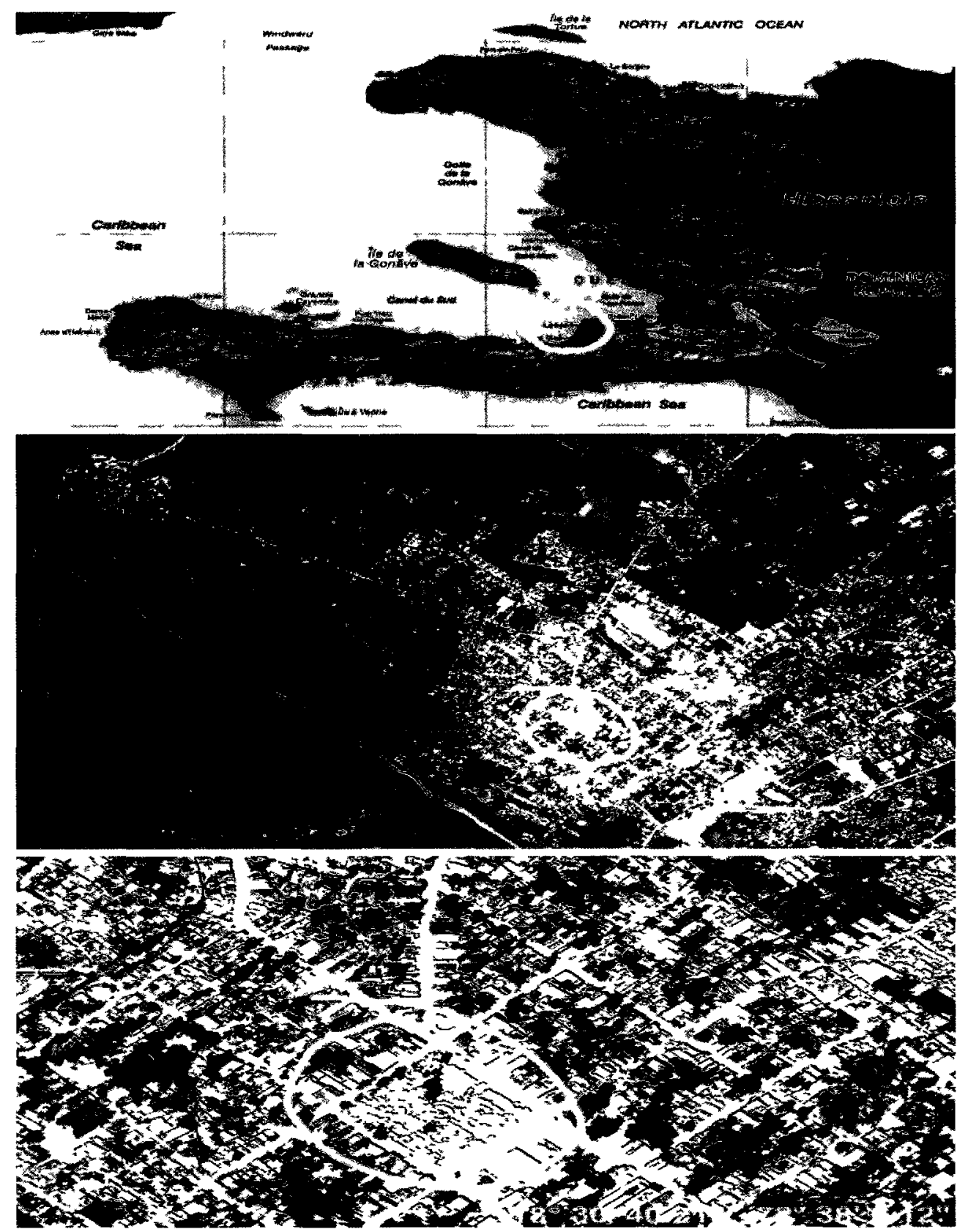

Fig. 7.2 - Frame of reference site photos - Haitı natıonal map, community of Léogâne aerıal photograph, market square downtown Léogâne aerıal photograph

This city market square also holds historical importance since it comprises the remaıns of a once colonial settlement surrounded by bits and pieces of historic bulldings. To the south east of the market site is the recently re- 
constructed Saint Rose of Lima Catholic church that was destroyed in the 2010 earthquake just one year before it was to turn five hundred years old, an old city hall building to the north and many others once important fragments of colonial architecture and city plan.

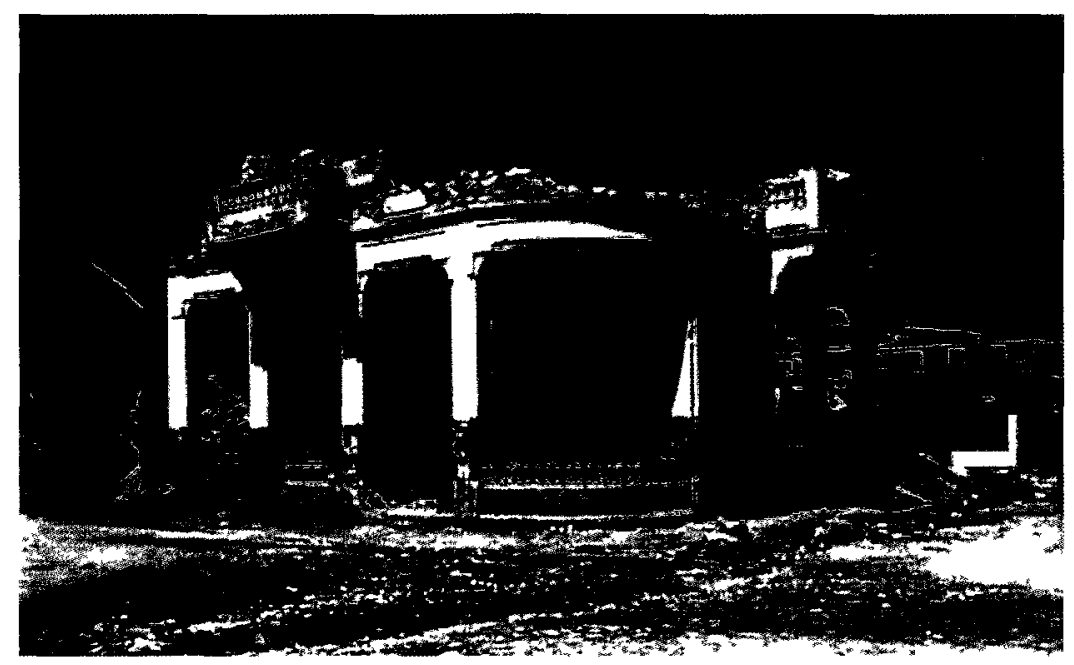

Fig. 7.3 - Historic colonial public building March 2011, Léogâne Haiti. The bottom floor of this building was used by artist Jean-Eddy Beauvoir before being destroyed in the earthquake. Located on the southwest corner of Rue de L'hopital and Rue La Croix facing the Old Town market square.

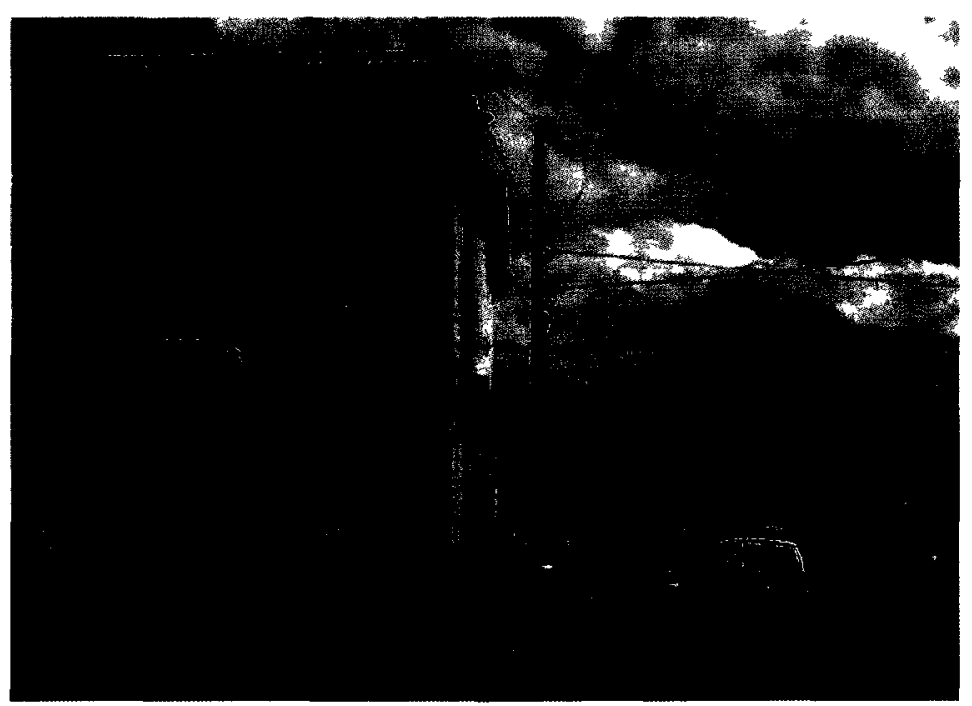

Fig. 7.4 - Historic old city hall building March 2011, Léogâne, Haiti. Located on the northeast corner of Rue St. Yves and Grand Rue facing the Old Town market square.

The selected site is currently being occupied by a mixture of squatters 
and market vendors who have created temporary shelters and market stalls after the Jan. 12, 2010 earthquake. At the Rebuilding Léogâne Workshop I was informed that the "tent cities have been decreasing with time"36 as rubble is removed and people's homes are rebuilt and safe to return.

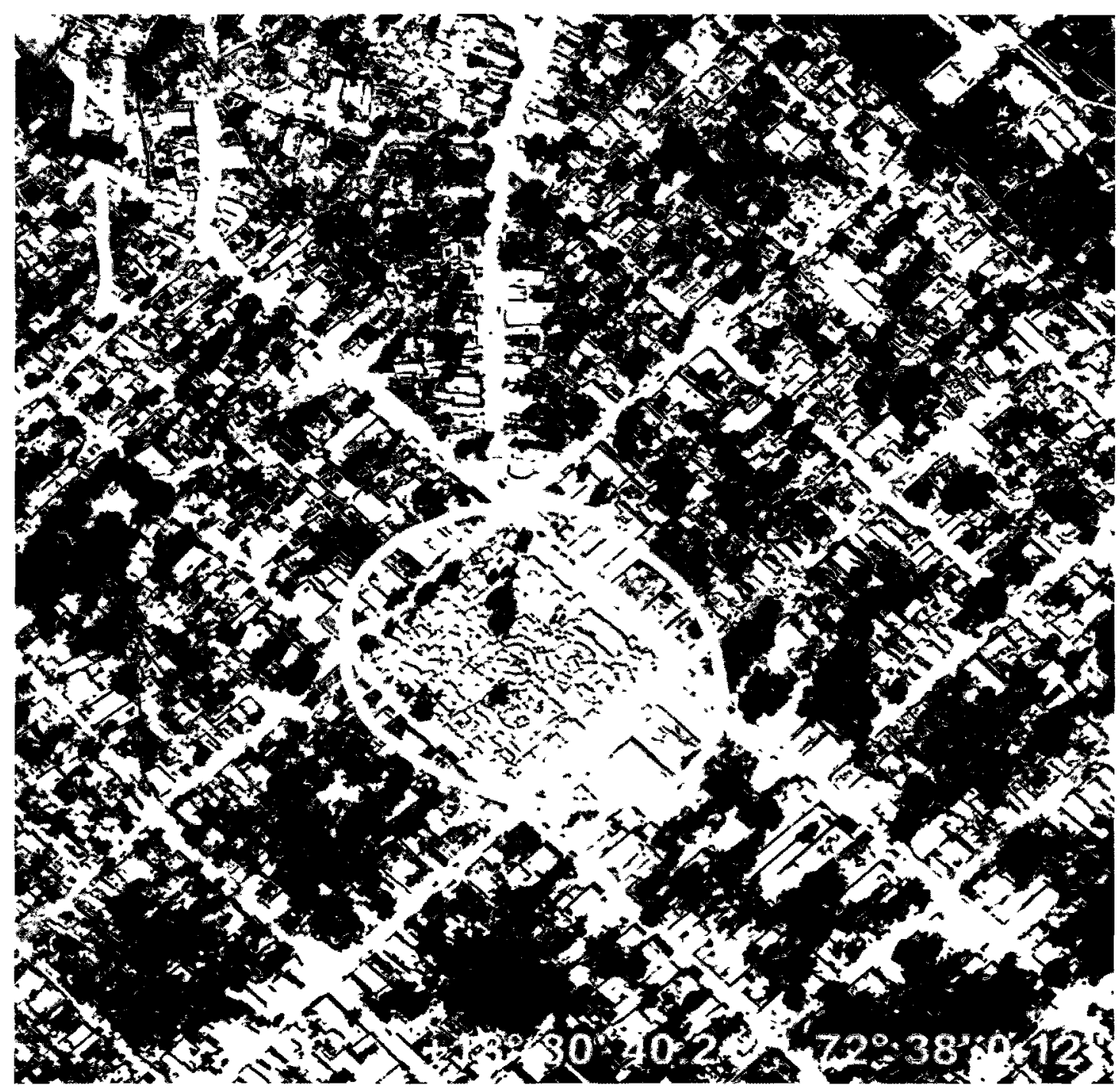

Fig. 7.5 - Aerial photograph of the project site - Downtown Léogâne, Haiti at GPS coordinates $+18^{\circ} 30^{\prime} 40.21^{\prime \prime},-72^{\circ} 38^{\prime} 0.12^{\prime \prime}$.

${ }^{36}$ Culture Group. Notre Dame Haiti. (March 2011). 


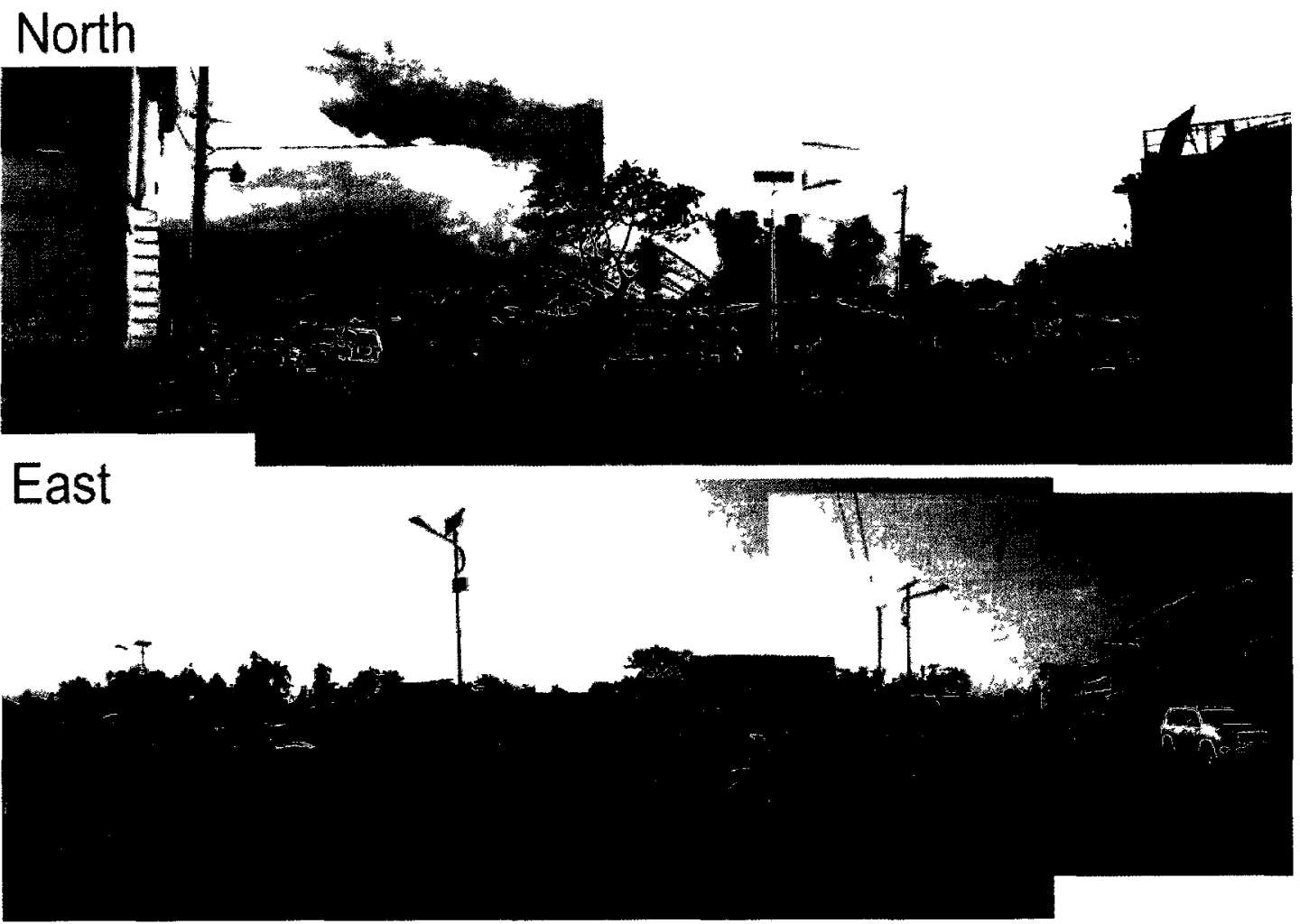

South
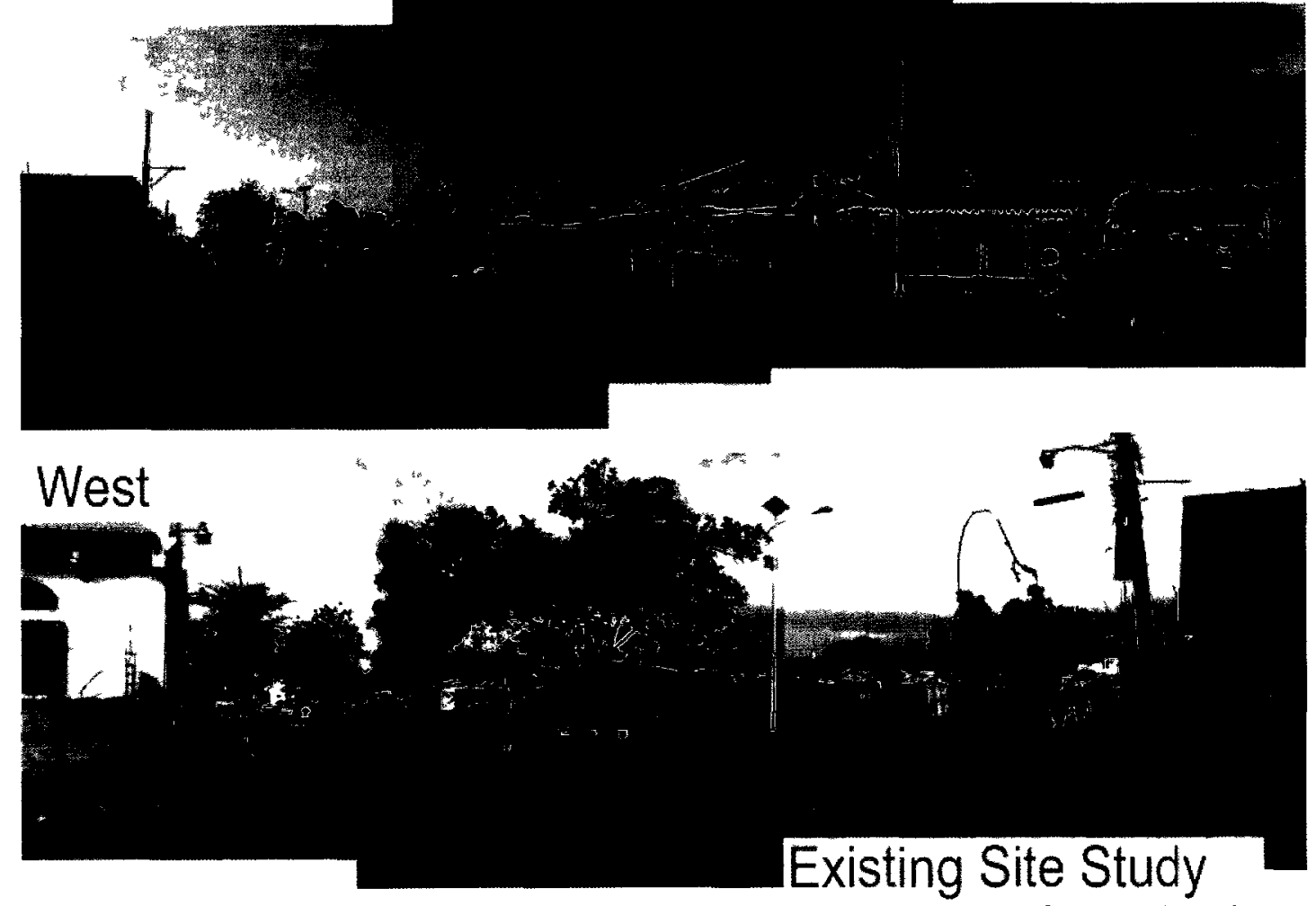

Fig. 7.6 - Existing site documentation panel. Views of the site conditions from each major intersection surrounding the town square site. 
Being involved in the rebuilding workshop was an invaluable tool that allowed me to discern the general organization of the town and how it functioned pre and post-earthquake. I learned many interesting and random pieces of information about the town's culture directly from the local people.

I learned, for example, that during Rara season (Haitian music celebration) companies will sponsor and erect bleachers along the main roads to use as their own public or private seating for the event. I learned that the rum being distilled near the Léogâne port is causing water pollution and undesirable swimming conditions. I was also informed about the role of 'market women' within the local culture and how it is the women's responsibility to grow, transport and sell agriculture at the market. Women will either walk or arrange transportation down from the mountains where much of their crops are typically grown in order to sell produce at the Léogâne and Port au Prince markets. I did learn about one exception to women's handling of agriculture though, and that is when it came to sugarcane this was a man's business only, and the men would be in charge of growing and selling it to distilleries to make sugarcane $\mathrm{rum}^{37}$

Although not all of this information directly translates into Léogâne's architecture and geography, it painted a broader picture of what the community is about, the people who live there and the types of normalities that occur on a daily basis.

\footnotetext{
${ }^{37}$ Culture Group. Notre Dame Haiti. (March 2011).
} 


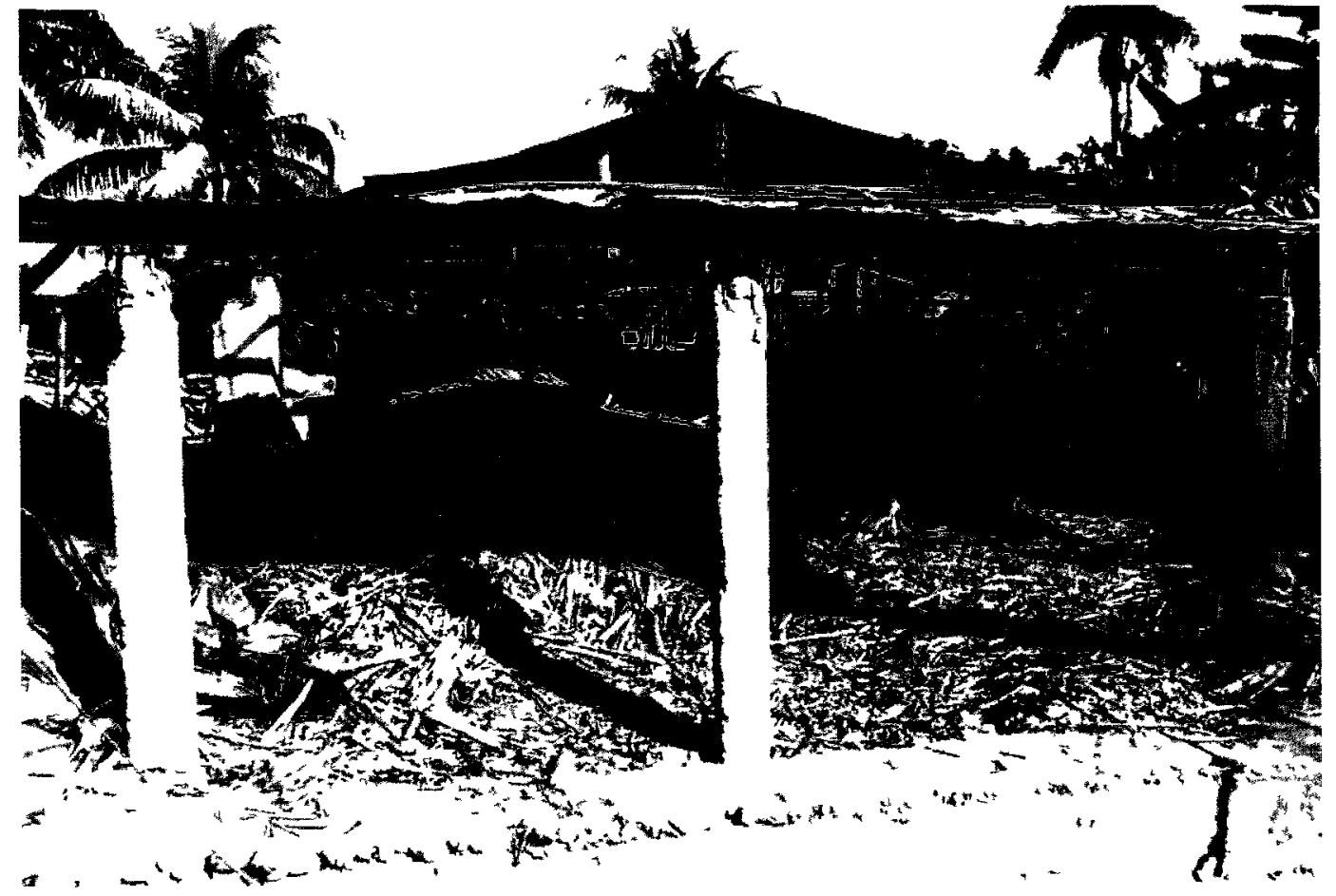

Fig. 7.7 -Sugarcane rum distillery, downtown Léogâne, Haıtı

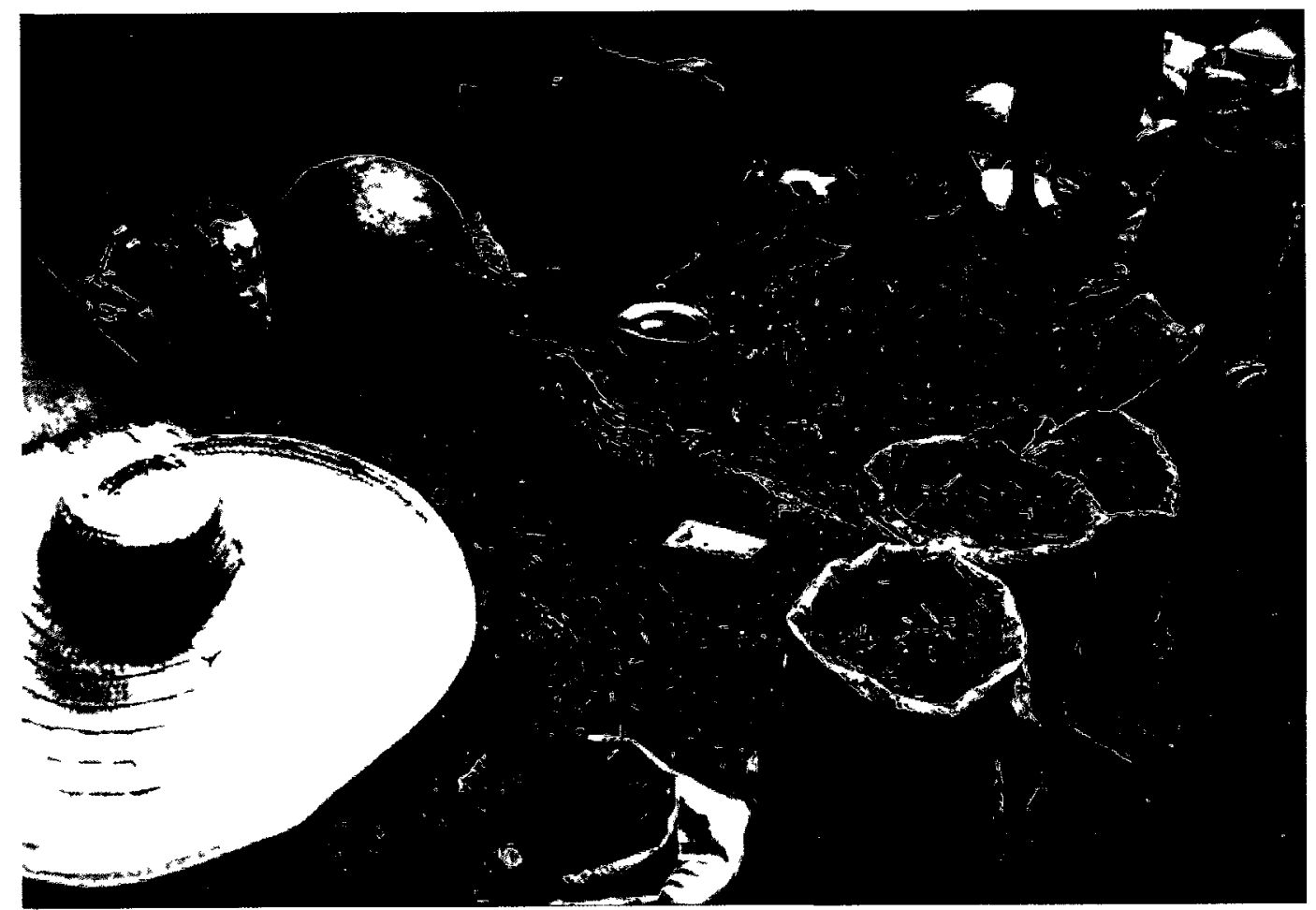

Fig. 7.8 - 'Market Woman'. Local market in Léogâne where charcoal is being sold. Charcoal is used as a primary fuel source for cookıng in the community. 
Other important site-specific issues that have not yet been addressed in this text but will be in the final design include: utilizing the recently installed solar light sources around the perimeter of the market square; preserving all mature trees on the site; respecting heights and historic building periphery; and respecting the relocation of existing market vendors within the new design.

\section{Building Materials Research \& Project Strategy}

The material strategy I have chosen to use for my project will be a traditional material palette that is typically being used and proposed for the majority of new construction projects in the area. The building materials will include concrete block for permanent structures, steel for framing and miscellaneous structural components, corrugated metal roofing materials and woven dried banana leaf panels for partition walls.

It is difficult to address local building materials in Haiti without first discussing the shortage of lumber due to deforestation within the country. "In 1923 , over $60 \%$ of Haiti's land was forested; by 2006 , less than $2 \%$ was" ${ }^{38}$ It did not take long to transform a readily available building material to a scarce natural resource. This can be attributed to over demand and lack of policy in regards to clear-cutting and replanting regulations.

"Beginning in about 1954, concessionaires stepped up their logging operations, in response to Port-au-Prince's intensified demand for charcoal,

\footnotetext{
${ }^{38}$ Library of Congress, Federal Research Division. Haiti Country Profile. <lcweb2.loc.gov /frd/cs/profiles/Haiti.pdf>. (May 2006) p.10.
} 
thus accelerating deforestation, which had already become a problem because of environmentally unsound agricultural practices, rapid population growth, and increased competition over scarce land", ${ }^{39}$

As result, this once traditional building material is no longer an economically viable option for new building projects. Instead, the use of steel and cementitious building elements are the materials of choice that are both accepted by locals and a more economically obtainable option.

"While CMU posed definite vulnerabilities in terms of material quality control and poor installation techniques, it has positive attributes in the eyes of the community that need to be recognized in the downtown in terms of security and familiarity especially with mixed-use stores" ${ }^{40}$

Other important issues in regards to material selection are acceptability within the project community, safety concerns and quality control. Selecting vernacular materials from the community not only adds a layer of familiarity to the project, but also provides local industry and trades persons the immediate opportunity to participate and lend their expertise and labour to the project.

In terms of material connections and detailing for the new Cultural Market, a simple and locally inspired approach will be used for these details. Motivated by the makeshift building culture that exists within the community of Léogâne, the Cultural Market will use modest material connections that celebrate and use the properties of the material to achieve desired results.

\footnotetext{
${ }^{39}$ Library of Congress, Federal Research Division. Haiti Country Profile. <http://www.lcweb2.loc.gov/cgibin/query/r?frd/cstdy: @ field\%28DOCID+ht0064\%29>. (Dec.1989).

${ }^{40}$ Rebuilding Léogâne, Housing Group. Notre Dame Research Facility Haiti. (March 2011).
} 


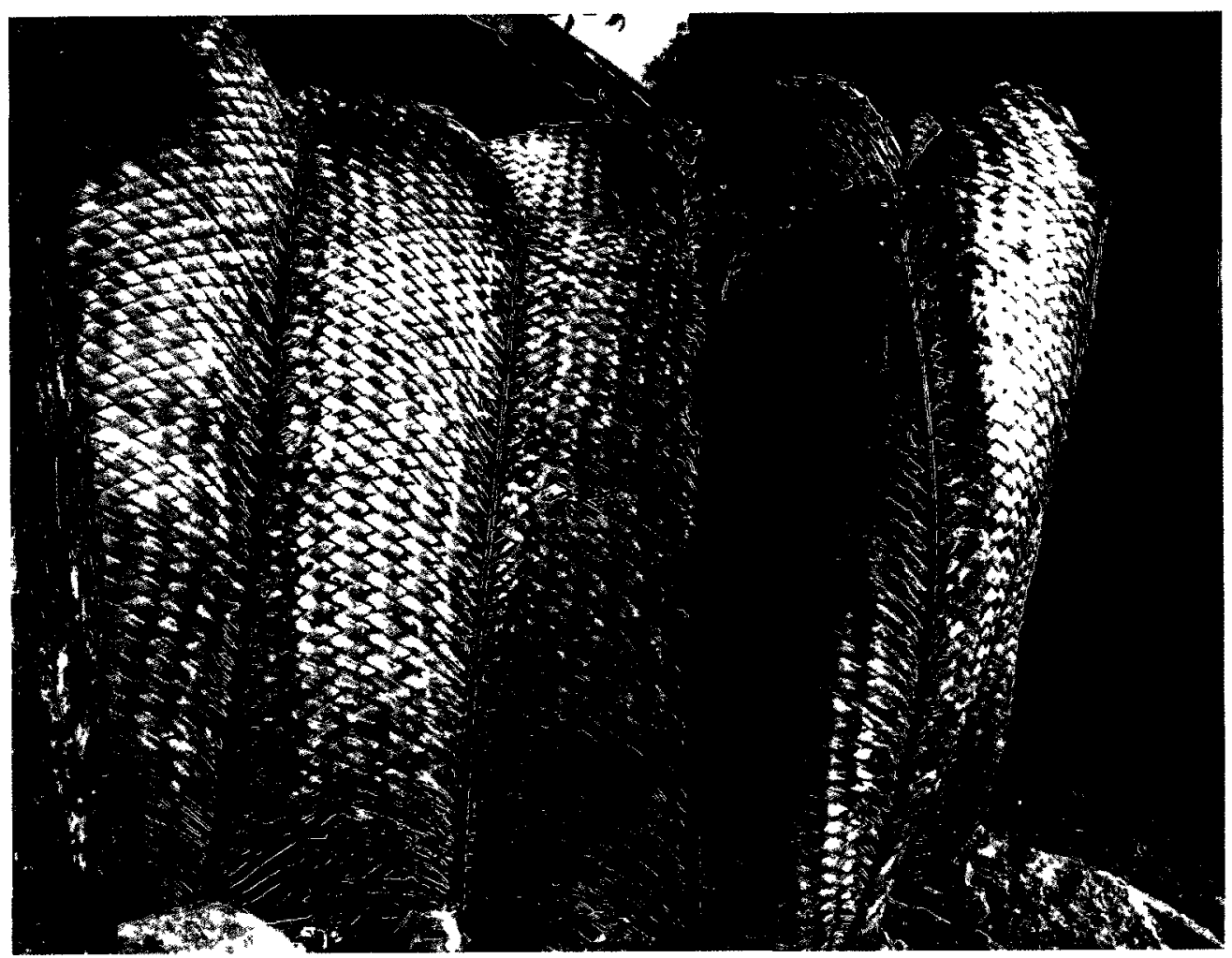

Fig. 7.9 - Dried banana leaf partition wall panel, Léogâne, Haiti 2011.

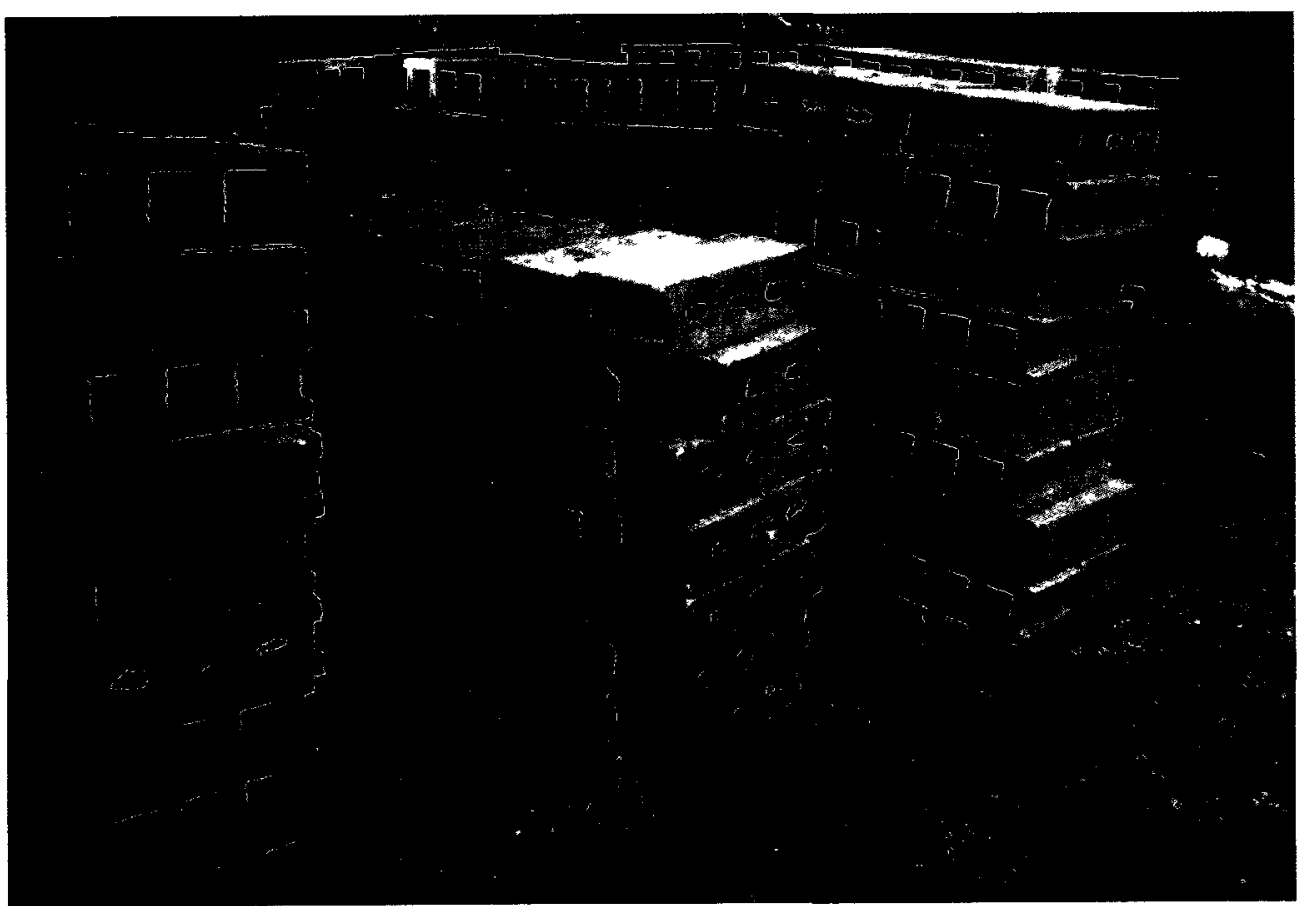

Fig. 7.10 - Concrete blocks stacked at a local construction site in Léogâne, Haiti 2011. 


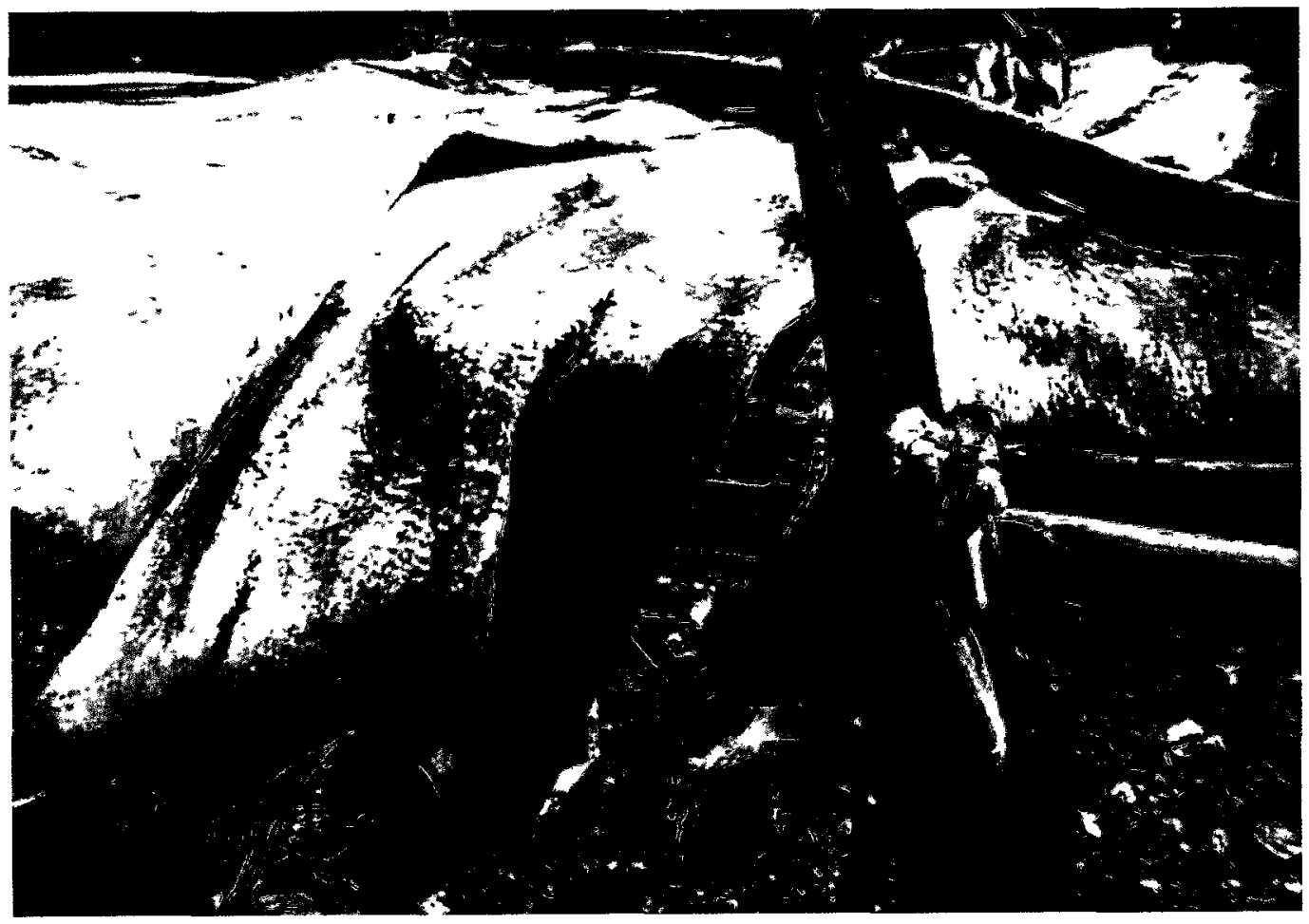

Fig. 7.11 - Local material connection. Bamboo uprights on a small cooking shelter are fastened together with a dried banana leaf twine. Léogâne, Haiti 2011.

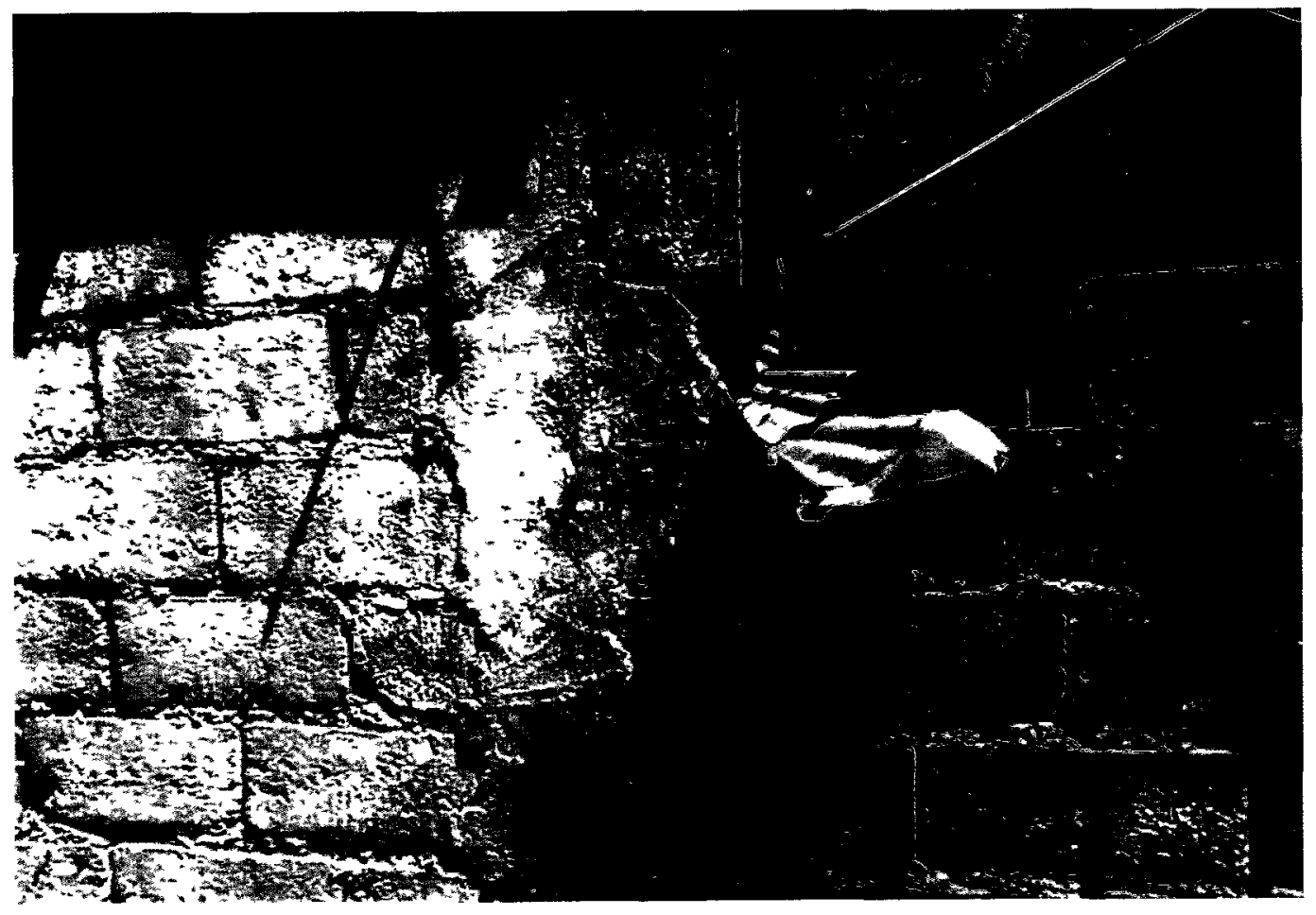

Fig. 7.12 - Makeshift eaves trough detail. Léogâne, Haiti 2011. 


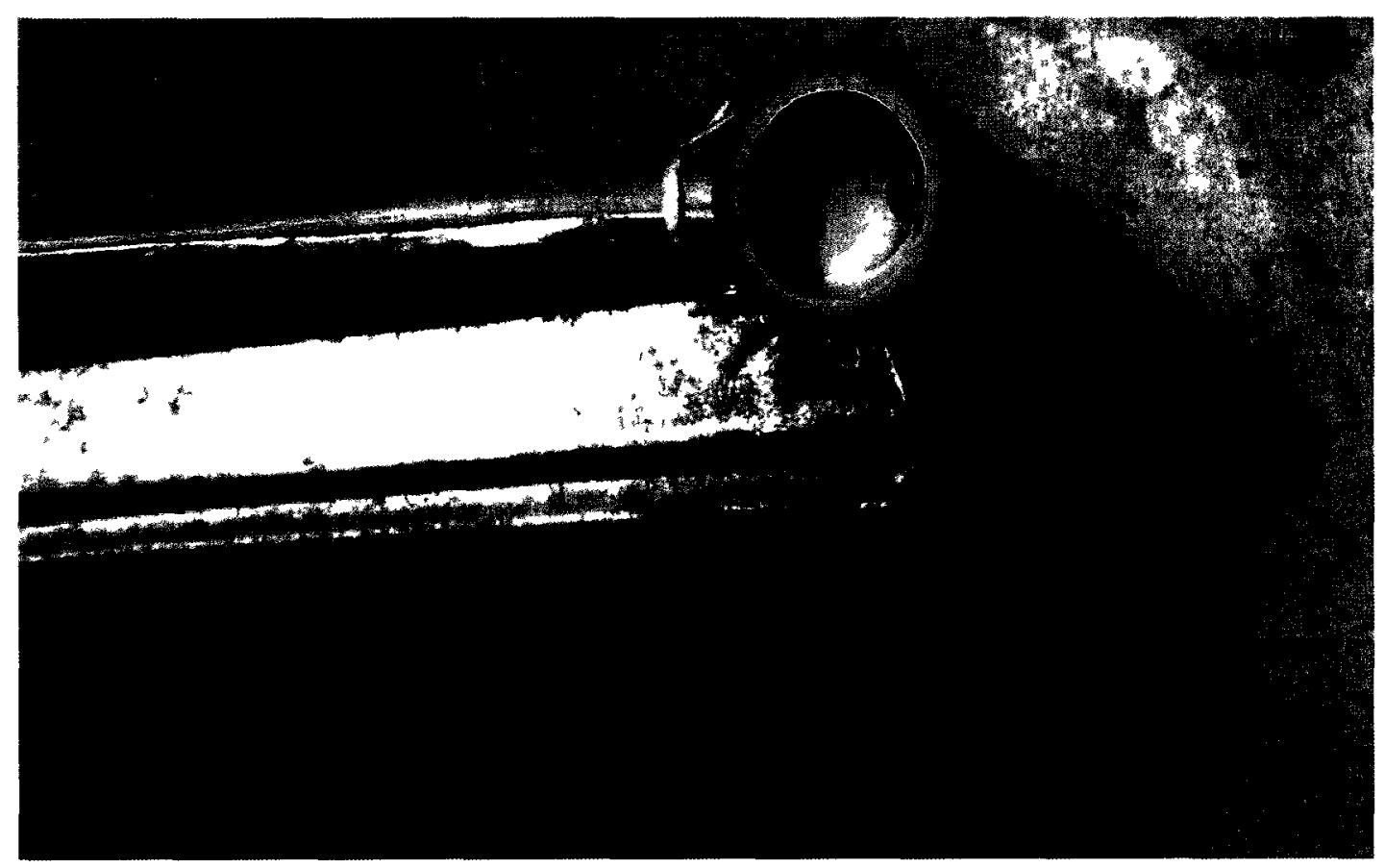

Fig. 7.13 - Bamboo and metal roofing construction detail. This model is a locally inspired connection that investigates a tectonic option for the temporary market stalls as part of this proposed project of architecture: Léogâne Cultural Market.

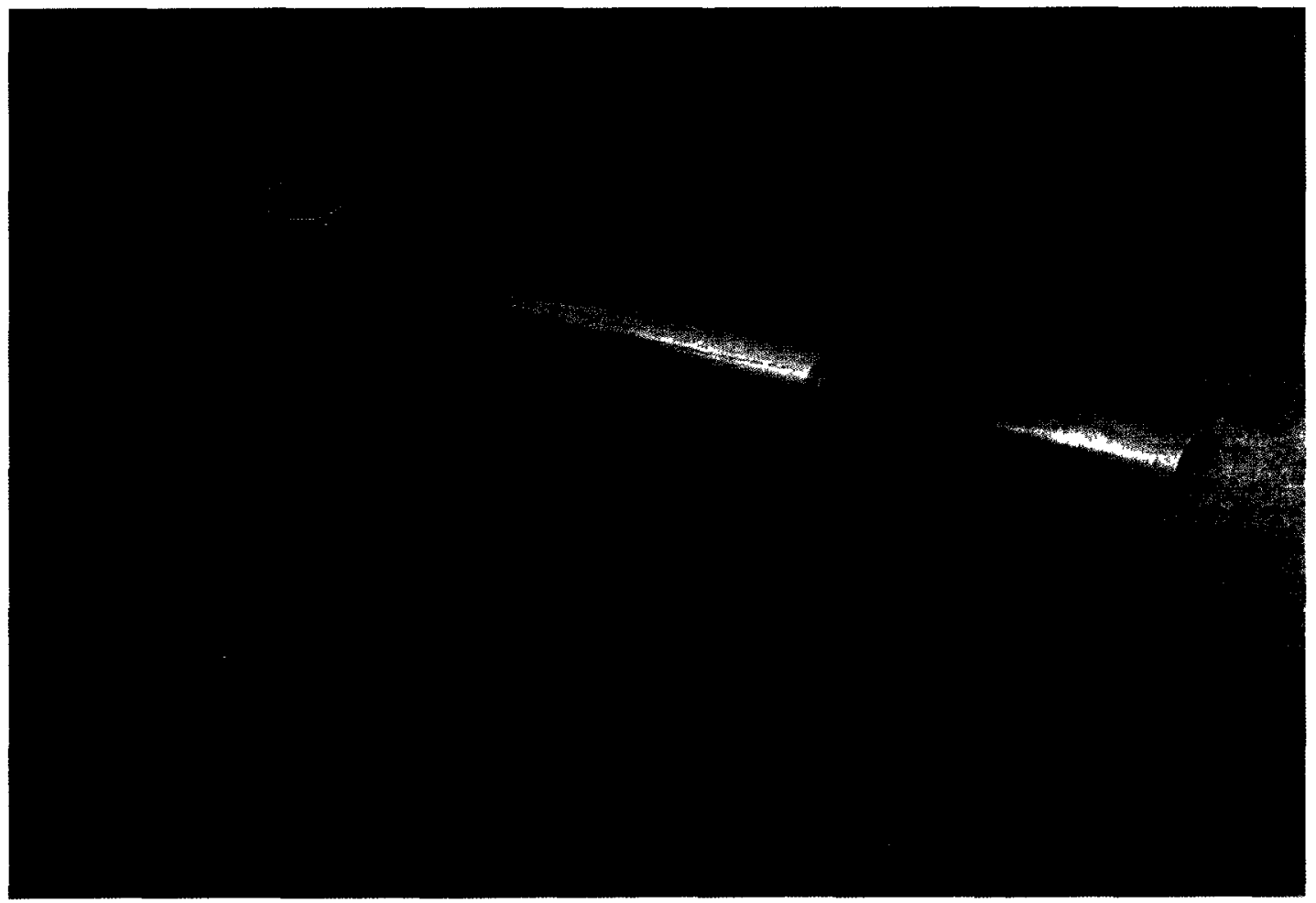

Fig. 7.14 - Bamboo construction detail. Another locally inspired connection as a proposed strategy for the temporary market stalls. 


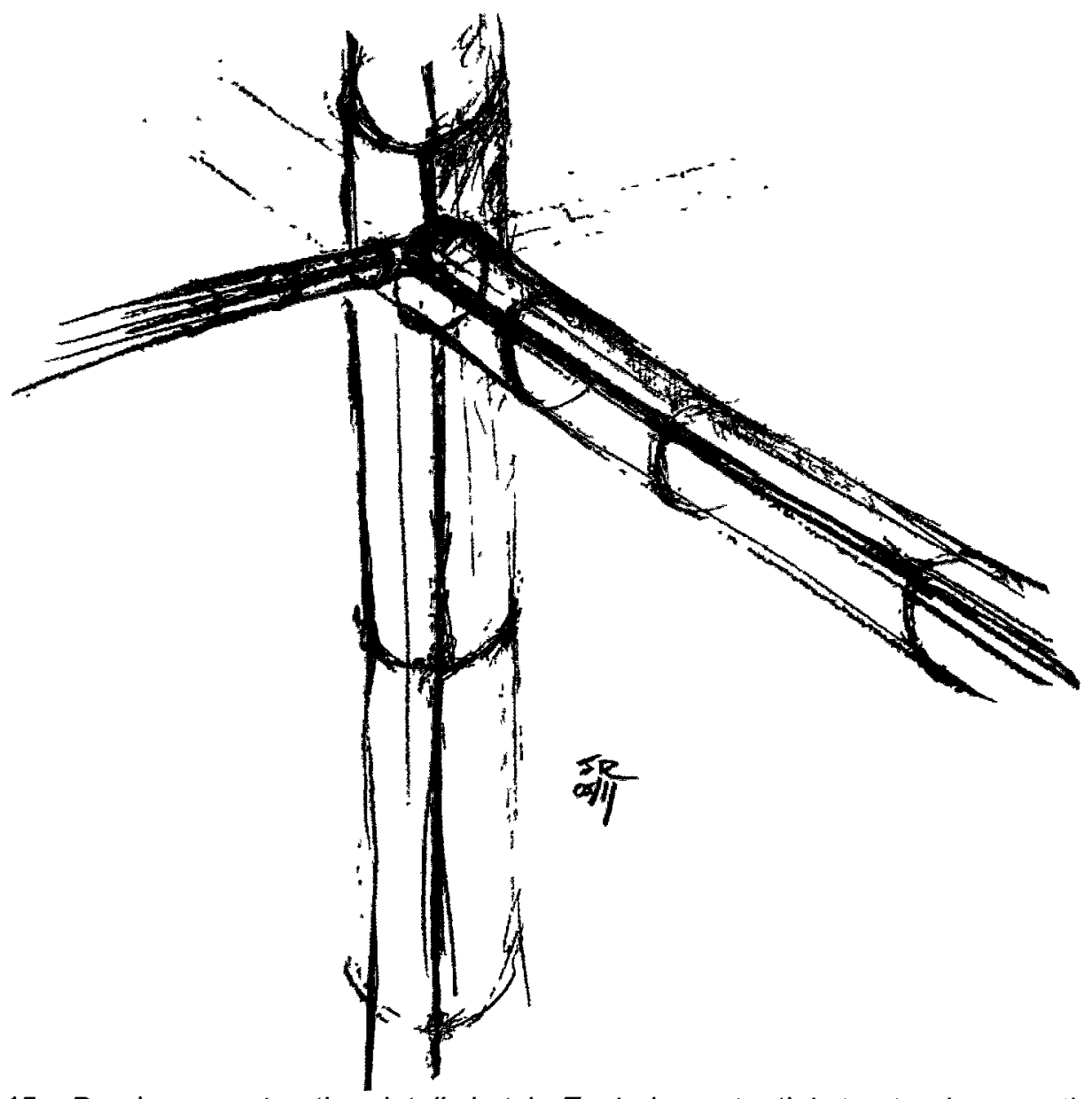

Fig. 7.15 - Bamboo construction detail sketch. Exploring potential structural connections for both temporary and permanent construction projects.

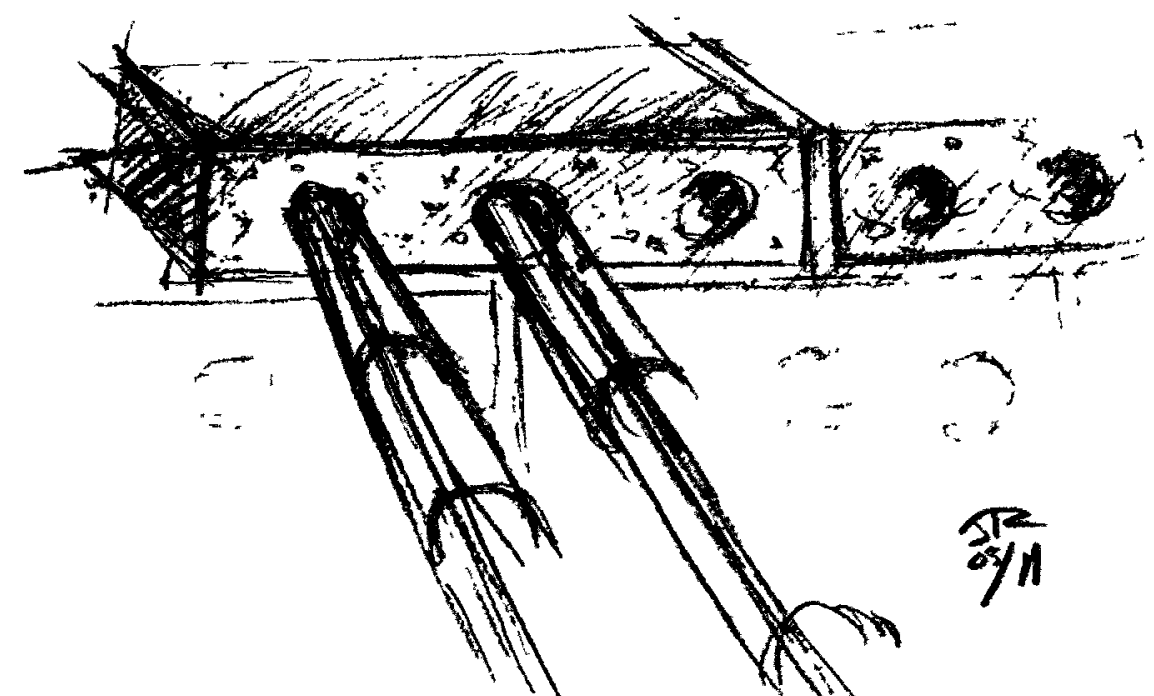

Fig. 7.16 - Concrete block to bamboo connection. This sketch investigates an alternative method to provide lightweight structural roofing for small scale construction projects. 


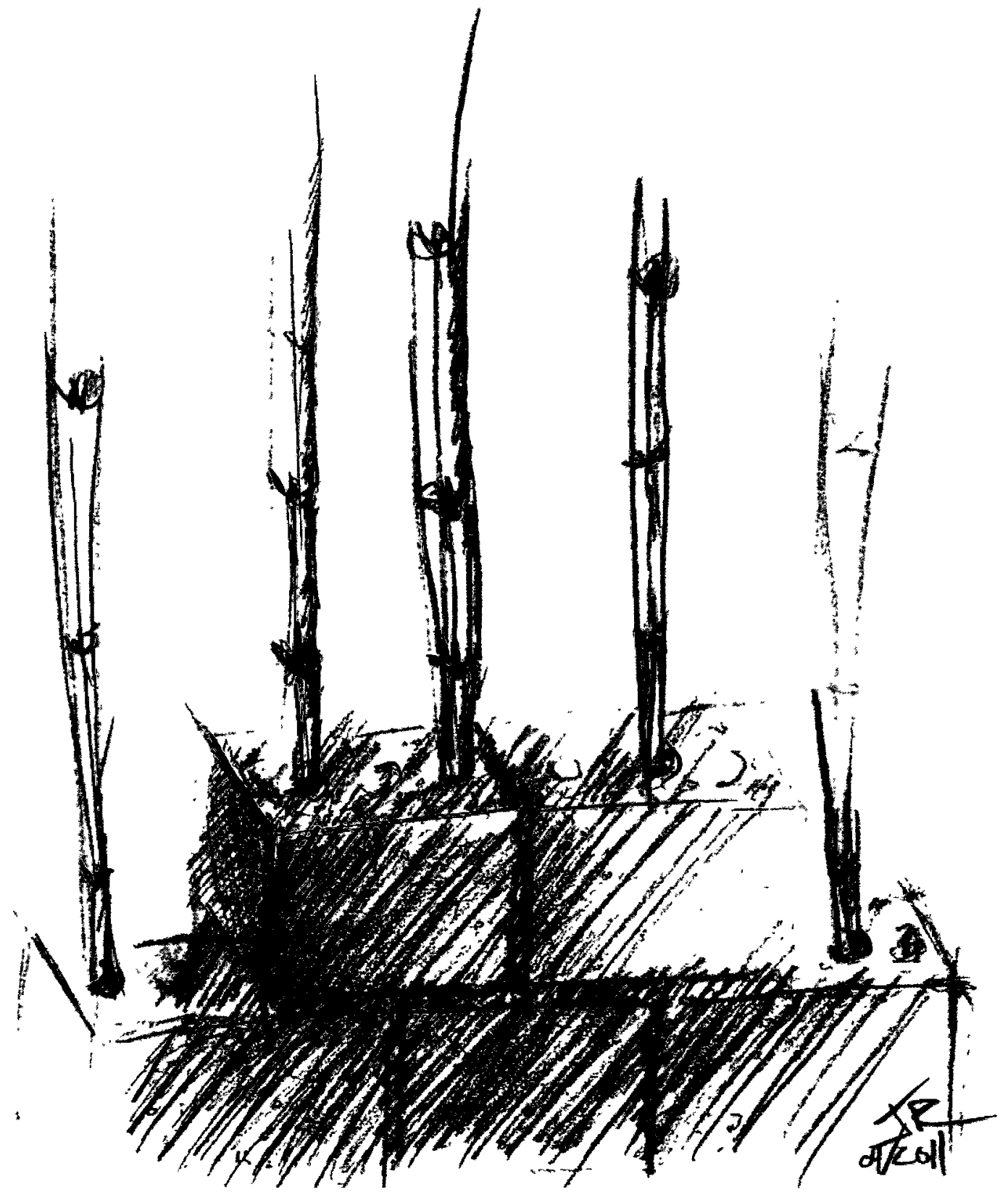

Fig. 7.17 - Concrete block to bamboo connection II. This sketch explores bamboo as a form of partition wall and structural component extending its self from a concrete block wall.

Cultural Market Program

The new Cultural Market will be a mixed-use project that introduces infrastructure for the arts into Léogâne, which will ultimately be a conduit to 
display, teach and celebrate their culture. The project site is roughly divided into four quadrants with a centralized amphitheatre component for people to gather. The organization of the site plan was derived from the main intersections located to the north, east, south and west corners of where the majority of pedestrian flow will be coming. The celebration of Rara also played an integral role in determining a general site strategy that would cater to bands and followers to approach and enter the site from all directions and then be drawn into a centralized amphitheatre area.

The program of the new Cultural Market is as follows:

- Amphitheatre (gathering area, performing stage, sports \& recreation)

- Storage and changing facility (event storage, latrines, change rooms)

- Classroom and teaching area (quiet area to practice and teach)

- Permanent artist studio stalls (located at perimeter of square)

- Water pumps and rainwater collection (non-potable water source)

- Public art installation (convey the spirit of the city)

- Temporary market stalls (modular/removable stalls)

The general organization of the site plan design can be broken down into three general 'layers of program,' all of which radiate out from the central amphitheatre. 


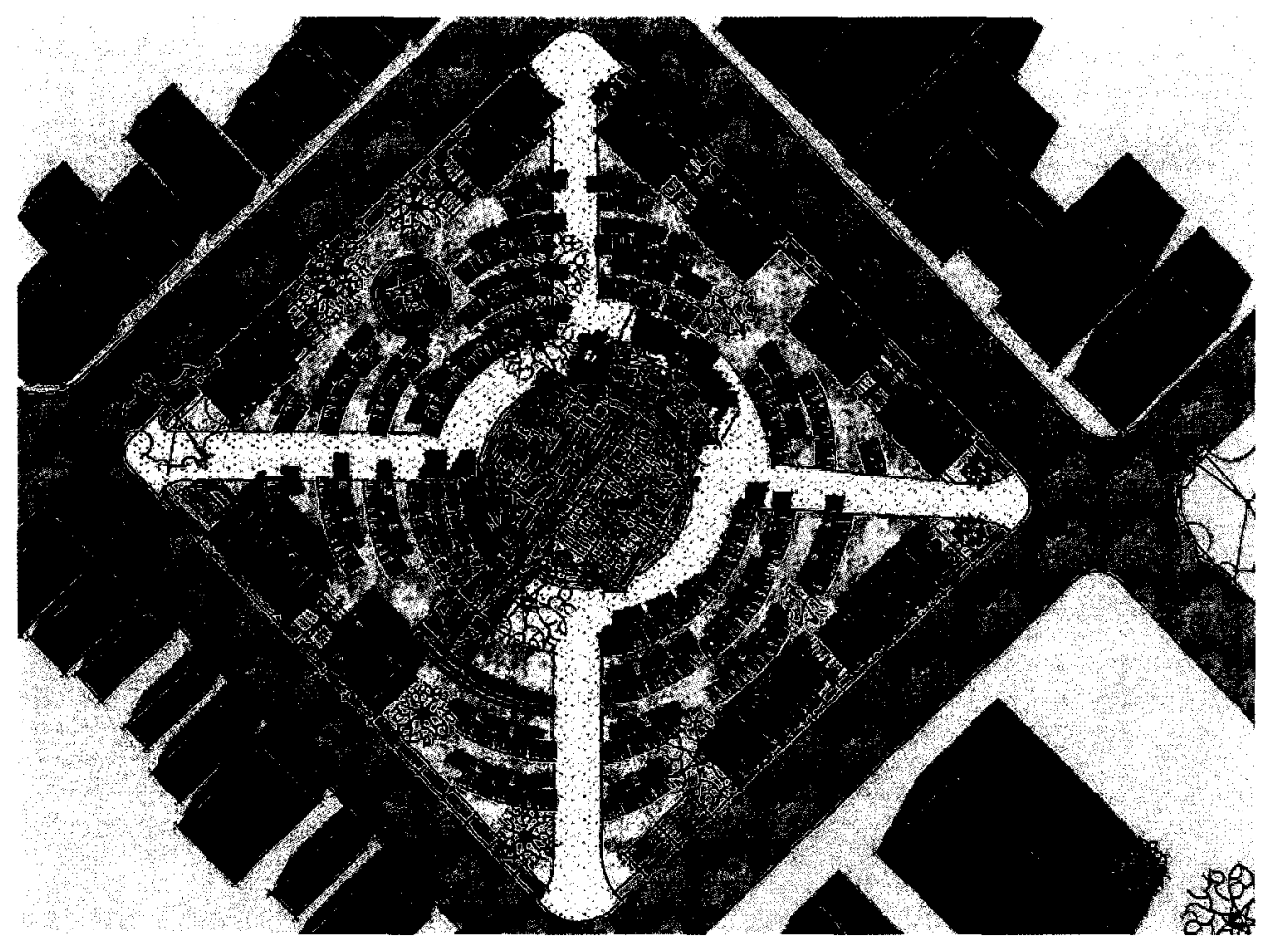

Fig. 7.18 - Cultural Market Master Plan. Depicting full occupancy of temporary market stalls located in circular formation radiating out from the central amphitheatre. Léogâne, Haiti.

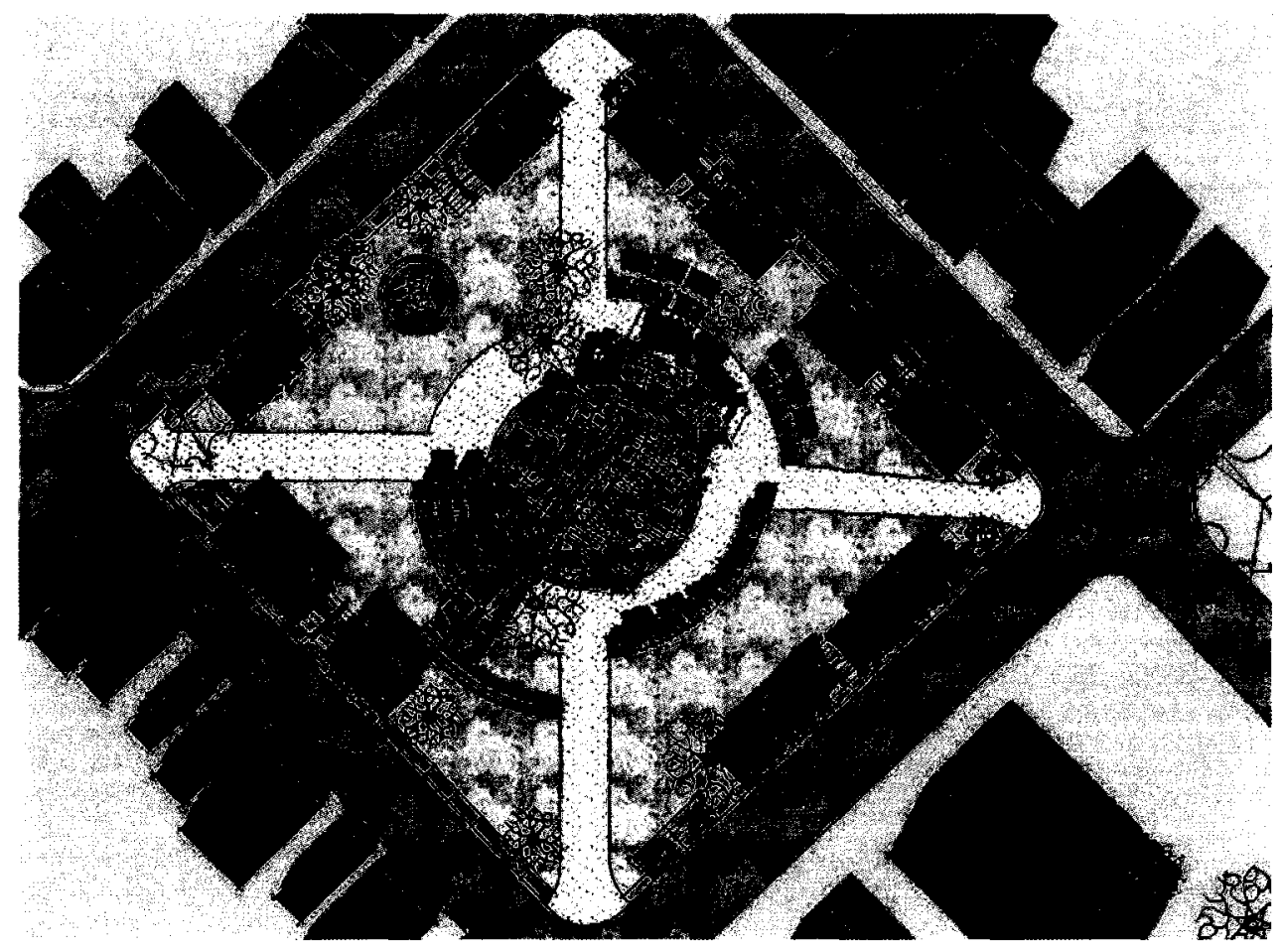

Fig. 7.19 - Cultural Market Master Plan II. Depicting partial occupancy of temporary market stalls allowing for more public space making room for festivals and events. Léogâne, Haiti. 
Located around the outermost layer are three primary elements: water collection and rainwater run-off stations, permanent artist studio stalls, and built-in bleachers. The main component of these is the permanent market stalls which currently exist, albeit in much more of a make-shift fashion. The new market stalls will be designed specifically for artists to use as multi-purpose spaces where they can work, practice, teach and display work within the same area. The introduction of steel and CMU built stalls will also allow people a safe and secure place to display and store their materials and products.

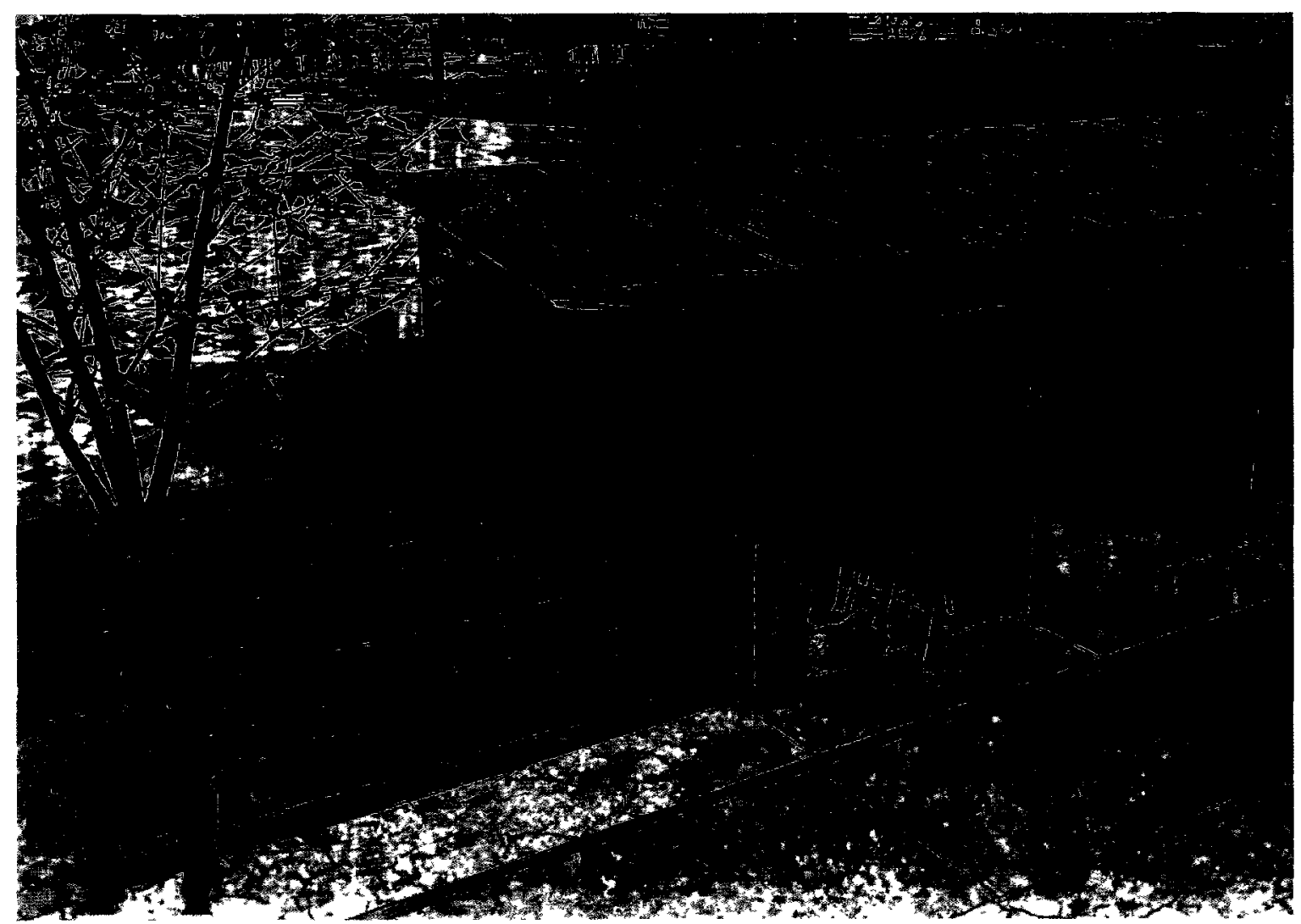

Fig. 7.20 - Cultural Market permanent artist stalls and festival bleachers. Léogâne, Haiti. 
Situated between the artist stalls will be rainwater run-off troughs and water pump stations. This is a community connection component that will allow for casual interaction while collecting water and add another layer of vulnerability reduction in that it creates a readily available and reliable water source. Incorporated into the design of the water stations will be public festival bleachers built in as a continuation of the wide sidewalks and streets. This area will not only provide seating for cultural festivals such as Rara, but also act as a form of park bench for larger groups, a place for the community to gather and also act as an outdoor informal teaching area for the artists and artisans.

In contrast to the outer layer of permanent program on the Cultural Market site is the middle layer of program which incorporates the use of temporary market stalls. The intention behind having removable market stalls is to provide flexibility for the site as a whole so it can expand and contract to cater to specific events and festivals. As a result, the site would have the ability to transform from a highly dense market with little open public space to more of an open park atmosphere to allow for larger crowds during busy festival seasons. The idea of using temporary market stalls also affords the potential to generate income for the community if a small monetary figure was placed on the rental of these stalls for retail purposes. 


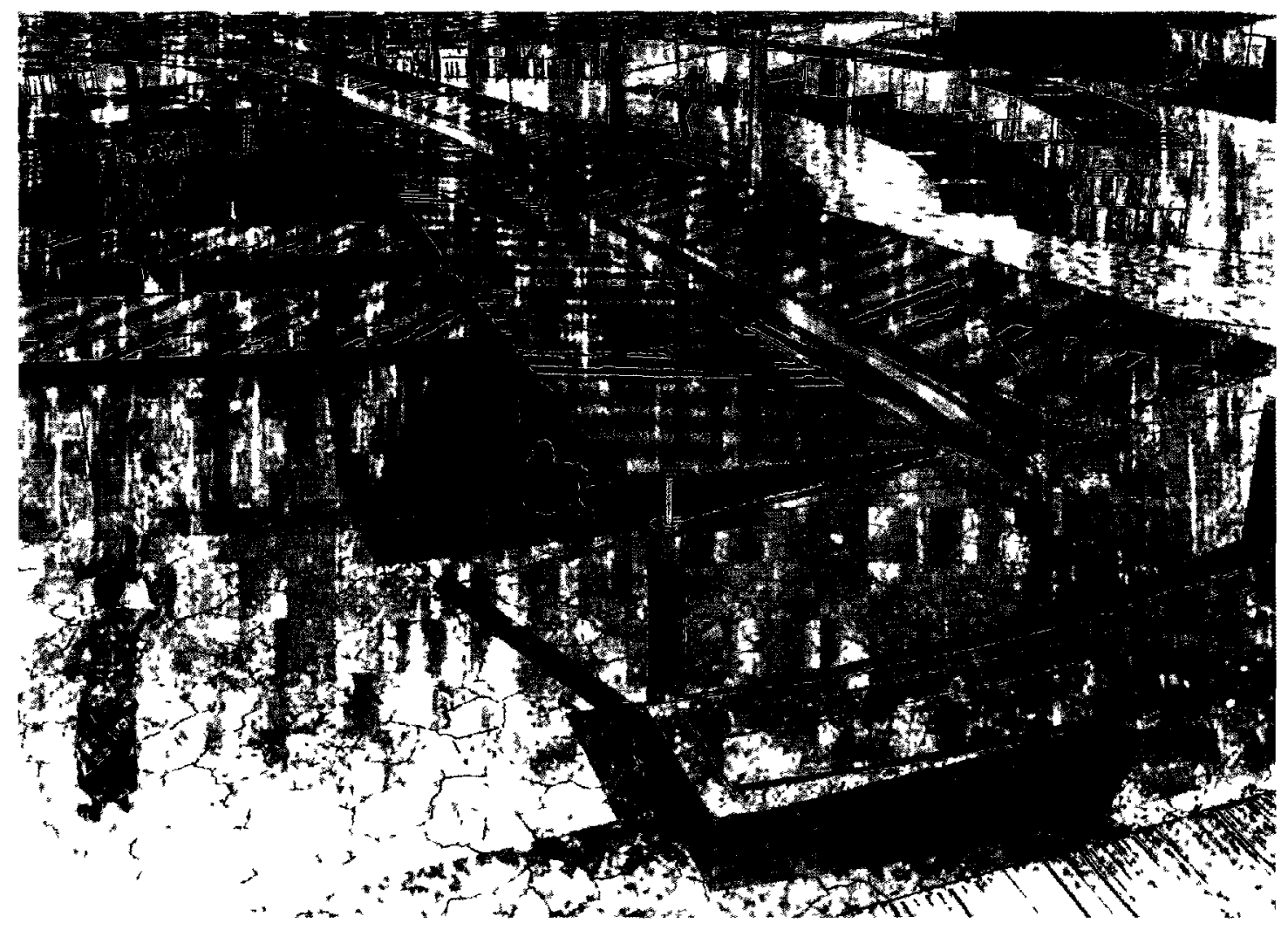

Fig. 7.21 - Cultural Market amphitheatre and water detail. Léogâne, Haiti.

The central area of the Cultural Market is where all other program radiates out from. It is the centerpiece of the market project and is where the amphitheatre is located. The intention of this large covered public facility is to provide a multi-purposed area for Léogâne where citizens can host a number of functions, including music festivals (such as Rara), arts festivals (through local and national arts organizations C.A.H.A \& H.P.P), be a venue for holding public forums and political speeches, offer casual recreational space for activities and sports, and generally be a safe, central gathering place in which to congregate. 

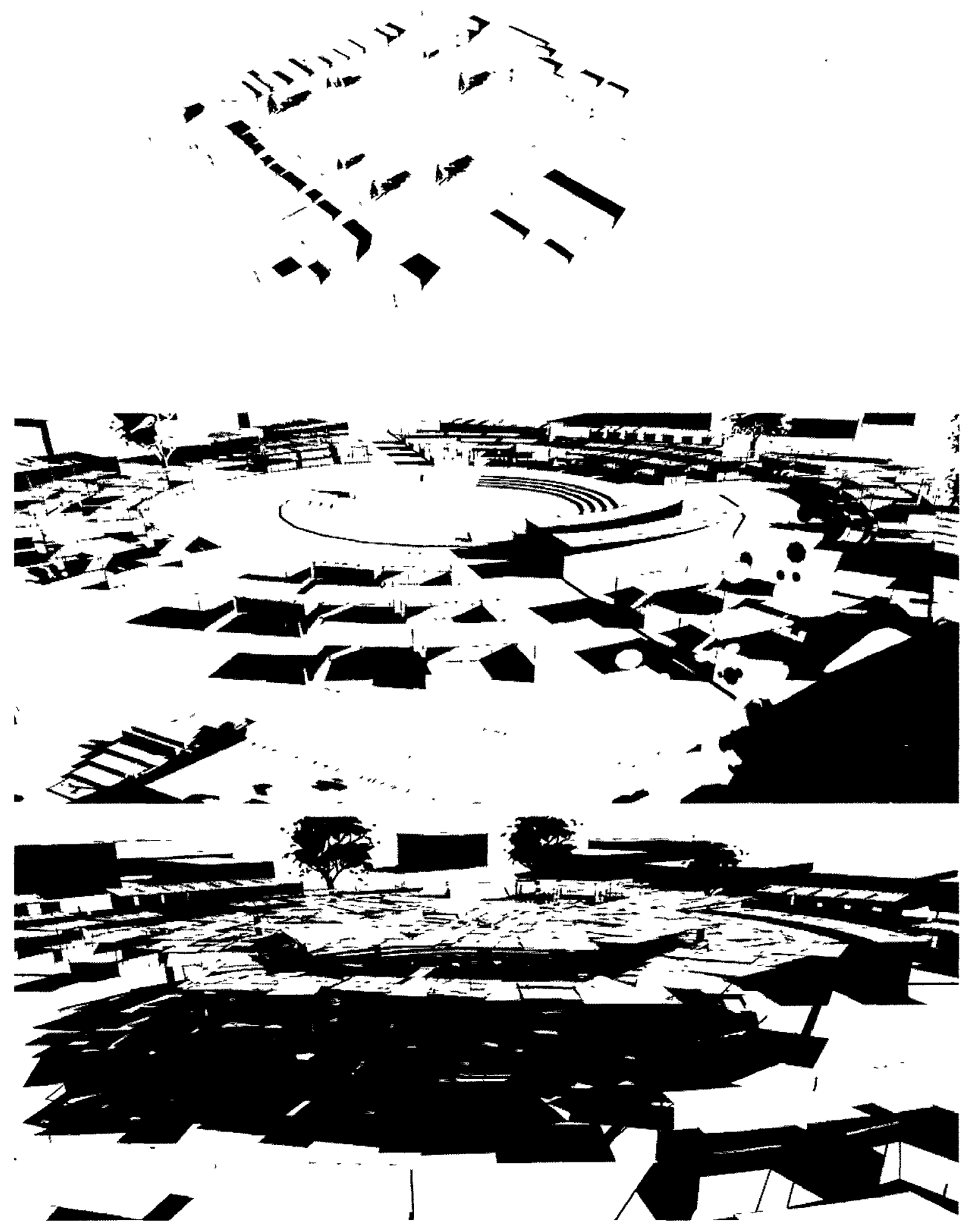

Fig. 7.22 - Cultural Market model documenting an accumulative progression from existing site conditions to the final project of architecture. 

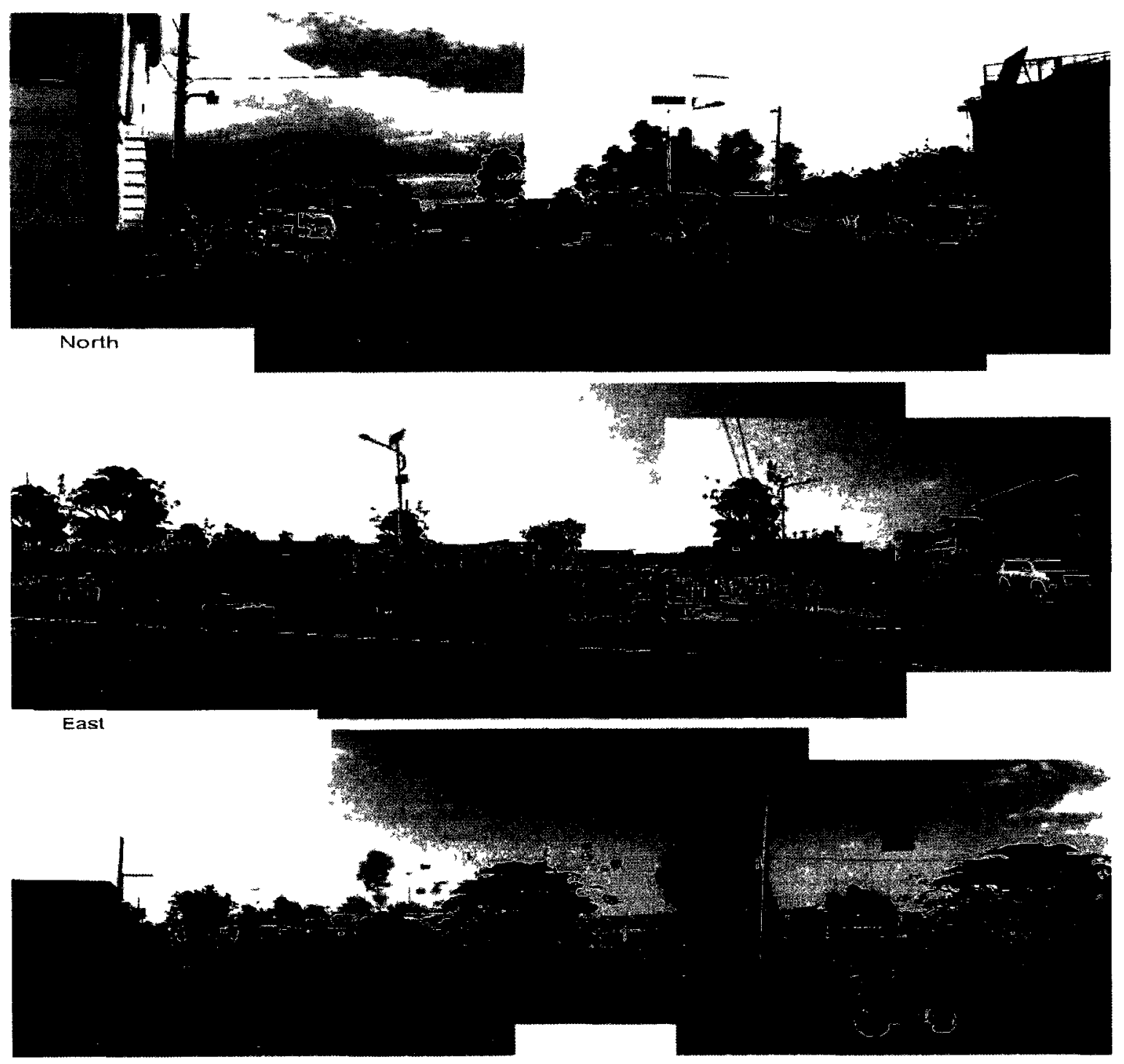

South

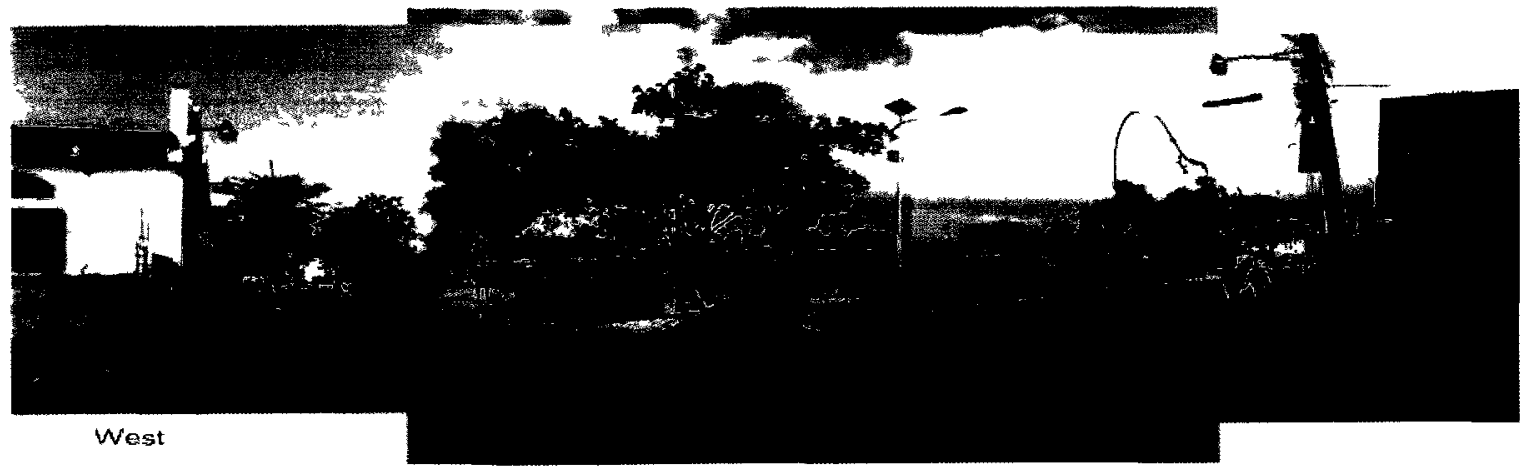

Fig. 7.23 - Existing site documentation panel with final model. Views of the site from each major intersection surrounding the Léogâne Cultural Market. 


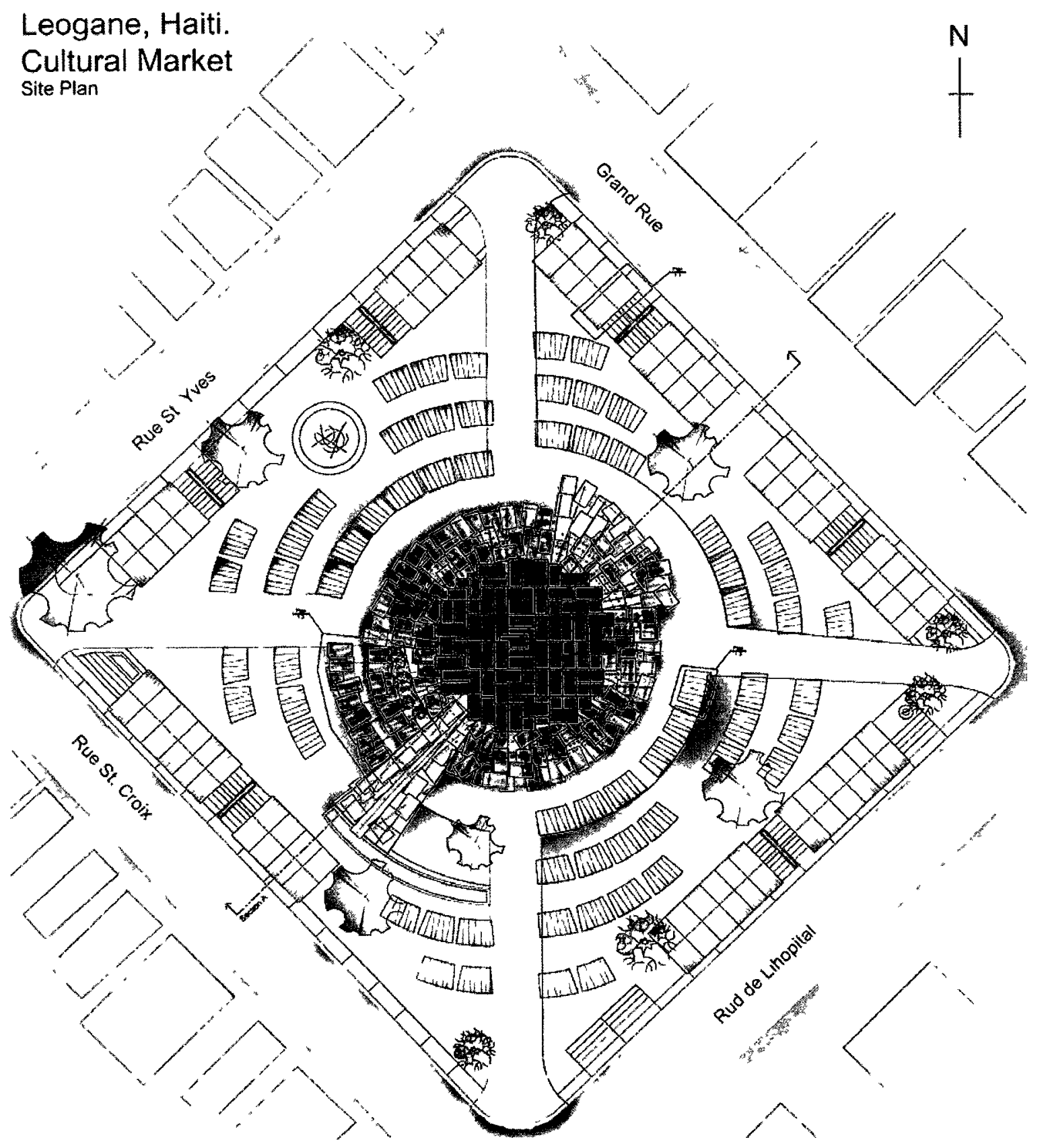

Fig. 7.24 - Cultural Market final site plan, Léogâne, Haiti. 


\section{Conclusion}

The design of towns, villages, community buildings, houses and various other types of architecture in a post-disaster situation is not a prescriptive science. This research paper clearly demonstrates, however, that if there hope of ever achieving long-term community subsistence, the inclusion of local knowledge and community collaboration are two fixed deliverables that must be considered.

Examining both successful and failed post-disaster global case studies has provided strong evidence that a project's long-term success is hinged on the involvement of local culture and project beneficiaries as key contributors. In Nick Wates' Community Planning Handbook he identifies these issues as important, however his work does not fully speak to their crucial significance within a developing nation context, as ascertained in the case studies examined.

The Flores Island, Indonesia earthquake and tsunami case study typifies what an unsuccessful rebuilding initiative entails. This quick-fix permanent resettlement was in direct opposition to everything the Wuring and Babi Island fishing communities stood for, with its primary focus instead on reducing vulnerability to future disasters. The rebuilding plan did not take into consideration crucial cultural and lifestyle predilections such as the mixed religious beliefs within the community, the need for specific community organizations, and most pointedly the importance of geography to living-and making a living -next to the sea. 
In contrast, the 2005 Pakistan and the 1999 Colombia earthquake rebuilding plans are precedents that reference best-case scenarios in organizing and implementing locally driven rebuilding initiatives. Both these rebuilding plans widely heeded specific community needs, incorporated the knowledge and training of beneficiaries, incorporated vernacular elements from the culture, and as a result had rebuilding projects that were accepted within their respective communities.

The Rebuilding Léogâne Design Workshop explored the potentials and limits of locally driven design collaboration taking place post-disaster within the framework of a developing nation. The workshop afforded a lens on the collective effort, in real-time, of community members and international participants as they worked toward a rebuilding planning process. The fact that this workshop was so well-attended by such engaged participants validated the importance all stakeholders placed on the process. This exercise also aligns with the direction taken by the Pakistan and Colombia case studies whereby the deliverables were community specific and based upon the needs and wants of the local people.

Based on preliminary research, and lessons learned from the Rebuilding Léogâne Design Workshop, a hypothetical project was proposed which analyzes the cultural resilience of the disaster-struck community of Léogâne. The proposed Cultural Market project identifies the local strength of the arts and music culture and addresses community concerns such as connectivity and vulnerability reduction. In essence, the Cultural Market embodies all gathered research and affirms the necessity for locally driven collaborative design for post- 
disaster rebuilding.

It has been established that designing culturally specific post-disaster architecture is a highly involved process. As discovered, no two post-disaster communities are the same, and since successful rebuilding requires locally driven information the concept of a generalized formula for disaster rebuilding can be dismissed. Evidence supports, however, that there is still a need for disaster response standardization within the developing world targeted to address long-term subsistence. As was observed firsthand at the Rebuilding Léogâne Design Workshop, progress had been impeded by too many individual organizations trying to orchestrate their own rebuilding agendas and not enough accumulative collaboration working toward an overall vision. Although much of the disorganization can be attributed to the unstable nature of developing countries, I believe there are too many small aid organizations with far too many short-term agendas complicating the process.

I feel that if this project were to be continued it would need to be validated through a built variation placed within the context of this post-disaster community. This research investigation would explore the intricacies of its locally inspired construction detailing. As someone from the profession of architecture, I would utilize my technical understandings of detailing and propose construction details inspired by and for Léogâne. In my research I reference Nick Wates and his Community Planning Handbook as a primary reference for anyone involved in the field of community planning. I would propose a similar document, but one more geared toward the project-specific construction detailing rooted within the 
readily available local materials. This document would visually illustrate structurally sound and innovative ways to construct within Haiti from an economical and efficiency standpoint. I would explore alternatives to basic details such as a down spout for drainage, bamboo connections, concrete block to bamboo connections, and alternative window and partition wall variations. In the materials section within the previous chapter I have included some sketches of various construction details that explore the potential of using local materials. The next step is to resolve these construction details and create a catalogue that could ideally be left within the project community to inspire and teach local people innovative ways to approach and rebuild their community.

As this paper has illustrated, there are many steps and considerations to achieving a successful post-disaster rebuilding initiative. One research-based solution is proposed in this paper, the Léogâne Cultural Market. This project has been substantiated through research and field investigation as a viable model that could be central to the rebuilding initiative. The intent of a community-led rebuild initiative such as this is to serve as a central hub to bolster cultural resiliency. Ultimately, however, the Cultural Market's true success can only be determined through use and acceptance by its end users, the citizens of Léogâne. 


\section{Figures}

1.1 Taq \& Dhajji construction techniques. From: Randolph, Langenbach. “Don't Tear It Down! Preserving the Earthquake Resistant Vernacular Architecture of Kashmir".p.19.

1.2 An example of Taq construction in Srinagar, India. From: Randolph, Langenbach. "Don't Tear It Down! Preserving the Earthquake Resistant Vernacular Architecture of Kashmir'.p.19.

1.3 Dhajji field demonstration, Pakistan. From: "Handbook for Dhajji Construction", <http://sheltercentre.org/ meeting/material/Dhajji+ Construction+and+Confined+masonry>.

1.4 Post 1992 Earthquake \& Tsunami at Wuhring, Flores Island Indonesia. From: "The Major Tsunamis of 1992", <http://www.smate.wwu.edu/ teched/geology/tsu-Nicalndo92.html>.

1.5 Earthquake \& Tsunami community relocation project in Nangahure, 1992. From: "Cultural Considerations for Post Disaster Reconstruction Post- Tsunami Challenges", <http://www.adpc.net/irc06/2005/4-6/TBindo1.pdf>.

1.6 A 'new fishing village' built by the Babi Island Fisherman on the shores of Nangahale, March 2001. From: "'Cultural Considerations for Post Disaster Reconstruction Post- Tsunami Challenges", <http://www.adpc.net/irc06/ 2005/4-6/TBindo1.pdf>.

2.1 Capacities and vulnerabilities analysis matrix. From: Anderson, Mary B et al. "Rising from the Ashes: Development Strategies in Times of Disaster".p.12.

2.2 Activity Categories/Actors chart from Columbia post-earthquake housing rebuilding initiative organized by the Coffee Growers Organization; Jan 25,1999. From: Davidson, C.H. et al. "Truths and Myths about Community Participation in Post-Disaster Housing Projects".p.104.

3.1 Nick Wates' Participation Matrix. From: Wates,Nick. "The Community Planning Handbook".p.10.

3.2 Participatory design prioritizing game, Rebuilding Léogâne Workshop: Haiti. Image by author.

4.1 Shaded relief map of Haiti. From: "University of Texas Libraries", <http://www.lib.utexas.edu/maps/haiti.html>. 
4.2 Earthquake intensity map from January 12, 2010 Haiti. From: "Haiti Earthquakes, Maps \& Data", <http://www.gelib.com/haiti-earthquake.htm>.

5.1 Town of Léogâne Haiti aerial photograph. From: "Google Earth Images", <http://maps.google.ca/>.

6.1 Map of Rara Bands within the Léogâne commune. Image by author.

6.2 Jean-Eddy Beauvoir, Pastel and Acrylic Painting. Image by author.

6.3 Jean-Eddy Beauvoir, Mixed Media Collage. Image by author.

6.4 Jean-Eddy Beauvoir, Mixed Media Collage II. Image by author.

6.5 Ferdinand Madoche, Acrylic Painting. Image by author.

6.6 Kenson Desire, Clay wall hanging art. Image by author.

6.7 Kenson Desire displaying some of his art pieces at the 'Rebuilding Léogâne' Design Workshop March 17 2011. Image by author.

7.1 Léogâne Capacities \& Vulnerabilities diagram. Image by author.

7.2 Frame of reference site photos. From: "Google Earth Images", $<$ http://maps.google.ca/>.

7.3 Historic colonial public building March 2011, Léogâne Haiti. Image by author.

7.4 Historic old city hall building March 2011, Léogâne Haiti. Image by author.

7.5 Aerial photograph of the project site. From: "Google Earth Images", $<$ http://maps.google.ca/>.

7.6 Existing site documentation panel. Views of the site conditions from each major intersection surrounding the town square site. Image by author.

7.7 Sugarcane rum distillery, downtown Léogâne, Haiti. Image by author.

7.8 'Market Woman': Local market in Léogâne where charcoal is being sold. Image by author. 
7.9 Dried banana leaf partition wall panel, Léogâne, Haiti 2011. Image by author.

7.10 Concrete blocks stacked at a local construction site in Léogâne, Haiti 2011. Image by author.

7.11 Local material connection. Bamboo uprights on a small cooking shelter are fastened together with a dried banana leaf twine. Léogâne, Haiti 2011. Image by author.

7.12 Makeshift eaves trough detail. Léogâne, Haiti 2011. Image by author.

7.13 Bamboo and metal roofing construction detail. This model is a locally inspired connection that investigates a tectonic option for the temporary market stalls as part of this proposed project of architecture: Léogâne Cultural Market. Image by author.

7.14 Bamboo construction detail. Another locally inspired connection as a proposed strategy for the temporary market stalls. Image by author.

7.15 Bamboo construction detail sketch. Exploring potential structural connections for both temporary and permanent construction projects. Image by author.

7.16 Concrete block to bamboo connection. This sketch investigates an alternative method to provide lightweight structural roofing for small scale construction projects. Image by author.

7.17 Concrete block to bamboo connection II. This sketch explores bamboo as a form of partition wall and structural component extending its self from a concrete block wall. Image by author.

7.18 Cultural Market Master Plan. Depicting full occupancy of temporary market stalls located in circular formation radiating out from the central amphitheatre. Léogâne, Haiti. Image by author.

7.19 Cultural Market Master Plan II. Depicting partial occupancy of temporary market stalls allowing for more public space making room for festivals and events. Léogâne, Haiti. Image by author.

7.20 Cultural Market permanent artist stalls and festival bleachers. Léogâne, Haiti. Image by author.

7.21 Cultural Market amphitheatre and water detail. Léogâne, Haiti. Image by author. 
7.22 Cultural Market model documenting an accumulative progression from existing site conditions to the final project of architecture. Image by author.

7.23 Existing site documentation panel with final model. Views of the site from each major intersection surrounding the Léogâne Cultural Market. Image by author.

7.24 Cultural Market final site plan, Léogâne, Haiti. Image by author. 


\section{Bibliography}

Desire, Kenson. Interview by James Robinson \& 'Rebuilding Leogane Workshop', Culture \& Human Capital Workshop Group. Personal interview. 16 Mar. 2011. Leogane 'Rebuilding Workshop', Culture Workshop Group, Leogane Haiti. Interview by Culture workshop group Notre Dame research facility. Personal interview. 17 Mar. 2011.

Leogane 'Rebuilding Workshop', Education Workshop Group., Leogane Haiti. Interview by Education workshop group Notre Dame research facility. Personal interview. 17 Mar. 2011.

Leogane 'Rebuilding Workshop', Healthcare Workshop Group., Leogane Haiti. Interview by Healthcare workshop group Notre Dame research facility. Personal interview. 17 Mar. 2011.

Leogane 'Rebuilding Workshop', Housing Workshop Group., Leogane Haiti. Interview by Housing workshop group Notre Dame research facility. Personal interview. 17 Mar. 2011.

Amplifying the Diminished Voice. Dir. Sheri Blake. Perf. Detriot Design Centre Participants. Detroit Collarorative Design Center, 2006. DVD.

Anderson, Mary B., and Peter J. Woodrow. Rising from the Ashes: Development Strategies in Times of Disaster. London: ITDG Publishing, 1998. Print.

Boeni, Teddy, and Rohit Jigyasuii. Cultural Considerations for Post Disaster Reconstruction Post-Tsunami Challenges. Online: Google Scholar essay, 2004. Print.

CMHC,SBC,Minto. "Designing for Post-Disaster Reconstruction Charette: A Canadian Response Report." Designing for Post-Disaster Reconstruction. N.p., n.d. Web. 2 Oct. 2010. <www.sbcanada.org/online_pdf/Charrettes/sbc-reconstructioncharrette.pdf>.

Davidson, C.H., C. Johnson, G. Lizarralde, N. Dikmen, and A. Sliwinski. "Truths and myths about community participation in post-disaster housing projects." Habitat International 31.1 (2007): 100-115. Print.

Ellen, Roy. Indigenous Environmental Knowledge and its Transformations: Critical Anthropological Perspectives (Studies in Environmental Anthropology). 1 ed. New York: Routledge, 2000. Print. 
Federal Research Division, Library of Congress. "Haiti Country Profile.". N.p., 1 May 2006. Web. 1 Mar. 2011. <http://www.Icweb2.loc.gov/frd/cs/profiles/Haiti.pdf>.

Federal Research Division, Library of Congress. "Haiti Country Profile.". N.p., 1 Dec 1989. Web. 1 Mar. 2011. <http://www.lcweb2.loc.gov/cgi-bin/query/r?frd/ cstdy: @ field\%28DOCID+ht0064\%29>.

Gosling, Nicholas. "Helping Haiti | School Construction News." School Construction News. N.p., 10 Aug. 2010. Web. 25 Sept. 2010.

<http://www.schoolconstructionnews.com/articles/2010/08/10/architectsplanners-join-rebuild-haiti-s-schools>.

Government, Haiti. "Action Plan for National Recovery and Development of Haiti." Government of the Republic of Haiti 1.1 (2010): 1-55. Print.

"Haiti Earthquake Maps and Data Â« Google Earth Library." Google Earth Library. N.p., n.d. Web. 24 Feb. 2011. <http://www.gelib.com/haiti-earthquake.htm>.

"Haiti Maps - Perry-CastaÃ \pm da Map Collection - UT Library Online." University of Texas Libraries. N.p., n.d. Web. 20 Feb. 2011.

<http://www.lib.utexas.edu/maps/haiti.html>.

"Haiti | Schools for Children." Schools for Children | Home. N.p., 22 Oct. 2010. Web. 27 Sept. 2010. <http://www.schoolsforchildren.org/?page_id=352>.

Heath, Kingston Wm.. Vernacular Architecture and Regional Design: Cultural Process and Environmental Response. London: Architectural Press, 2009. Print.

Hester, Randolph T.. Community design primer . Mendocino, Calif.: Ridge Times Press, 1990. Print.

Langenbach, Randolph. Don't Tear It Down! Preserving the Earthquake Resistant Vernacular Architecture of Kashmir. 1 ed. Oakland: Oinfroin Media, 2009. Print.

McAlister, Elizabeth A.. Rara!: vodou, power, and performance in Haiti and its diaspora. Berkeley: University of California Press, 2002. Print.

Ockwell, David, and Yvonne Rydin. "Conflicting Discourses of Knowledge." Environmental Ploitics 15.3 (2006): 379-398. Print.

Oliver-Smith, A. "Post-Disaster Housing Reconstruction and Social Inequality: A Challenge to Policy and Practice." Disasters 14 (1990): 7-19. Print.

Oliver-Smith, Anthony. "Successes and Failures in Post-Disaster Resettlement." Disasters 15.1 (2007): 12-23. Print.

Sandercock, Leonie, Giovanni Attili, and Sheri Blake. "Participatory Design and Howard 
Roark: The Story of the Detroit Collaborative Design Center." Multimedia explorations in urban policy and planning beyond the flatlands. Dordrecht : Springer, 2010. 225-243. Print.

Sanoff, Henry. Community participation methods in design and planning . New York: Wiley, 2000. Print.

Schacher, Tom. "Dhajji Construction and Confined Masonry | Shelter Centre." Shelter Centre | the NGO supporting the humanitarian community in post-conflict and disaster shelter and housing. N.p., n.d. Web. 23 Sept. 2010.

<http://www.sheltercentre.org/meeting/material/Dhajji+Construction+and+Confin ed+Masonry>.

"UN-HABITAT |EarthQuake Disaster - Pakistan ." UN-HABITAT - United Nations Human Settlements Programme. N.p., n.d. Web. 23 Sept. 2010.

<http://ww2.unhabitat.org/earthquake/Pakistan_earthquake.asp>.

United Nations Development Programme. United Nations Human Development Report 2010. United Kingdom: Palgrave Macmillan, 2010. Print.

University, Notre Dame. "Rebuilding Leogane Planning Workshop." University of Notre Dame. N.p., 1 Jan. 2011. Web. 1 Feb. 2011. <http://www.nd.edu/ ce4hait//index.htm>.

Ward, Peter. Self-Help Housing: A Critique. New York: Hw Wilson Co, 1982. Print.

Wates, Nick. The Community Planning Handbook: How People Can Shape Their Cities, Towns and Villages in Any Part of the World. London: Earthscan Publications Ltd., 2000. Print.

Zehr, Mary Ann. "Education Week: Schools Mobilize for Haiti's Earthquake Recovery." Education Week American Education News Site of Record. N.p., 14 Jan. 2010. Web. 27 Sept. 2010. <http://www.edweek.org/ew/articles/2010/01/14/18haiti.h29.html?qs=haiti>. 